\title{
Tissue-Specific Alteration of Metabolic Pathways Influences Glycemic
}

\section{Regulation}

Natasha H. J. $\mathrm{Ng}^{1,2 *}$, Sara M. Willems ${ }^{3 *}$, Juan Fernandez ${ }^{4}$, Rebecca S. Fine ${ }^{5,6,7}$, Eleanor Wheeler $^{8,3}$, Jennifer Wessel ${ }^{9,10}$, Hidetoshi Kitajima ${ }^{4}$, Gaelle Marenne ${ }^{8}$, Jana K. Rundle ${ }^{1}$, Xueling $\mathrm{Sim}^{11,12}$, Hanieh Yeghootkar ${ }^{13}$, Nicola L. Beer ${ }^{1}$, Anne Raimondo ${ }^{1}$, Andrei I. Tarasov ${ }^{1}$, Soren K. Thomsen $^{1,14}$, Martijn van de Bunt ${ }^{4,1}$, Shuai Wang ${ }^{15}$, Sai Chen ${ }^{12}$, Yuning Chen ${ }^{15}$, Yii-Der Ida Chen $^{16}$, Hugoline G. de Haan ${ }^{17}$, Niels Grarup ${ }^{18}$, Ruifang Li-Gao ${ }^{17}$, Tibor V. Varga ${ }^{19}$, Jennifer L Asimit $^{8,20}$, Shuang Feng ${ }^{21}$, Rona J. Strawbridge ${ }^{22,23}$, Erica L. Kleinbrink ${ }^{24}$, Tarunveer S. Ahluwalia $^{25,26}$, Ping An ${ }^{27}$, Emil V. Appel ${ }^{18}$, Dan E Arking ${ }^{28}$, Juha Auvinen ${ }^{29,30}$, Lawrence F. Bielak $^{31}$, Nathan A. Bihlmeyer ${ }^{28}$, Jette Bork-Jensen ${ }^{18}$, Jennifer A. Brody ${ }^{32,33}$, Archie Campbell ${ }^{34}$, Audrey Y Chu ${ }^{35}$, Gail Davies ${ }^{36,37}$, Ayse Demirkan ${ }^{38}$, James S. Floyd ${ }^{32,33}$, Franco Giulianini ${ }^{35}$, Xiuqing Guo ${ }^{16}$, Stefan Gustafsson ${ }^{39}$, Benoit Hastoy ${ }^{1}$, Anne U. Jackson ${ }^{12}$, Johanna Jakobsdottir ${ }^{40}$, Marjo-Riitta Jarvelin ${ }^{41,29,42}$, Richard A. Jensen ${ }^{32,33}$, Stavroula Kanoni ${ }^{43}$, Sirkka KeinanenKiukaanniemi ${ }^{44,45}$, Jin $\mathrm{Li}^{46}$, Man $\mathrm{Li}^{47,48}$, Kurt Lohman ${ }^{49}$, Yingchang $\mathrm{Lu}^{50,51}$, Jian'an Luan ${ }^{3}$, Alisa K. Manning ${ }^{52,53}$, Jonathan Marten ${ }^{54}$, Carola Marzi ${ }^{55,56}$, Karina Meidtner ${ }^{57,56}$, Dennis O. MookKanamori $^{17,58}$, Taulant Muka ${ }^{59,38}$, Giorgio Pistis ${ }^{60,61}$, Bram Prins ${ }^{8}$, Kenneth M. Rice ${ }^{32,62}$, Neil Robertson $^{4}$, Serena Sanna ${ }^{60,63}$, Yuan Shi ${ }^{64}$, Albert Vernon Smith ${ }^{40,65}$, Jennifer A. Smith ${ }^{31,66}$, Lorraine Southam $^{8,4,67}$, Heather M. Stringham ${ }^{12}$, Salman M. Tajuddin ${ }^{68}$, Vinicius Tragante ${ }^{69}$, Sander W. van der Laan $^{70,71}$, Helen R. Warren ${ }^{43,72}$, Jie Yao ${ }^{16}$, Andrianos M. Yiorkas ${ }^{73,74}$, Weihua Zhang $^{75,76}$, Wei Zhao ${ }^{31}$, Emma Ahlqvist ${ }^{77}$, Mariaelisa Graff ${ }^{78}$, Heather M. Highland ${ }^{78,79}$, Anne E. Justice $^{78}$, Ken Sin Lo ${ }^{80}$, Eirini Marouli ${ }^{43}$, Carolina Medina-Gomez ${ }^{38,81}$, Saima Afaq ${ }^{41}$, Wesam A Alhejily $^{43,82}$, Najaf Amin ${ }^{38}$, Folkert W. Asselbergs ${ }^{69,83,84}$, Lori L. Bonnycastle ${ }^{85}$, Michiel L. Bots ${ }^{86}$, Ivan Brandslund ${ }^{87}$, Ji Chen $^{8}$, Cramer Christensen ${ }^{88}$, John Danesh ${ }^{89}$, Renée de Mutsert ${ }^{17}$, Abbas Dehghan $^{38,90,91}$, Tapani Ebeling ${ }^{92}$, Paul Elliott ${ }^{41,93,94}$, EPIC-InterAct Consortium, Aliki-Eleni Farmaki $^{95,96}$, Jessica D. Faul ${ }^{66}$, Paul W. Franks ${ }^{19,97,6}$, Steve Franks ${ }^{98}$, Andreas Fritsche ${ }^{99,56}$, Anette P. Gjesing ${ }^{18}$, Mark O. Goodarzi ${ }^{100}$, Vilmundur Gudnason ${ }^{40,65}$, Göran Hallmans ${ }^{101}$, Tamara B. Harris ${ }^{40}$, Karl-Heinz Herzig ${ }^{102,103}$, Marie-France Hivert ${ }^{104,105}$, Jan-Håkan Jansson ${ }^{106}$, Min A Jhun $^{31,107}$, Torben Jørgensen ${ }^{108,109,110}$, Marit E. Jørgensen ${ }^{26,111}$, Pekka Jousilahti ${ }^{112}$, Eero Kajantie $^{113,112,114,115}$, Maria Karaleftheri ${ }^{116}$, Sharon L.R. Kardia ${ }^{31}$, Leena Kinnunen ${ }^{112}$, Heikki A. Koistinen ${ }^{112,117,118}$, Pirjo Komulainen ${ }^{119}$, Peter Kovacs ${ }^{120,121}$, Johanna Kuusisto ${ }^{122}$, Markku Laakso $^{122}$, Leslie A. Lange ${ }^{123}$, Lenore $\mathrm{J}^{112}$. Launer ${ }^{40}$, Jung-Jin Lee ${ }^{124}$, Aaron Leong ${ }^{125}$, Jaana Lindström $^{112}$, Jocelyn E. Manning Fox ${ }^{126,127}$, Satu Männistö ${ }^{112}$, Nisa M Maruthur ${ }^{128,129,48}$, Leena Moilanen ${ }^{130}$, Antonella Mulas ${ }^{60,131}$, Mike A. Nalls ${ }^{132,133}$, Matthew Neville ${ }^{1}$, James S. Pankow $^{134}$, Alison Pattie ${ }^{37}$, Eva R.B. Petersen ${ }^{135}$, Hannu Puolijoki ${ }^{136}$, Asif Rasheed ${ }^{137}$, Paul Redmond $^{37}$, Frida Renström ${ }^{19,101}$, Michael Roden ${ }^{138,139,56}$, Danish Saleheen ${ }^{124,137}$, Juha Saltevo ${ }^{140}$, Kai Savonen ${ }^{119,141}$, Sylvain Sebert ${ }^{44,42}$, Tea Skaaby ${ }^{108}$, Kerrin S Small ${ }^{142}$, Alena Stančáková ${ }^{122}$, Jakob Stokholm ${ }^{25}$, Konstantin Strauch ${ }^{143}$, E-Shyong Tai ${ }^{144,11,145}$, Kent D. Taylor $^{16}$, Betina H. Thuesen ${ }^{108}$, Anke Tönjes ${ }^{121}$, Emmanouil Tsafantakis ${ }^{146}$, Tiinamaija Tuomi $^{147,148,149}$, Jaakko Tuomilehto ${ }^{112,150,151,152}$, Understanding Society Scientific Group, Matti Uusitupa $^{153}$, Marja Vääräsmäki ${ }^{154,155}$, Ilonca Vaartjes ${ }^{86}$, Magdalena Zoledziewska ${ }^{60}$, Goncalo Abecasis $^{61}$, Beverley Balkau ${ }^{156}$, Hans Bisgaard ${ }^{25}$, Alexandra I. Blakemore ${ }^{74,73}$, Matthias Blüher $^{120,121}$, Heiner Boeing ${ }^{157}$, Eric Boerwinkle ${ }^{158}$, Klaus Bønnelykke ${ }^{25}$, Erwin P. Bottinger ${ }^{50}$, 
Mark J. Caulfield ${ }^{43,72}$, John C Chambers ${ }^{41,76,159}$, Daniel I Chasman ${ }^{35,160,161}$, Ching-Yu Cheng ${ }^{64,162,163}$, Anne Clark ${ }^{1}$, Francis S. Collins ${ }^{85}$, Josef Coresh ${ }^{48,129}$, Francesco Cucca ${ }^{60,131}$, Gert J. de Borst ${ }^{164}$, lan J. Deary ${ }^{36,37}$, George Dedoussis ${ }^{95}$, Panos Deloukas ${ }^{43,165}$, Hester M. den Ruijter $^{70}$, Josée Dupuis ${ }^{15,166}$, Michele K. Evans ${ }^{68}$, Ele Ferrannini ${ }^{167}$, Oscar H Franco ${ }^{38,59}$, Harald Grallert $^{55,56}$, Leif Groop ${ }^{168,149}$, Torben Hansen ${ }^{18,169}$, Andrew T. Hattersley ${ }^{170}$, Caroline Hayward $^{54}$, Joel N. Hirschhorn ${ }^{171,6,7}$, Arfan Ikram ${ }^{38}$, Erik Ingelsson ${ }^{46,39,172,173}$, Fredrik Karpe ${ }^{1,174}$, Kay-Tee Kaw ${ }^{175}$, Wieland Kiess ${ }^{176}$, Jaspal S Kooner ${ }^{177,76,159}$, Antje Körner ${ }^{176}$, Timo Lakka ${ }^{178,119,141}$, Claudia Langenberg ${ }^{3}$, Lars Lind ${ }^{179}$, Cecilia M Lindgren ${ }^{4,180}$, Allan Linneberg ${ }^{108,181}$, Leonard Lipovich $^{24,182}$, Ching-Ti Liu ${ }^{15}$, Jun Liu ${ }^{38}$, Yongmei Liu ${ }^{183}$, Ruth J.F. Loos ${ }^{50,184}$, Patrick E. MacDonald ${ }^{126,127}$, Karen L. Mohlke ${ }^{185}$, Andrew D Morris ${ }^{186}$, Patricia B. Munroe ${ }^{43,72}$, Alison Murray $^{187}$, Sandosh Padmanabhan ${ }^{188}$, Colin N A Palmer ${ }^{189}$, Gerard Pasterkamp ${ }^{70,71}$, Oluf Pedersen $^{18}$, Patricia A. Peyser ${ }^{31}$, Ozren Polasek ${ }^{190}$, David Porteous ${ }^{34}$, Michael A. Province ${ }^{27}$, Bruce M Psaty 32,33,191,192, Rainer Rauramaa ${ }^{119}$, Paul M Ridker ${ }^{35,160,193}$, Olov Rolandsson ${ }^{194}$, Patrik Rorsman ${ }^{1,174}$, Frits R. Rosendaal ${ }^{17}$, Igor Rudan $^{186}$, Veikko Salomaa ${ }^{112}$, Matthias B. Schulze ${ }^{57,56}$, Robert Sladek ${ }^{195,196}$, Blair H Smith ${ }^{189}$, Timothy D Spector ${ }^{142}$, John M. Starr ${ }^{197,36}$, Michael Stumvoll ${ }^{121}$, Cornelia M van Duijn ${ }^{38}$, Mark Walker ${ }^{198}$, Nick J. Wareham ${ }^{3}$, David R. Weir $^{66}$, James G. Wilson ${ }^{199}$, Tien Yin Wong ${ }^{64,162,163}$, Eleftheria Zeggini ${ }^{8,67}$, Alan B. Zonderman ${ }^{68}$, Jerome I. Rotter ${ }^{16}$, Andrew P. Morris ${ }^{200,4}$, Michael Boehnke ${ }^{12}$, Jose Florez ${ }^{201,202}$, Mark I McCarthy ${ }^{4,1,174}$, James B Meigs ${ }^{125,202}$, Anubha Mahajan ${ }^{4}$, Robert A. Scott ${ }^{3}$, Anna L Gloyn ${ }^{1,4,17 *^{+}}{ }^{\text {, }}$ Inês Barroso $0^{8,3} *^{+}$

*Authors contributed equally

${ }^{+}$Corresponding authors:

Anna L Gloyn - anna.gloyn@drl.ox.ac.uk

Inês Barroso- ines.barroso@mrc-epid.cam.ac.uk

1. Oxford Centre for Diabetes, Endocrinology and Metabolism, University of Oxford, Oxford, UK, OX3 7LE.

2. Stem Cells and Diabetes Laboratory, Institute of Molecular and Cell Biology, Agency for Science, Technology and Research (A*STAR), Singapore 138673, Singapore

3. MRC Epidemiology Unit, University of Cambridge School of Clinical Medicine, Institute of Metabolic Science, Cambridge Biomedical Campus, Cambridge, CB2 0QQ, UK.

4. Wellcome Centre for Human Genetics, University of Oxford, Oxford, UK, OX3 7BN

5. Department of Genetics, Harvard Medical School, Boston, MA, 02115

6. Division of Endocrinology and Center for Basic and Translational Obesity Research, Boston Children's Hospital, Boston, MA 02115

7. Broad Institute of MIT and Harvard, Cambridge, MA, 02142

8. Department of Human Genetics, Wellcome Sanger Institute, Genome Campus, Hinxton, Cambridge, CB10 1SA, UK

9. Departments of Epidemiology \& Medicine, Schools of Public Health \& Medicine, Indiana University, Indiaiapolis, IN 46202

10. Diabetes Translational Research Center, Indiana University School of Medicine, Indianapolis, IN 46202 
11. Saw Swee Hock School of Public Health, National University Health System, National University of Singapore, Singapore 117549, Singapore.

12. Department of Biostatistics and Center for Statistical Genetics, University of Michigan, Ann Arbor, MI 48109, USA

13. Genetics of Complex Traits, University of Exeter Medical School, University of Exeter, Exeter, EX2 5DW, UK

14. Present address: Vertex Pharmaceuticals Europe Ltd, Milton Park, Abingdon, OX14 4RW, UK

15. Department of Biostatistics, Boston University School of Public Health, Boston, MA, USA.

16. Institute for Translational Genomics and Population Sciences, Departments of Pediatrics and Medicine, LABiomed at Harbor-UCLA Medical Center, Torrance, CA, USA

17. Department of Clinical Epidemiology, Leiden University Medical Center, Leiden, 2333 ZA, the Netherlands

18. Novo Nordisk Foundation Center for Basic Metabolic Research, Faculty of Health and Medical Sciences, University of Copenhagen, 2200 Copenhagen, Denmark

19. Department of Clinical Sciences, Genetic and Molecular Epidemiology Unit, Lund University, Malmö, SE-205 02, Sweden

20. MRC Biostatistics Unit, Cambridge Biomedical Campus, Cambridge Institute of Public Health, Forvie Site, Robinson Way, Cambridge CB2 OSR, UK.

21. Department of Biostatistics, University of Michigan School of Public Health, Ann Arbor, Michigan, USA

22. UKRI Innovation Fellow at HDR UK,Mental Health and Wellbeing, Institute of Health and Wellbeing, College of Medical, Veterinary and Life Sciences, University of Glasgow, Room 113, 1 Lilybank Gardens ,Glasgow, G12 8RZ

23. Cardiovascular Medicine Unit, Department of Medicine Solna, Karolinska Institutet, Stockholm,171 76, Sweden

24. Center for Molecular Medicine and Genetics, Wayne State University, 540 E. Canfield St., 3208 Scott Hall, Detroit, Michigan 48201-1928, U.S.A.

25. COPSAC, Copenhagen Prospective Studies on Asthma in Childhood, Herlev and Gentofte Hospital 2820, University of Copenhagen, Copenhagen, Denmark

26. Steno Diabetes Center Copenhagen, 2820 Gentofte, Denmark

27. Department of Genetics, Division of Statistical Genomics, Washington University School of Medicine, St. Louis, Missouri 63108, USA

28. McKusick-Nathans Institute of Genetic Medicine, Johns Hopkins University School of Medicine, Baltimore, MD, USA.

29. Center for Life Course Health Research, University of Oulu, PO Box 8000, 90014 Oulu, Finland

30. Unit of Primary Care, Oulu University Hospital, Oulu, Finland

31. Department of Epidemiology, School of Public Health, University of Michigan, 1415 Washington Hgts, Ann Arbor, MI, 48109, USA

32. Cardiovascular Health Research Unit, University of Washington, 1730 Minor Ave. Suite 1360, Seattle, WA 98101, USA.

33. Department of Medicine, University of Washington, Seattle, WA, USA. 
34. Medical Genetics Section, Centre for Genomic and Experimental Medicine, Institute of Genetics and Molecular Medicine, University of Edinburgh, Edinburgh EH4 2XU, UK

35. Division of Preventive Medicine, Brigham and Women's Hospital, Boston MA, 02215, USA.

36. Centre for Cognitive Ageing and Cognitive Epidemiology, University of Edinburgh, Edinburgh EH8 9JZ

37. Department of Psychology, University of Edinburgh, Edinburgh, EH8 9JZ

38. Department of Epidemiology, Erasmus University Medical Center, Rotterdam, 3015 GE, The Netherlands.

39. Department of Medical Sciences, Molecular Epidemiology and Science for Life Laboratory, Uppsala University, Uppsala, 75237, Sweden

40. Icelandic Heart Association, Hotlasmari 1, 201 Kopavogur, Iceland.

41. Department of Epidemiology and Biostatistics, MRC-PHE Centre for Environment \& Health, School of Public Health, Imperial College London, London W2 1PG, United Kingdom.

42. Biocenter Oulu, University of Oulu, Oulu, Finland

43. William Harvey Research Institute, Barts and The London School of Medicine and Dentistry, Queen Mary University of London, UK.

44. Faculty of Medicine, Center for Life Course Health Research, University of Oulu, Oulu, Finland

45. MRC and Unit of Primary Care, Oulu University Hospital, Oulu, Finland

46. Department of Medicine, Division of Cardiovascular Medicine, Stanford University School of Medicine, Stanford, CA 94305, USA

47. Division of Nephrology, Internal Medicine, School of Medicine, University of Utah, Salt Lake City, Utah

48. Department of Epidemiology, Johns Hopkins Bloomberg School of Public Health, Baltimore, Maryland, USA.

49. Department of Biostatistical Sciences, Public Health Sciences, Wake Forest School of Medicine, Winston-Salem, NC 27157

50. The Charles Bronfman Institute for Personalized Medicine, The Icahn School of Medicine at Mount Sinai, New York, NY, 10069, USA.

51. Department of Medicine, Division of Epidemiology, Vanderbilt-Ingram Cancer Center, Vanderbilt Epidemiology Center, Vanderbilt University School of Medicine Nashville, TN 37203 USA

52. Center for Human Genetics Research, Massachusetts General Hospital, Boston, MA, 02114, USA

53. Department of Medicine, Harvard Medical School, Boston, MA, USA.

54. Medical Research Council Human Genetics Unit, Institute of Genetics and Molecular Medicine, University of Edinburgh, Edinburgh EH4 2XU, UK.

55. Institute of Epidemiology II, Research Unit of Molecular Epidemiology, Helmholtz Zentrum München, German

56. German Center for Diabetes Research (DZD), 85764 München-Neuherberg, Germany.

57. Department of Molecular Epidemiology, German Institute of Human Nutrition PotsdamRehbruecke (DIfE), 14558 Nuthetal, Germany. 
58. Department of Public Health and Primary Care, Leiden University Medical Center, Leiden, 2333 ZA, the Netherlands

59. Institute of Social and Preventive Medicine (ISPM), University of Bern, Bern, Switzerland

60. Italian National Research Council, Institute of Genetics and Biomedic Research, Cittadella Universitaria, Monserrato (CA) 09042, Italy

61. Center for Statistical Genetics, University of Michigan, 1415 Washington Heights, 48109 Ann Arbor, MI, USA

62. Department of Biostatistics, University of Washington, Seattle, WA, USA.

63. University of Groningen, University Medical Center Groningen, Department of Genetics, 9700 RB Groningen, the Netherlands.

64. Singapore Eye Research Institute, Singapore National Eye Centre, The Academia, 20 College Road, Singapore 169856.

65. Faculty of Medicine, University of Iceland, 101 Reykjavik, Iceland

66. Survey Research Center, Institute for Social Research, University of Michigan, 426 Thompson St, Ann Arbor, MI 48104

67. Institute of Translational Genomics, Helmholtz Zentrum München, German Research Center for Environmental Health, Neuherberg, Germany

68. Laboratory of Epidemiology and Population Sciences, National Institute on Aging, National Institutes of Health, Baltimore, MD 21224, USA.

69. Department of Cardiology, Division Heart \& Lungs, University Medical Center Utrecht, Utrecht University, 3584CX Utrecht, The Netherlands

70. Experimental Cardiology Laboratory, Division Heart and Lungs, University Medical Center Utrecht, 3584 CX Utrecht, Utrecht, the Netherlands

71. Laboratory of Clinical Chemistry and Hematology, University Medical Center Utrecht, 3584 CX Utrecht, Utrecht, the Netherlands

72. NIHR Barts Cardiovascular Research Unit, Barts and The London School of Medicine \& Dentistry, Queen Mary University, London, London, EC1M 6BQ, UK

73. Section of Investigative Medicine, Department of Medicine, Imperial College London, London, W12 ONN, UK

74. Department of Life Sciences, Brunel University London, London, UB8 3PH, UK

75. Department of Epidemiology and Biostatistics, Imperial College London, London W2 1PG, UK

76. Ealing Hospital, London North West Healthcare NHS Trust, Middlesex UB1 3HW, UK

77. Lund University Diabetes Centre, Department of Clinical Sciences Malmö, Lund University, Skåne University Hospital, SE-20502 Malmö, Sweden

78. Department of Epidemiology, University of North Carolina at Chapel Hill, Chapel Hill, NC, 27514, USA

79. Human Genetics Center, The University of Texas School of Public Health; The University of Texas Graduate School of Biomedical Sciences at Houston; The University of Texas Health Science Center at Houston, Houston, TX 77030, USA

80. Montreal Heart Institute, Université de Montréal, Montreal, Quebec, H1T 1C8, Canada

81. Department of Internal Medicine, Erasmus University Medical Center, Rotterdam, 3015 GE, The Netherlands

82. Department of Medicine, King Abdulaziz University, Jeddah, 21589, Saudi Arabia 
83. Durrer Center for Cardiovascular Research, Netherlands Heart Institute, 3501DG Utrecht, Netherlands

84. Institute of Cardiovascular Science, Faculty of Population Health Sciences, University College London, WC1E 6BT London, United Kingdom

85. Medical Genomics and Metabolic Genetics Branch, National Human Genome Research Institute, NIH, Bethesda, MD 20892, USA

86. Center for Circulatory Health, University Medical Center Utrecht, 3508GA Utrecht, the Netherlands

87. Department of Clinical Biochemistry, Lillebaelt Hospital Vejle, 7100 Vejle, Denmark

88. Medical Department, Lillebaelt Hospital Vejle, 7100 Vejle, Denmark

89. Department of Public Health and Primary Care, University of Cambridge, Cambridge, CB18RN, UK.

90. Department of Biostatistics and Epidemiology, MRC-PHE Centre for Environment and Health, School of Public Health, Imperial College London

91. UK Dementia Research Institute at Imperial College London, London, United Kingdom

92. Oulu University Hospital, 90220 Oulu, Finland

93. Imperial College NIHR Biomedical Research Centre, London, United Kingdom

94. Health Data Research UK - London at Imperial College London, United Kingdom

95. Department of Nutrition and Dietetics, School of Health Science and Education, Harokopio University, Athens, 17671, Greece

96. Department of Population Science and Experimental Medicine, Institute of Cardiovascular Science, University College London, London, UK

97. Department of Nutrition, Harvard School of Public Health, Boston, USA.

98. Institute of Reproductive and Developmental Biology, Imperial College London,W12 ONN London, UK

99. Department of Internal Medicine, Division of Endocrinology, Diabetology, Vascular Medicine, Nephrology, and Clinical Chemistry, University Hospital of Tübingen, Tübingen Germany.

100. Division of Endocrinology, Diabetes and Metabolism, Cedars-Sinai Medical Center, Los Angeles, CA, 90048, USA.

101. Department of Biobank Research, Umeå University, Umeå, SE-901 87, Sweden.

102. Institute of Biomedicine and Biocenter of Oulu, Faculty of Medicine, Medical Research Center Oulu and Oulu University Hospital (K.-H.H.), Oulu, Finland.

103. Department of Gastroenterology and Metabolism, Poznan University of Medical Sciences, 60-572 Poznan, Poland

104. Department of Population Medicine, Harvard Medical School, Harvard Pilgrim Health Care Institute, Boston, MA, USA

105. Diabetes Unit, Massachusetts General Hospital, Boston, MA, USA

106. Department of Public Health and Clinical Medicine, Umeå University, Umeå, SE-901 85, Sweden.

107. Division of Public Health Sciences, Fred Hutchison Cancer Research Center, Seattle, Washington, 98109, USA

108. Center for Clinical Research and Prevention, Bispebjerg and Frederiksberg Hospital, 2000 Frederiksberg, Denmark 
109. Department of Public Health, Faculty of Health and Medical Sciences, University of Copenhagen, 2200 Copenhagen, Denmark

110. Faculty of Medicine, University of Aalborg, 9100 Aalborg, Denmark

111. National Institute of Public Health, Southern Denmark University, 5000 Odense, Denmark

112. Department of Public Health Solutions, National Institute for Health and Welfare, FI00271 Helsinki, Finland

113. PEDEGO Research Unit, MRC Oulu, Oulu University Hospital and University of Oulu, Oulu, Finland

114. Norwegian University of Science and Technology, Department of Clinical and Molecular Medicine, Trondheim, Norway

115. Children's Hospital, Helsinki University Hospital and University of Helsinki

116. Echinos Medical Centre, Echinos, Greece.

117. University of Helsinki and Department of Medicine, Helsinki University Central Hospital, P.O.Box 340, Haartmaninkatu 4, Helsinki, FI-00029, Finland

118. Minerva Foundation Institute for Medical Research, Biomedicum $2 U$ Helsinki, Tukholmankatu 8, FI-00290 Helsinki, Finland

119. Foundation for Research in Health Exercise and Nutrition, Kuopio Research Institute of Exercise Medicine, Kuopio, 70100 Kuopio, Finland

120. Integrated Research and Treatment (IFB) Center AdiposityDiseases, University of Leipzig, Liebigstrasse 19-21, 04103 Leipzig, Germany

121. Department of Medicine, University of Leipzig, Liebigstrasse 18, 04103 Leipzig, Germany

122. Institute of Clinical Medicine, Internal Medicine, University of Eastern Finland, 70210 Kuopio, Finland

123. Department of Medicine, Division of Bioinformatics and Personalized Medicine, University of Colorado Denver, Aurora, CO, USA

124. Department of Biostatistics and Epidemiology, University of Pennsylvania, USA, 19104

125. Division of General Internal Medicine, Massachusetts General Hospital, Department of Medicine, Harvard Medical School, Boston, MA, USA

126. Alberta Diabetes Institute IsletCore, University of Alberta, Edmonton, Canada, T6G 2E1

127. Department of Pharmacology, University of Alberta, Edmonton, Canada, T6G 2E1

128. Department of Medicine, Division of General Internal Medicine, The Johns Hopkins University School of Medicine, Baltimore, Maryland

129. Welch Center for Prevention, Epidemiology, and Clinical Research, Johns Hopkins University, Baltimore, MD, USA.

130. Kuopio University Hospital, 70210 Kuopio, Finland

131. Dipartimento di Scienze Biomediche, Università degli Studi di Sassari, 07100 Sassari, Italy.

132. Laboratory of Neurogenetics, National Institute on Aging, Bethesda, MD, 20892, USA.

133. Data Tecnica International, LLC, Glen Echo, Maryland 20812, USA

134. Division of Epidemiology and Community Health, School of Public Health, University of Minnesota, Minneapolis, MN, 55455, USA.

135. Department of Clinical Biochemistry and Pharmacology, Odense University Hospital, 5000 Odense, Denmark 
136. South Ostobothnia Central Hospital, 60220 Seinajoki, Finland

137. Center for Non-Communicable Diseases, Karachi, Pakistan

138. Institute for Clinical Diabetology, German Diabetes Center, Leibniz Institute for Diabetes Research at Heinrich Heine University Düsseldorf, Düsseldorf, Germany

139. Division of Endocrinology and Diabetology, Medical Faculty, University Hospital Düsseldorf, Düsseldorf, Germany

140. Central Finland Central Hospital, 40620 Jyvaskyla, Finland

141. Department of Clinical Physiology and Nuclear Medicine, Kuopio University Hospital, 70029 Kuopio, Finland

142. Department of Twin Research and Genetic Epidemiology, King's College London, London, , SE1 7EH, UK

143. Institute of Genetic Epidemiology, Helmholtz Center Munich, German Research Center for Environmental Health, Neuherberg, Germany, German Center for Diabetes Research (DZD e.V.), Neuherberg, Germany

144. Department of Medicine, Yong Loo Lin School of Medicine, National University of Singapore, 1E Kent Ridge Road, Singapore 119228.

145. Duke-NUS Medical School, Singapore, 8 College Road, Singapore 169857

146. Anogia Medical Centre, Anogia, Greece.

147. Folkhälsan Research Centre, Helsinki, Finland

148. Department of Endocrinology, Helsinki University Central Hospital, Helsinki, Finland.

149. Institute for Molecular Medicine Finland FIMM, University of Helsinki, Helsinki, Finland

150. Department of Public Health, University of Helsinki, Helsinki, Finland

151. Department of Neuroscience and Preventive Medicine, Danube-University Krems, 3500 Krems, Austria

152. Saudi Diabetes Research Group, King Abdulaziz University, 21589 Jeddah, Saudi Arabia

153. Department of Public Health and Clinical Nutrition, University of Eastern Finland, 70210 Kuopio, Finland

154. PEDEGO Research Unit (Research Unit for Pediatrics, Dermatology, Clinical Genetics, Obstetrics and Gynecology), Medical Research Center Oulu (MRC Oulu), Oulu University Hospital and University of Oulu, Oulu, Finland.

155. Department of Welfare, Children, Adolescents and Families Unit, National Institute for Health and Welfare, Oulu, Finland

156. INSERM U1018, Centre de recherche en Épidémiologie et Santé des Populations (CESP), Villejuif, France

157. Department of Epidemiology, German Institute of Human Nutrition Potsdam-Rehbrücke (DIfE), 14558 Nuthetal, Germany.

158. The Human Genetics Center and Institute of Molecular Medicine, University of Texas Health Science Center, Houston, Texas, 77030, USA.

159. Imperial College Healthcare NHS Trust, London W12 OHS, UK

160. Harvard School of Medicine, Boston MA, 02115, USA

161. Division of Genetics, Brigham and Women's Hospital and Harvard Medical School, Boston MA, USA.

162. Ophthalmology \& Visual Sciences Academic Clinical Program (Eye ACP), Duke-NUS Medical School, Singapore, 8 College Road, Singapore 169857 
163. Department of Ophthalmology, Yong Loo Lin School of Medicine,National University of Singapore, Singapore, 1E Kent Ridge Rd, Singapore 119228.

164. Department of Vascular Surgery; Division of Surgical Specialties; University Medical Center Utrecht, 3584 CX Utrecht, the Netherlands

165. Princess Al-Jawhara Al-Brahim Centre of Excellence in Research of Hereditary Disorders (PACER-HD), King Abdulaziz University, Jeddah, Saudi Arabia

166. National Heart, Lung, and Blood Institute (NHLBI) Framingham Heart Study, Framingham, MA, USA.

167. CNR Institute of Clinical Physiology, Pisa, Italy, Department of Clinical \& Experimental Medicine, University of Pisa, Italy

168. Department of Clinical Sciences, Diabetes and Endocrinology, Lund University Diabetes Centre, Malmö, Sweden.

169. Faculty of Health Sciences, University of Southern Denmark, 5000 Odense, Denmark

170. University of Exeter Medical School, University of Exeter, Exeter, EX2 5DW, UK

171. Departments of Pediatrics and Genetics, Harvard Medical School, Boston, MA 02115

172. Stanford Cardiovascular Institute, Stanford University, Stanford, CA 94305

173. Stanford Diabetes Research Center, Stanford University, Stanford, CA 94305

174. Oxford NIHR Biomedical Research Centre, Churchill Hospital, Oxford, UK, OX3 7LE

175. Department of Public Health and Primary Care, Institute of Public Health, University of Cambridge, Strangeways Research Laboratory, Cambridge, CB1 8RN, UK

176. Pediatric Research Center, Department of Women \& Child Health, University of Leipzig, Leipzig, Germany

177. National Heart and Lung Institute, Imperial College London, London W12 ONN, UK

178. Institute of Biomedicine, School of Medicine, University of Eastern Finland, Kuopio Campus, 70211 Kuopio, Finland

179. Department of Medical Sciences, Molecular Epidemiology; EpiHealth, Uppsala University, Uppsala, 75185, Sweden

180. The Big Data Institute, Li Ka Shing Centre for Health Information and Discovery, University of Oxford, Oxford OX3 7BN, UK

181. Department of Clinical Medicine, Faculty of Health and Medical Sciences, University of Copenhagen, 2200 Copenhagen, Denmark

182. Department of Neurology, Wayne State University School of Medicine, Detroit, MI, USA

183. Department of Epidemiology \& Prevention, Division of Public Health Sciences, Wake Forest University, Winston-Salem, NC, 27157, USA.

184. The Mindich Child Health and Development Institute, The Icahn School of Medicine at Mount Sinai, New York, NY, 10069, USA.

185. Department of Genetics, University of North Carolina, Chapel Hill, NC, 27599, USA.

186. Usher Institute of Population Health Sciences and Informatics, University of Edinburgh, Edinburgh, EH16 4UX, UK

187. Aberdeen Biomedical Imaging Centre, University of Aberdeen, Foresterhill Health Campus, Aberdeen, AB25 2ZD

188. British Heart Foundation Glasgow Cardiovascular Research Centre, Institute of Cardiovascular and Medical Sciences, College of Medical, Veterinary and Life Sciences, University of Glasgow, Glasgow G12 8TA, UK 
189. Division of Population Health and Genomics, School of Medicine, University of Dundee, DUNEE DD2 4BF

190. Faculty of Medicine, University of Split, Split, Croatia

191. Departments of Epidemiology and Health Services, University of Washington, Seattle, WA, USA

192. Kaiser Permanent Washington Health Research Institute, Seattle, WA, USA

193. Division of Cardiovascular Medicine, Brigham and Women's Hospital, Boston MA 02115

194. Department of Public Health \& Clinical Medicine, Section for Family Medicine, Umeå University, Umeå, SE-901 85, Sweden

195. Department of Medicine, McGill University, Montreal (Quebec) Canada H4A 3J1

196. Department of Human Genetics, McGill University, Montreal (Quebec) Canada H3A 1B1

197. Alzheimer Scotland Dementia Research Centre, University of Edinburgh, Edinburgh, EH8 9JZ

198. Institute of Cellular Medicine, The Medical School, Newcastle University, Newcastle, NE2 $4 \mathrm{HH}, \mathrm{UK}$

199. Department of Physiology and Biophysics, University of Mississippi Medical Center, Jackson, MS, USA.

200. Department of Biostatistics, University of Liverpool, Liverpool L69 3GL, UK

201. Diabetes Unit, Department of Medicine, Massachusetts General, Boston, MA, USA.

202. Program in Medical Genetics, Broad Institute, Cambridge, MA, USA. 


\section{Tissue-Specific Alteration of Metabolic Pathways Influences Glycemic \\ 2 Regulation}

3

4 Highlights

5

\section{Summary}

- 23 novel coding variant associations (single-point and gene-based) for glycemic traits

- 51 effector transcripts highlighted different pathway/tissue signatures for each trait

- The exocrine pancreas and gut influence fasting and $2 \mathrm{~h}$ glucose, respectively

- Multiple variants in liver-enriched G6PC and islet-specific G6PC2 influence glycemia

Metabolic dysregulation in multiple tissues alters glucose homeostasis and influences risk for type 2 diabetes (T2D). To identify pathways and tissues influencing T2D-relevant glycemic traits (fasting glucose $[F G]$, fasting insulin $[\mathrm{FI}]$, two-hour glucose [2hGlu] and glycated hemoglobin [HbA1c]), we investigated associations of exome-array variants in up to 144,060 individuals without diabetes of multiple ancestries. Single-variant analyses identified novel associations at 21 coding variants in 18 novel loci, whilst gene-based tests revealed signals at two genes, TF ( $\mathrm{HbA1c}$ ) and G6PC (FG, FI). Pathway and tissue enrichment analyses of trait-associated transcripts confirmed the importance of liver and kidney for FI and pancreatic islets for $\mathrm{FG}$ regulation, implicated adipose tissue in $\mathrm{Fl}$ and the gut in $2 \mathrm{hGlu}$, and suggested a role for the non-endocrine pancreas in glucose homeostasis. Functional studies demonstrated that a novel FG/FI association at the liver-enriched G6PC transcript was driven by multiple rare loss-of-function variants. The FG/HbA1c-associated, islet-specific G6PC2 transcript also contained multiple rare functional variants, including two alleles within the same codon with divergent effects on glucose levels. Our findings highlight the value of integrating genomic and functional data to maximize biological inference. 


\section{Introduction}

It has long been recognized that rare and penetrant disease-causing mutations can pinpoint key proteins and pathways involved in human metabolism (Froguel et al., 1992; Gloyn et al., 2004; Montague et al., 1997). Type 2 diabetes (T2D) results from an inability of the pancreatic islet beta cells to produce and secrete sufficient insulin, compounded by the failure of metabolic tissues to respond to insulin and store glucose appropriately. Blood glucose levels are regulated by the co-ordination of homeostatic pathways operating across multiple tissues that control metabolism, therefore a clearer understanding of their relative roles is critical in guiding efforts to modulate them pharmacologically to treat T2D and prediabetes. In recent years, technological advances have made it possible to assay genetic variation genome-wide and at scale. These provide tremendous opportunities to understand metabolic differences within the physiological range through the study of quantitative fasting and post-challenge glycemic measures (Mahajan et al., 2015; Scott et al., 2012; Wessel et al., 2015; Wheeler et al., 2017a). These measures can influence the risk of developing pathophysiological conditions such as T2D and cardiovascular disease. However, as in all genome-wide association studies (GWAS), it has proven challenging to translate the associated genetic signals into biological pathways, as the vast majority of association signals lie within non-coding regions, and connecting them to their respective effector genes is less straightforward. There are to date over 97 loci reported to be associated with glycemic traits, across different genetic approaches (Wheeler et al., 2017b). One approach to facilitate identification of likely causal variants and transcripts is to focus on coding variation, whose effects on protein sequence can be predicted and functionally tested, facilitating identification of likely causal genes and the ensuing biological insights. This strategy has been successfully used to establish not only the effector genes but also the direction of effect of T2D risk alleles on protein function such as in the case of SLC30A8 (Flannick et al., 2014) and PAM (Steinthorsdottir et al., 2014; Thomsen et al., 2018).

Here, we describe the largest exome-array study to date across four commonly-used glycemic traits (fasting glucose [FG], fasting insulin [FI], glycated hemoglobin [HbA1c], and two-hour glucose [2hGlu]) in up to 144,060 non-diabetic individuals from multiple ancestries, to discover variants and loci influencing these traits within the physiological range. We sought to identify causal variants and putative effector transcripts in known and novel loci, and subsequently highlight pathways and tissues that are enriched for these glycemic trait associations. We further complemented our analyses with functional validation 
establish functional links between the associated rare coding variants in those loci and glucose regulation through different metabolic tissues. Together, our findings provide valuable insight into the biology underlying glycemic traits, and build on the knowledge required for validating candidate genes for therapeutic targeting in diabetes.

\section{Results}

\section{Identification of coding variant and gene-based glycemic trait associations}

We focused on coding variants on the exome chip as these could point more directly to their potential effector transcripts (i.e. likely causal gene[s]). Single-variant and gene-based association analyses with $\mathrm{FG}, \mathrm{FI}, \mathrm{HbA1c}$, and $2 \mathrm{hGlu}$ levels were performed on exome-array coding variants in up to 144,060 individuals without diabetes of European (85\%), African-American (6\%), South Asian (5\%), East Asian (2\%), and Hispanic (2\%) ancestry from up to 64 cohorts (Table S1, Methods).

We performed single-variant analyses in each individual cohort using a linear mixed model and combined results by fixed-effect meta-analyses within and across ancestries. As body mass index (BMI) is a major risk factor for T2D and is correlated with glycemic traits, all analyses were adjusted for BMI (Methods) to identify loci influencing glycemia independently from their effects on overall adiposity. We used distance-based clumping and considered signals to be novel, if they were located more than $500 \mathrm{~kb}$ from a variant with an established association with any of the glycemic traits or T2D at the time of the study (Methods).

Based on the above definition, we found 21 coding variants (in 18 genes) which were not previously associated with any other glycemic trait or T2D risk, that are now associated at exome-wide significance (defined as $P<2.2 \times 10^{-7}$ ) (Mahajan et al., 2018b; Sveinbjornsson et al., 2016) with their respective glycemic trait(s) (Table 1, Methods). Among these novel loci were a missense (p.E1365D) and splice region variant in OBSL1 associated with FI, another missense variant (p.L300P) in RAPGEF3 associated with $\mathrm{Fl}$, a missense variant ( $\mathrm{p} . \mathrm{S} 439 \mathrm{~N}$ ) in SPTB associated with $\mathrm{HbA1c}$, and missense variants $\mathrm{p} . \mathrm{R} 187 \mathrm{Q}$ in ANKH and p.R456Q in STEAP2 that are associated with FG (Table 1). OBSL1 encodes a cytoskeletal protein related to obscurin, mutations in which have been shown to lead to an autosomal recessive primordial growth disorder (OMIM: 612921). Loss of OBSL1 leads to downregulation of CUL7, a protein known to interact with IRS-1, downstream of the insulin receptor signaling pathway (Hanson et al., 
2009). RAPGEF3 encodes a cAMP-regulated guanine nucleotide exchange factor and is part of a cAMPresponsive signalling complex. The gene has been shown to be involved in cAMP-dependent adipogenesis (Jia et al., 2012), and investigation of associations in other related traits showed that the same RAPGEF3 variant is also associated with $\mathrm{BMI}$, waist-hip ratio and height (all $P<1 \times 10^{-4}$; Tables 1 and S2). This suggests that its role in adiposity and obesity is likely to link it to Fl regulation. Since the directions of effect of the variant are opposite for $\mathrm{FI}$ and BMI, the observed association could however be due to collider bias and should thus be interpreted with caution. SPTB encodes the protein spectrin beta, which is a major constituent of the cytoskeletal network underlying the erythrocyte plasma membrane. Mutations in this gene underlie a range of hematological disorders such as hemolytic anemias (OMIM: 617948, 616649). Given that red blood cell disorders can interfere with HbA1c levels (Wheeler et al., 2017a), this missense variant identifies SPTB as the likely effector transcript at this locus. ANKH encodes a transmembrane protein likely acting as a transporter. Recently, the FG-lowering allele reported here was shown to associate with decreased T2D risk in Europeans (OR=0.78 [0.69-0.87], $P_{\text {EUR }}=2.0 \times 10^{-7}$ ), and had a $>97 \%$ posterior probability of being causal, suggesting that this gene is the effector transcript at this locus (Mahajan et al., 2018b). In the final example, STEAP2 encodes a six transmembrane protein localized both intracellularly and on the plasma membrane, and is suggested to have roles in the regulation of iron transport (Sikkeland et al., 2016). A closely-related member of the STEAP family, STEAP4, has been reported to mediate cellular response to inflammatory stress through its role as a metalloreductase mediating iron and copper homeostasis (Scarl et al., 2017). Though little is known about STEAP2 function, in the recent T2D analysis, the FG-associated variant in STEAP2 was also found to be nominally associated with T2D risk $\left(P<1 \times 10^{-4}\right)$ (Mahajan et al., 2018b) (Tables 1 and S2). In addition to the novel loci, 53 other significant coding variant associations (in 40 genes) were detected that were within $500 \mathrm{~kb}$ of an established glycemic GWAS locus. These were of interest as they could point to a causal gene (Tables $\mathbf{1}$ and S3). sequence kernel association (SKAT) tests for gene-level analyses (Methods). We identified six genes with

117 significant evidence of association $\left(P<2.5 \times 10^{-6}\right)$, of which two $-G 6 P C$ (for FG and FI) and TF (for HbA1c) - represented novel associations (Tables 2 and S4). 
121 To establish whether the associated coding variants (both novel and those at established loci) were

122 likely to be causal, and/or likely to pinpoint an effector transcript, we first integrated these results with

123 published data with higher density GWAS coverage (Manning et al., 2012; Wheeler et al., 2017a). This is

124 important because coding variants can sometimes erroneously point to the wrong effector transcript, as

125 they can "piggy-back" on non-coding alleles that drive the association, and by virtue of having a

126 predicted effect on protein sequence they may falsely implicate the gene in which they reside as the

127 causal one. For example, the coding variant rs56200889 (p.Q802E) at ARAP1 is strongly associated with

128 FG $\left(\beta=-0.016, P=1.8 \times 10^{-14}\right.$, Table 1$)$, and when considered in isolation might have suggested ARAP1 as

129 the effector transcript. However, T2D fine-mapping efforts showed this association to be secondary to a

130 much stronger non-coding signal (Mahajan et al., 2018b), and recent data integrating human islet and

131 mouse knockout information has established neighbouring gene STARD10 as the most likely gene

132 mediating the GWAS signal at this locus (Carrat et al., 2017). Therefore, we conditioned the coding

133 variants identified here on existing non-coding GWAS index variants at established loci from two

134 previously published GWAS datasets (Manning et al., 2012; Wheeler et al., 2017a), and also performed

135 the reciprocal analysis (Table S3, Methods). At novel loci, we also assessed whether the coding variant

136 identified here was being driven by association of a sub-threshold (i.e. non genome-wide significant in

137 smaller sample size) non-coding variant based on published GWAS results with higher density coverage

138 (Manning et al., 2012; Wheeler et al., 2017a) (Methods). As reciprocal conditional analysis was not

139 always possible, or was not informative, we also used additional published data, including fine-mapping

140 results from comparable T2D efforts (Mahajan et al., 2018b), results for associations with blood cell

141 traits (Astle et al., 2016; Soranzo et al., 2010) (Table S5), as well as a body of literature establishing the

142 role of certain genes (mapping within our loci) in glucose metabolism, or red blood cell biology (for

$143 \mathrm{HbA1c}$ ) to inform effector transcript classification. We further considered significant gene-based

144 associations driven by multiple coding variants within a single gene as strong evidence for the

145 determination of effector transcripts (Methods).

147 Combining the above approaches, we curated the 74 coding variant associations (in 58 genes) displayed

148 in Table 1, and where possible identified and classified effector transcripts into "gold", "silver" and

149 "bronze" categories, depending on the strength of evidence (Table S6, Methods). Loci with strong

150 evidence from reciprocal conditional analysis or from published data that supported the relevance of

151 the identified effector transcript to the glycemic trait were labelled "gold" (e.g. GLP1R, SLC30A8, G6PD,

152 PPARG, ANK1); those where an effector transcript could not be defined by conditional analysis (either 
because it was inconclusive or due to lack of data) but where there was strong biological plausibility for a given gene at the locus were labelled "silver" (e.g. MADD, MLXIPL, FN3K/FN3KRP, HK1, VPS13C); those where we had some evidence but that was not as strong as "silver" were labelled "bronze" (e.g. DCAF12, OBSL1, STEAP2, RAPGEF3); the remaining were left with an undetermined effector transcript (Figure 1, Table S6). Effector transcript classification into the three categories was undertaken independently by four of the authors and the consensus was used as the final classification for effector transcripts. From 74 single variant and six gene-based signals, we identified 51 unique effector transcripts ( 24 gold, 11 silver, 16 bronze), with many of them shared across traits (Figure 1). One case in point pertains to VPS13C, which harboured a missense variant (p.R974K) associated with $2 \mathrm{hGlu}$ (labelled "bronze") at exome-wide significance $\left(\beta=-0.069, P=6.4 \times 10^{-10}\right.$; Table 1), and also exhibited a significant gene-based association with FG (labelled "silver"; $P_{\text {SKAT }}=3.7 \times 10^{-7}$; Table S4). VPS13C belongs to the previouslyestablished VPS13C/C2CD4A/C2CD4B glycemic trait and T2D risk locus, and recent follow-up studies have with varying levels of evidence suggested $C 2 C D 4 A$, encoding a calcium-dependent nuclear protein, as the causal gene for T2D through its potential role in the pancreatic islets (Kycia et al., 2018; Mehta et al., 2016; O'Hare et al., 2016). In our data, it is however not possible to rule out VPS13C as a potential effector transcript at this locus, warranting further functional studies for VPS13C, which encodes a protein reported to be necessary for proper mitochondrial function (Lesage et al., 2016).

\section{Pathway analyses identifies relevant gene sets regulating glycemia}

To identify pathways enriched for glycemic trait associations, and to subsequently determine the extent to which associations within the same trait implicate the same or similar pathways (as indicated by the functional connectivity of the network), we used GeneMANIA network analysis (Franz et al., 2018). GeneMANIA takes a query list of genes and finds functionally-similar genes based on large, publiclyavailable biological datasets. We analysed all loci harbouring non-synonymous variants that reached $P<1$ $\times 10^{-5}$ for any of the four glycemic traits in our study (totaling 121 associations). A high degree of connectivity was observed within the HbA1c network, with enrichment of processes related to blood cell biology such as porphyrin metabolism, erythrocyte homeostasis and iron transport (Figures $\mathbf{2}$ and S1, Table S7). In comparison, the network generated from FG-associated genes captured several processes known to contribute to glucose regulation and islet function, including insulin secretion, zinc transport and fatty acid metabolism (Figure 2, Table S7). The FG network further revealed linking nodes (that are not among the association signals) with known links to glucose homeostasis and diabetes, such as GCK (encoding the beta cell glucose sensor glucokinase), GCG (encoding the peptide hormone glucagon 

To identify additional candidate genes, we then performed heat map visualization with unsupervised

secreted by the alpha cells of the pancreas) and GIP (encoding the incretin hormone gastric inhibitory polypeptide). One gene within the FG cluster for lipid-related pathways is CERS2, which encodes ceramide synthase 2 , an enzyme known to be associated with the sphingolipid biosynthetic process (Figure 2, Table S7). Although CERS2 is only nominally associated with FG and is significantly associated with $\mathrm{HbA1c}$, it does not cluster together with any HbA1c-enriched pathway, suggesting that CERS2 is regulating FG and $\mathrm{HbA1c}$ indirectly through its role in lipid metabolism. Given that there were fewer genes associated with $\mathrm{Fl}$ and $2 \mathrm{hGlu}$, we were less powered to draw meaningful insights from the enriched pathways in those traits (Figure S1, Table S7).

We also performed gene set enrichment analysis (GSEA) using EC-DEPICT (Marouli et al., 2017; Turcot et al., 2018) (Methods). The primary innovation of EC-DEPICT is the use of 14,462 gene sets extended based on large-scale co-expression data (Fehrmann et al., 2015; Pers et al., 2015). These gene sets take the form of z-scores, where higher z-scores indicate a stronger prediction that a given gene is a member of a gene set. To reduce some of the redundancy in the gene sets (many of which are strongly correlated with one another), we clustered them into 1,396 "meta-gene sets" using affinity propagation clustering (Frey and Dueck, 2007). These meta-gene sets are used to simplify visualizations and aid interpretation of results. Here, we combined and analyzed all variants that reached $P<1 \times 10^{-5}$ for any of the four glycemic traits (Methods). We found 234 significant gene sets in 86 meta-gene sets with false discovery rate (FDR) of $<0.05$ (Table S8). As expected, we observed a strong enrichment of insulin- and glucoserelated gene sets, as well as exocytosis biology (in keeping with insulin vesicle release). In agreement with the GeneMANIA network analyses, we also noted a strong enrichment for blood-related pathways, which was primarily driven by $\mathrm{HbA1c}$-associated variants. This was likely because $\mathrm{HbA1c}$ levels are influenced not only by glycation but also by blood cell turnover rate (Cohen et al., 2008; Wheeler et al., 2017a). To disentangle blood cell turnover from effects due to glycation, we repeated the analysis excluding variants that were significantly associated with $\mathrm{HbA} 1 \mathrm{c}$ only and found 128 significant gene sets in 53 meta-gene sets (FDR <0.05) (Table S8). We also analyzed each of the four traits separately (Table S8, Methods). clustering of the membership predictions (z-scores) of trait-associated genes for each significant gene set (Figures 2, S2 and S3). This strategy has previously been effective for gene prioritization for downstream analyses (Marouli et al., 2017; Turcot et al., 2018), as it becomes visually apparent which 
genes are the strongest drivers of the significant gene sets and thus are natural targets for follow-up. This can be particularly helpful for prioritizing genes that are not well-characterized, as it leverages DEPICT's prediction of gene function. For the analysis of all traits except $\mathrm{HbA1c}$, one cluster showed particularly strong predicted membership for highly relevant gene sets, including "abnormal glucose homeostasis", "peptide hormone secretion", "Maturity Onset Diabetes of the Young", and multiple pathways involved in the regulation of glycogen, incretin, and carbohydrate metabolism (Figure $\mathbf{2 C}$ ). Strikingly, this cluster of six genes (PCSK1, GLP1R, GIPR, G6PC2, SLC30A8 and CTRB2) contained five of the genes that had independently been assigned to "gold" status during effector transcript identification (Table S6). Therefore, the sixth gene, CTRB2, represents a novel gene for prioritization, since it showed strong similarity to other genes for which there was already substantial biological evidence. CTRB2 encodes chymotrypsinogen B2, a digestive enzyme that is expressed in the exocrine pancreas, and subsequently secreted into the gut. The gene contains a borderline significant variant for $2 \mathrm{hGlu}$ (rs147238447; p.L6V; $\left.P=1.9 \times 10^{-6}\right)$. Another variant at this locus, rs7202877 (6.2kb downstream of CTRB2, $\mathrm{r}^{2}=0.0006, \mathrm{D}^{\prime}=1$ with $\mathrm{rs} 147238447$ in European populations), has previously been shown to be an eQTL for CTRB1 and CTRB2, with the minor $\mathrm{G}$ allele $(\mathrm{MAF}=11 \%)$ associated with increased expression ( $\mathrm{t}$ Hart et al., 2013). In the same study, the rs7202877-G allele was associated with increased glucagon-like peptide 1 (GLP-1)-stimulated insulin secretion $\left(P=8.8 \times 10^{-7}, \mathrm{~N}=196\right)$. In our data, rs7202877-G was nominally associated with lower $2 \mathrm{hGlu}\left(P=6.3 \times 10^{-3}\right)$ and lower FG $\left(P=2.8 \times 10^{-3}\right)$ levels. Multiple distinct signals in this region (previously referred to as the BCAR1 locus) have also been associated with T2D risk, including rs7202877 (where the G allele is protective), rs72802342 $\left(r^{2}=0.65\right.$ with rs 7202877 in European populations) and rs3115960, although the coding variant rs147238447 described here is not (Mahajan et al., 2018a; Mahajan et al., 2018b; Morris et al., 2012; Zhao et al., 2017). This can potentially be explained by limited power to identify a significant association given the low MAF ( $0.5 \%)$ of the coding variant. In contrast to its effect on T2D, the rs7202877-G allele has been associated with increased risk of type 1 diabetes (OR=1.28, $P=3.1 \times 10^{-15}, \mathrm{~N}=21,293$ ) (Barrett et al., 2009). Other variants at this locus are associated with risk of chronic pancreatitis ( $r s 8055167, r^{2}=0.0021$ with $r s 147238447$ and $r^{2}=0.12$ with rs7202877 in European populations, in LD with an inversion that changes the expression ratio of CTRB1 and CTRB2 isoforms) (Rosendahl et al., 2017) and pancreatic cancer (rs7190458, $\mathrm{r}^{2}=0.0002$ with rs147238447 and $r^{2}=0.31$ with rs7202877 in European populations) (Wolpin et al., 2014). The prioritization of CTRB2 is intriguing as it supports an emerging hypothesis that the exocrine pancreas contributes to complex mechanisms influencing $2 \mathrm{hGlu}$ levels and diabetes risk (Esteghamat et al., 2019; Hart et al., 2018; Woodmansey et al., 2017). Given the earlier associations with GLP-1 stimulated insulin 
secretion, we investigated whether this effect could be mediated by incretin levels. However, we found no associations at rs147238447 for GLP-1 levels in the largest available dataset (fasting GLP-1, N=4170: $\mathrm{MAF}=0.00457, P=0.495 ; 2 \mathrm{~h} G \mathrm{LP}-1, \mathrm{~N}=3839$ : $\mathrm{MAF}=0.00464, P=0.076$ ) (Almgren et al., 2017), though this might again be explained by limited power. Although additional validation of the rare coding variant rs147238447 (p.L6V) as a potential causal variant is required given the absence of clear associations with T2D risk and other glycemic traits, the results discussed above suggest a role of CTRB2 in glycemic regulation.

We also noted a small but distinct cluster in the FG-only analysis indicating the role of the cilium/axoneme, pointing to novel biology relating to sensing and signaling in response to the extracellular environment (Figure 2D). Two genes were the main drivers of this association: WDR78 and $A G B L 2$. These represent potentially interesting candidates for follow-up, although we note that the $A G B L 2$ signal may be driven through effects of the nearby MADD gene, which harbors a FG-associated coding variant in our study and is labelled "silver" in our effector transcript classification (Tables $\mathbf{1}$ and S6). Overall, our network and pathway analyses highlighted several trait-associated genes that do not reach exome-wide significance in conventional single variant or gene-based tests, but show evidence of contribution to glycemic regulation.

\section{Tissue enrichment analysis reveals shared roles of key tissues in the regulation of glycemic traits}

In addition to identifying key metabolic pathways involved in glucose regulation, we sought to establish the relative importance of particular tissues in the regulation of the different glycemic phenotypes. This time, we assessed the tissues that are most highly enriched for the expression of the 51 effector transcripts we have curated at the associated loci identified in this study, to highlight specific tissues that contribute critically to the regulation of each glycemic trait. Using publicly-available tissue expression data from GTEx (Battle et al., 2017) and human islets (van de Bunt et al., 2015), we noted clear differences in tissue enrichment patterns as well as tissues shared between traits (Figure 3). Comparisons between analyses of FG- and Fl-associated effector transcripts underscored the relative roles of the liver in both traits $(P<0.05)$, whereas pancreatic islets were enriched in associations for FG $\left(P=9.99 \times 10^{-5}\right)$ but not FI $(P=0.75)$. In contrast, adipose $(P=0.01)$ and kidney tissues $(P=0.01)$ were enriched in $\mathrm{Fl}$ but not FG $(P>0.05)$. These results not only highlight the established role of pancreatic islets in influencing FG levels, but also the under-appreciated role of insulin clearance in the kidney and 
likely the liver, in addition to insulin action in liver and adipose tissue, in influencing FI levels (Goodarzi et al., 2011). Consistent with the EC-DEPICT GSEA, there was also support for the role of the exocrine pancreas (which typically represents $>95 \%$ of whole pancreas tissue) in addition to the endocrine pancreas (islets) in FG $\left(P=9.99 \times 10^{-5}\right)$ and $2 \mathrm{hGlu}\left(P=2.99 \times 10^{-4}\right)$ associations. We also observed enrichment for genes expressed in stomach for $2 \mathrm{hGlu}\left(P=1.99 \times 10^{-4}\right)$ but not for FG $(P=0.16)$. HbA1c analysis revealed enrichment in "metabolic" tissues reflecting insulin secretion (islets, $P=1.59 \times 10^{-2}$ and pancreas, $P=0.01$ ), insulin action (muscle, $P=1.50 \times 10^{-2}$ ), insulin clearance (liver, $P=0.03$ ), as well as strong enrichment for whole blood $\left(P=3.99 \times 10^{-3}\right)$. These indicate key factors relating to hemoglobin glycation and blood cell function in influencing overall HbA1c levels (Figure 3).

Our results from the pathway and tissue enrichment analyses demonstrate the role of specific tissues with known functions in blood glucose regulation in particular glycemic traits. These observations add further support to emerging reports of an underappreciated role for the exocrine pancreas in FG and $2 \mathrm{hGlu}$ regulation, the stomach-incretin axis in $2 \mathrm{hGlu}$, and the importance of insulin clearance through the kidney and liver in FI.

\section{Novel glycemic trait associations in liver-enriched G6PC are driven by functional coding}

\section{variants}

To delve deeper into tissue-specific gene effects, we focused on two homologues, G6PC and G6PC2, with constrasting tissue expression profiles where we identified gene-based association signals for FG/FI and FG/HbA1c, respectively (Tables 2 and S4). Both genes encode gluconeogenic enzymes that catalyze the same biochemical pathway but are known to have distinct tissue expression profiles. G6PC2 is largely expressed in pancreatic islets whereas G6PC is highly expressed in the liver, kidney, and small intestine (Foster et al., 1997; Mithieux, 1997). Our gene-based analyses highlighted G6PC through novel associations with FG and FI, driven primarily by rare missense variants p.A204S (rs201961848) and p.R83C (rs1801175), and protein-truncating variant (PTV) p.Q347X (rs80356487), none of which achieved exome-wide significance at single-variant level (Table S4). Homozygous inactivating alleles in G6PC, which include both p.R83C and p.Q347X, are known to give rise to glycogen storage disease type la (GSD1a), a rare autosomal recessive metabolic disorder (Chou and Mansfield, 2008; Lei et al., 1995), but this is the first time that rare coding variants in G6PC have been shown to influence FG and Fl levels in normoglycemic individuals. 
311 Given the well-known role of G6PC in hepatic glucose homeostasis, we were interested in elucidating

312 the molecular impact of rare heterozygous G6PC coding variants highlighted in our exome-array

313 analysis, in particular novel variant p.A204S, one of the statistical drivers of the gene-based G6PC signal

314 (Table S4). In transient protein overexpression assays, p.R83C and p.A204S resulted in significantly

315 reduced protein levels compared to wild type (WT) G6PC in both Huh7 (human hepatoma) and HEK293

316 (human embryonic kidney) cell lines (Figure 4A-D). The PTV p.Q347X, which in our in vitro system

317 generated a smaller molecular weight protein, exhibited markedly lower protein expression levels in

318 Huh7 cells but not HEK293 cells. However, in both cell types, the cellular localization pattern of p.Q347X

319 appears to be largely diffuse and did not co-localize with the Golgi apparatus, which is important for

320 post-translational modification of G6PC protein (Figures 4E and S4A). Further functional characterization

321 of glucose-6-phosphatase (G6Pase) activity revealed that both p.R83C and p.Q347X variants lead to

322 proteins lacking any detectable phosphatase activity (Figure S4B-C), consistent with previous

323 observations of several GSD1a-causing coding variants (Shieh et al., 2002). As we observed that the

324 p.R83C variant resulted in complete loss of glycosylation, we determined if glycosylation is essential for

325 G6Pase activity by treating cells with tunicamycin to inhibit N-linked glycosylation. The ability of

326 unglycosylated G6PC to catalyze glucose-6-phosphate (G6P) was found to be downregulated by up to

327 14\%, although this difference was not statistically significant (Figure 4F). We therefore concluded that

328 whilst glycosylation contributes to overall functional activity, it may not be a requisite for G6P

329 hydrolysis. Finally, we were unable to accurately assess p.A204S-G6PC phosphatase activity as the level

330 of expression in the microsomes was reduced by $41 \%$ relative to WT, supporting the hypothesis that

331 p.A204S-G6PC exhibits partial loss-of-function (LOF) most likely due to loss of protein expression.

Together, our functional studies support p.A204S, p.R83C, and p.Q347X as functional LOF variants due

334 to loss of G6Pase protein expression and/or activity. This results in a reduced potential to hydrolyze G6P

335 to glucose in gluconeogenic tissues (such as in the liver and kidney), thus directly reducing FG levels and

336 consequently lowering circulating Fl levels in the plasma. Our data suggest that rare inactivating

337 mutations in G6PC (such as p.R83C and p.Q347X) that cause the autosomal recessive disorder GSD1a

338 can also modulate fasting glycemic traits within a normoglycemic range in asymptomatic heterozygous

339 variant carriers.

340

341

G6PC2 alleles influence protein function by multiple mechanisms 
342

343

344

345

346

347

348

349

350

351

352

353

354

355

356

357

358

359

360

361

362

363

364

365

366

367

368

369

370

371

372

373

G6PC2, a gene homolog of G6PC, is an established effector transcript at a GWAS locus which contains multiple coding variants known to influence FG and HbA1c but not Fl levels (Bouatia-Naji et al., 2008; Chen et al., 2008; Mahajan et al., 2015; Soranzo et al., 2010; Wessel et al., 2015). In this current study, gene-based association signals for both FG and HbA1c were observed at the G6PC2 locus, primarily driven by multiple coding variants (p.H177Y, p.Y207S, p.R283X, and p.S324P) (Table S4). We aimed to extend the investigation of coding variation in this gene, which is likely to harbor a series of functional alleles, by characterizing the four G6PC2 coding variants above and six others, across the allelic frequency spectrum (all with single-variant $P<0.05$ for FG or HbA1c in our analyses) (Table S4; Figure S5A). Protein overexpression studies in the rat insulinoma cell line INS-1 832/13 and HEK293 cells revealed that seven of the G6PC2 variants characterized (including PTV p.R283X) resulted in significantly reduced protein levels (Figures 5A and S5B-C). In INS-1 832/13 cells, this effect was largely due to partial or total loss of the glycosylated form of the protein. In HEK293 cells, the reduction in total protein levels could be rescued when the proteasomal pathway (but not the lysosomal pathway) was inhibited, consistent with an earlier study involving a smaller subset of variants (Mahajan et al., 2015), confirming proteasome-mediated protein turnover.

As three variants (p.I171T, p.I171V, and p.F256L) appeared to be stably expressed and processed like WT G6PC2 protein, we hypothesized that these alleles could be influencing glycemic levels through effects on protein activity. As there is a high level of conservation between the catalytic domains in G6PC and G6PC2, we adapted the G6Pase assay used earlier, to indirectly analyse the effect of the G6PC2 variants on G6Pase enzymatic activity. We assumed that the G6PC2 alleles of interest, which mapped to the conserved regions, will give rise to the same consequence in the G6PC backbone due to the strong homology and preserved topology of both proteins. The adaptation was necessary as we were unable to detect G6PC2 activity using the same experimental conditions. First, we generated variants that mapped to equivalent sites within the G6PC protein (G6PC-p.L173T, p.L173V, and p.F258L correspond to G6PC2p.I171T, p.I171V, and p.F256L, respectively), and then performed the enzymatic studies. Two alleles, p.L173T, p.L173V, affected the same codon and were each genetically associated with FG levels but with opposite directions of effect (Table S4). We found that G6PC-p.L173T exhibited $\sim 20 \%$ decreased activity compared to WT based on assessment of $\mathrm{V}_{\max }$ (maximal rate of reaction), a measure of enzymatic activity (Figure 5B). In contrast, G6PC-p.L173V had enhanced activity through both increased $\mathrm{V}_{\max }$ and lowered $\mathrm{K}_{\mathrm{m}}$ (Michaelis constant, whereby a lower $\mathrm{K}_{\mathrm{m}}$ indicates higher substrate affinity) (Figure 5B). Importantly, our in vitro observations mirrored the genetic effects on $\mathrm{FG}\left(\beta_{1171 \mathrm{~T}}=-0.084 \mathrm{mmol} / \mathrm{l}\right.$; 
$\left.374 \quad \beta_{1171 \mathrm{~V}}=+0.131 \mathrm{mmol} / \mathrm{l}\right)$ and HbA1c levels $\left(\beta_{1171 \mathrm{~T}}=-0.007 \% ; \beta_{1171 \mathrm{~V}}=+0.093 \%\right)$ (Table S4). The G6PC-p.F258L

375 variant also displayed impaired phosphatase activity due to reduced $\mathrm{V}_{\max }$ and a tendency towards higher

$376 \mathrm{~K}_{\mathrm{m}}$ relative to WT (Figure 5C), consistent with the observed glucose-lowering effects of G6PC2-p.F256L.

377 To ensure that the observed effects of the rare variants on FG were not influenced by the common

378 G6PC2 variant rs560887, as was the case for a common variant V219L shown in an earlier study

379 (Mahajan et al., 2015) which we confirm here, conditional analyses were performed conditioning on

380 rs560887 (Table S9). Conditional results for p.I171T, p.I171V and p.F256L confirmed that the directions

381 of effect for the variants remain unchanged, making it unlikely that the regulatory variant rs560887 is

382 regulating these effects (Table S9). These results provided the first example of an activating allele in

383 G6PC2 (p.I171V) and highlighted the unique protein changes at a single codon that can give rise to a

384 corresponding loss or gain of functional activity. These data therefore show that variations in G6PC2

385 may influence FG levels through their impact on protein expression or activity.

To further characterize these variants, we set out to determine the effect of the G6PC2 LOF variants on ER integrity, given that G6PC2 is an ER-resident protein and that beta cells, which are highly-specialized secretory cells, are highly sensitive to ER stress. Specifically, we evaluated the expression of G6PC2 variant proteins on the canonical ER stress response (ERSE) and unfolded protein response (UPRE) pathways. The three G6PC2 variants which displayed relatively severe effects on protein stability (p.H177Y, p.Y207S, p.S324P) in our study were found to activate ERSE and UPRE reporter activities by 3-fold, in contrast to the variants p.I171T and p.F256L which exert their effects primarily on enzymatic function (Figure 5D). The common p.V219L variant, which reduces protein expression by approximately $50 \%$, displayed an intermediary effect (Figure 5D). These results suggest that G6PC2 variant proteins, especially those that result in severe LOF due to protein instability, may also influence beta cell ER 397 homeostasis.

In previous studies, the G6PC2-p.R283X variant has shown inconsistencies in terms of their associations with FG levels (Mahajan et al., 2015; Wessel et al., 2015). With a larger dataset we have now confirmed 401 that this variant influences both FG and HbA1c levels (Tables 1 and S3). As the nonsense p.283X allele is 402 located in the last exon of the gene and may evade NMD, we queried RNA sequencing data from human 403 islets and observed an allelic balance in heterozygous carriers, indicating that variant transcripts are 404 indeed likely to escape NMD and be translated (Figure S6A). Based on our pipeline of in vitro assays, we 
confirmed G6PC2-p.R283X loss-of-function due to reduced protein expression, failure to localize to the Golgi network, and a high likelihood of complete loss of phosphatase activity (Figures 5A and S5D).

In contrast to the mechanisms in play for the coding variants in G6PC2, the non-coding GWAS index variant at the G6PC2 locus (rs560887) is suggested to influence expression of G6PC2 splice variants based on previous minigene analyses in HeLa cells (Baerenwald et al., 2013; Bouatia-Naji et al., 2010). To establish whether this variant indeed influences G6PC2 regulation in human islets, we determined its effect on G6PC2 isoform expression. We found that in human islets, the presence of the rs560887-G allele is associated with increased expression of the full-length G6PC2 isoform as compared with the shorter isoform lacking exon 4 (Figure S6B). This observation supports the hypothesis that rs560887 may alter splicing and is consistent with the association between rs560887-G and elevated FG and HbA1c levels due to increased G6PC2 function. As the phenotypic consequence of rare coding variants can be influenced by regulatory variants on the same haplotype, we therefore performed conditional analyses to explore the relationship between rs560887 and the rare coding variants. We showed that the direction of effects of all the rare alleles in our study remained the same after conditioning on rs560887, though it is notable that the variants p.Y207S and p.R283X showed some reduction in strength of association after conditioning (Table S9).

\section{Functional assessment of G6PC2 variants improves gene-based association analysis}

We next evaluated the utility of our functional data to enhance gene-based association analyses. We showed that the gene-based signals were strengthened when the tests were informed by in vitro functional validation of the variants (as determined in this study) as opposed to the predictive in silico annotations based on the NSbroad and NSstrict masks (Table S9, Methods). In fact, in line with expectation, flipping the alleles in the gain-of-function variant p.I171V (which we now know acts in the opposite direction compared to other rare variants in the test), to align all alleles with the same direction of effect, augmented the strength of association for both FG (from $P=4.34 \times 10^{-71}$ to $P=6.47 \times$ $\left.10^{-78}\right)$ and $\mathrm{HbA1C}\left(P=6.37 \times 10^{-30}\right.$ to $\left.P=6.37 \times 10^{-33}\right)$ in the gene burden test (Table S9). Improved methods of filtering variants will enhance the performance of gene-based tests and increase the likelihood of identifying true association signals, especially for those that are of borderline significance or that initially fall below the significance threshold.

\section{G6PC2 regulates basal insulin secretion in human beta cells}


437 Although G6PC2 is known to be specifically enriched in pancreatic islet beta cells, its role in the

438 regulation of human beta cell function has not been shown. Using gene knockdown studies in the

439 human EndoC- $\beta \mathrm{H} 1$ beta cell line, we found that G6PC2-deficient cells exhibited significantly (but

440 modestly) increased insulin secretion at low glucose $(1 \mathrm{mM})$ and a trend towards increased insulin

441 secretion at sub-maximal glucose $(6 \mathrm{mM})$ levels (Figures $\mathbf{5 F}$ and S5E). When expressed as a fraction of

442 insulin content (Figure 5F), insulin secretion was significantly increased across multiple glucose

443 conditions, although this was primarily driven by reduced total insulin content in G6PC2-deficient cells

444 by 15\%. Overall, G6PC2 knockdown increases glucose responsiveness at sub-threshold levels of glucose

445 but not at maximal glucose concentration in EndoC- $\beta \mathrm{H} 1$ cells, suggesting enhancement of basal glucose

446 sensitivity by promoting glycolytic flux at sub-stimulatory glucose concentrations, and warranting more

447 in-depth characterization experiments. 


\section{Discussion}

449 We have identified novel coding variant associations with $\mathrm{FG}, \mathrm{Fl}, 2 \mathrm{hGlu}$ and $\mathrm{HbA1c}$, across the allele

450 frequency spectrum, and assigned these variants to their effector transcripts using available genetic and

451 biological evidence. We further pinpointed novel loci and effector transcripts that have now been

452 associated with T2D and other related metabolic traits since the time of our analysis. Our results

453 revealed that 15 out of 58 glycemic trait-associated loci have evidence of association with T2D risk

454 (Table S2) (Hara et al., 2014; Mahajan et al., 2018b; Williams et al., 2014). For instance, FG-associated

455 loci ANKH and STEAP2, and HbA1c-associated DCAF12 all associate with T2D risk (Table S2), providing

456 opportunities to investigate the mechanisms through which associated variants influence both glycemic

457 regulation within the physiological range as well as T2D pathophysiology. The Fl-associated RAPGEF3

458 locus is also associated with various obesity-related measures including BMI and WHR, potentially

459 supporting our tissue enrichment analyses linking FI with adiposity.

460 We used this work to explore the pathways and metabolic tissues through which the associated genes

461 influence variation in glycemic traits and highlighted those with key roles in glucose regulation and traits

462 that act through multiple metabolic tissues, including islets, liver, fat, and in addition, exocrine pancreas,

463 gut and kidney. Our GSEA enabled us to identify additional genes (e.g. CTRB2) within these tissues and

464 pathways which were below the threshold for statistical significance in our initial discovery effort and

465 that merit follow-up. We report an emerging role for the gut and exocrine pancreas for $2 \mathrm{hGlu}$ levels and

466 potentially T2D risk through multiple analyses, consistent with current understanding that both incretins

467 and digestive enzymes are important in controlling postprandial glucose levels (Esteghamat et al., 2019;

468 Hart et al., 2018; Woodmansey et al., 2017). We also show that different traits are influenced by

469 pathways operating in distinct tissues: FG is almost exclusively influenced by pathways in the endocrine

470 and exocrine pancreas and liver, whilst Fl is mediated by the insulin-sensitive tissues such as liver,

471 kidney, and adipose tissue, indicating the importance of both insulin action and insulin clearance

472 mechanisms. Genes expressed in muscle, also an insulin-sensitive tissue, were enriched in HbA1c-

473 associated effector loci but not $\mathrm{FI}$, though this could be due to differences in power between the two

474 analyses. We see evidence of multiple metabolic tissues being important for $\mathrm{HbA} 1 \mathrm{c}$ regulation, and note

475 that the HbA1c-associated set of effector transcripts appear enriched for those that influence blood cell

476 biology.

477 We have also shown for the first time that genetic variation in G6PC, a gene implicated in GSD1a,

478 influences glycemic traits within the normal physiological range in heterozygote carriers. In vitro follow- 
up of the variants driving the gene-based association - p.A204S, p.R83C, and p.Q347X - confirmed that these were indeed causal LOF variants at this locus that contribute to modulation of FG and Fl levels. We then reported novel rare coding variant associations for $\mathrm{FG}$ and $\mathrm{HbA} 1 \mathrm{c}$ within a member of the same gene family, G6PC2, and expanded the allelic spectrum of reported variants to include variants affecting the same codon with both loss and gain of function alleles. Our comprehensive analysis of this locus demonstrates multiple molecular mechanisms by which variants influence protein function, including evidence from human islets that the common regulatory variant rs560887 influences G6PC2 isoform expression, and that a rare PTV ( $p$. R283X) evades NMD and results in a catalytically-null enzyme. Given the possiblility that the effects of any coding variants in exon 4 which are carried in cis with the rs560887-A allele could potentially be "diluted" due to the splicing effect, we checked whether the observed rare variant effects could be driven by rs560887 in LD by repeating the single-variant association tests with conditional analyses (Table S9). In our analysis, the directions of effect of the rare coding alleles do not appear to be influenced by the non-coding regulatory allele. We then used our in vitro data to refine existing methods for gene-based association analysis to demonstrate the value of functional data in improving their sensitivity. New developments in high-throughput functional annotation that can overcome the time-consuming nature of functional experiments will greatly facilitate such efforts (Liu et al., 2017; Tewhey et al., 2016; Ulirsch et al., 2016). Finally, to understand how loss of G6PC2 influences FG levels, we silenced it in a human beta cell model and demonstrated increased insulin secretion at low glucose levels, in line with the genetic observations.

It has long been suspected that particular metabolic tissues are key to governing specific processes of glucose metabolism. Using human genetics, our study has explored this within an unbiased approach and has illustrated the impact of altered glycolysis in multiple metabolic tissues on various glycemic phenotypes. Uniquely, our parallel studies of G6PC and G6PC2 highlighted two homologous proteins that act through different tissues to influence glycemic traits. As G6PC is involved in hepatic glucose production it influences both FG and Fl levels. Previous studies have also established a potential role for G6PC in influencing lipid and urate levels (Dewey et al., 2016; Sever et al., 2012). In contrast, due to its restricted expression in the islet beta cell, variants in G6PC2 only influence FG and HbA1c due to a beta cell-driven effect. There are also notable differences in the molecular mechanisms underlying protein dysfunction: for G6PC variants the effect is primarily on enzymatic activity, whilst G6PC2 variants largely cause protein instability. 
511 A limitation of the present study is that we were not able to fine-map association signals, being

512 restricted to variants captured on the exome array, leaving many associated loci with unknown effector

513 transcripts. Additional large-scale studies, with higher density GWAS arrays and imputation to dense

514 reference panels, will be required for fine-mapping and further effector transcript identification.

515

516 In conclusion, we have combined human genetic discovery with pathway analysis and functional studies

517 to uncover tissue-specific effects in common pathways that influence glycemic traits. Our findings will

518 inform efforts to target these pathways therapeutically to modulate metabolic function. 


\section{Figure Legends}

Figure 1. Effector transcript classification into "gold", "silver" and "bronze" categories based on strength of genetic and biological evidence. A total of 51 effector transcripts from 74 single variant and six gene-based signals were identified, with many of them shared across traits. The classification was undertaken independently by four of the authors and the consensus was used as the final classification for effector transcripts (see Methods). *Asterisk indicates "silver" for FG, "bronze" for 2 hGlu. different methods for variant associations with $P<1 \times 10^{-5}$. (A-B) The networks represent composite networks for (A) HbA1c and (B) FG, from the GeneMANIA analysis using genes with variant associations at $P<1 \times 10^{-5}$ for each trait as input. Nodes outlined in red correspond to genes from the input list. Other nodes correspond to related genes based on 50 default databases. Based on the network, GO terms and Reactome pathways that were significantly enriched are depicted. To summarize these results, the most significant term of all calculated terms within the same group is represented. Barplots with the Bonferroni-adjusted -log10(p-values) of the most significant terms within each group are are shown. Each group was assigned a specific color; if a gene is present in more than one term, it is displayed in more than one color.

537 (C-D) Heatmaps showing EC-DEPICT results from analysis of (C) all traits except HbA1c and (D) FG. The

538 columns represent the input genes for the analysis. In (C), these are genes with variant associations of $539 P<1 \times 10^{-5}$ for $\mathrm{FG}, \mathrm{Fl}$, and/or $2 \mathrm{hGlu}$, and in (D) these are genes with variant associations of $P<1 \times 10^{-5}$ for

540 FG. Rows in the heatmap represent significant meta-gene sets (FDR $<0.05)$. The color of each square 541 indicates DEPICT's z-score for membership of that gene in that gene set, where dark red means "very

542 likely a member" and dark blue means "very unlikely a member." The gene set annotations indicate 543 whether that meta-gene set was significant at FDR $<0.05$ or not significant (n.s.) for each of the other EC-

544 DEPICT analyses. For heatmap intensity and EC-DEPICT $P$-values, the meta-gene set values are taken

545 from the most significantly enriched member gene set. The gene variant annotations are as follows: (1)

546 the European minor allele frequency (MAF) of the input variant, where rare is $M A F<1 \%$, low-frequency

547 is MAF 1-5\%, and common is MAF $>5 \%$, (2) whether the gene has an Online Mendelian Inheritance in

548 Man (OMIM) annotation as causal for a diabetes/glycemic-relevant syndrome or blood disorder, (3) the

549 effector transcript classification for that variant: gold, silver, bronze, or NA (note that only array-wide 550 significant variants were classified, so suggestively-significant variants are by default classified as "NA"), 
551 (4-7) whether each variant was significant $\left(P<2 \times 10^{-7}\right)$, suggestively significant $\left(P<1 \times 10^{-5}\right)$, or not

552 significant in Europeans for each of the four traits, and (8) whether each variant was included in the analysis or excluded by filters (see Methods). AWS: array-wide significant. Related to Figures S1 to S3.

Figure 3. Tissue enrichment analysis reveals the key tissues involved in the regulation of glycemic

traits. The figures display expression enrichment of genes from all of the golden, silver, and bronze gene set lists for (A) HbA1c, (B) FG, (C) FI and (D) 2hGlu in GTEx tissue samples plus islet data. Enrichment $P$ values were assessed empirically for each tissue using a permutation procedure $(10,000$ iterations), and the red vertical line shows the significance threshold (empirical $P<0.05$ ).

Figure 4. Functional characterisation of G6PC variant proteins. Related to Figure S4. HEK293 cells $(n=5)$ by western blot densitometric analysis of FLAG-tagged G6PC constructs relative to

564 tubulin control, with representative blots shown.

565 (C) Protein expression levels of PTV Q347X were determined in Huh7 cells ( $n=3$ ) and (D) HEK293 cells

$566(n=4)$ by western blot densitometric analysis of V5-tagged G6PC constructs relative to tubulin control,

567 with representative blots shown. Bars in red indicate variants that are statistical drivers of the gene-

568 based signal.

569 (E) Cellular localisation of V5-tagged G6PC-Q347X was assessed in Huh7 cells and overlaid with markers

570 for the ER (calreticulin) and the trans-Golgi network (TGN46). White arrows point to positions of the 571 Golgi apparatus. Scale bar indicates $10 \mu \mathrm{m}$.

572 (F) Glucose-6-phosphatase activity of unglycosylated WT G6PC protein obtained from tunicamycin-

573 treated (Tuni) HEK293 microsomes ( $n=2)$, with representative western blot of microsomal protein

574 shown. All data presented as mean \pm SEM. ${ }^{*} p=0.01-0.05 ; * * p=0.001-0.01 ;{ }^{* *} p<0.001$. cells. Related to Figure S5.

578 (A) Expression levels of the glycosylated forms (upper bands only) of G6PC2 variant proteins were 579 determined in INS-1 832/13 cells by western blot densitometric analysis of Myc-tagged G6PC2 580 constructs relative to tubulin control $(n=5)$. Representative blots are shown for untreated cells together 581 with cells treated with proteasomal inhibitor MG-132 or lysosomal inhibitor chloroquine. 
582 (B) Glucose-6-phosphatase activity of L173T and L173V variants in G6PC (proxy for I171T and I171V in

583 G6PC2 respectively) in HEK293 against increasing glucose-6-phosphate concentrations ( $n=4$ ), with mean

$584 \quad V m a x \pm S E M$ and $\mathrm{Km} \pm$ SEM values shown for WT and each variant.

585 (C) Glucose-6-phosphatase activity of F258L variant in G6PC (proxy for F256L in G6PC2) in HEK293

586 against increasing glucose-6-phosphate concentrations ( $n=3)$, with mean Vmax $\pm S E M$ and Km \pm SEM

587 values shown. Vmax and Km results were computed based on the Michaelis-Menten kinetic model.

588 (D) Effect of G6PC2 WT and variant protein expression on luciferase activity driven by ER stress response

589 elements in HEK293 cells. Relative luciferase units corrected for background activity were normalised to

590 WT for each reporter, from $n=6$ across two independent experiments (except for F256L, $n=3$ in one

591 experiment) using two-way ANOVA with Fisher's LSD test comparing each variant to WT.

592 (E) Cellular localisation of R283X in EndoC- $\beta \mathrm{H} 1$ overlaid with markers for the ER (calreticulin) and the

593 trans-Golgi network (TGN46). White arrows point to positions of the Golgi apparatus. Scale bar indicates $59410 \mu \mathrm{m}$.

595 (F) Insulin secretion normalised to total content at basal and high glucose conditions (with and without

596 drug treatments) following 96-120h G6PC2 knockdown in EndoC- $\beta H 1$. Unpaired two-tailed Students' t

597 tests were used to compare G6PC2 knockdown to control for each condition, from n=16 across 4

598 independent experiments. Tol: tolbutamide; Diaz: diazoxide. All data presented as mean \pm SEM. *

$599 \mathrm{p}=0.01-0.05 ; * * \mathrm{p}=0.001-0.01 ; * * * \mathrm{p}<0.001$.

600

601 
602 Table Legends

603

604 Table 1. Single-point coding variant associations meeting the significant threshold for coding variants

605 of $\boldsymbol{P}<\mathbf{2 . 2} \times \mathbf{1 0 ^ { - 7 }}$. This table includes all novel coding variants meeting this threshold, irrespective of

606 whether they fall in completely new loci or in previously-established loci, provided that the association

607 at the established locus was not shown to be due to a non-coding variant (Table S3) or another coding

608 variant at the same locus. Novel loci are highlighted in bold. HbA1c: glycated haemoglobin; FG: fasting

609 glucose; Fl: fasting insulin; $2 \mathrm{hGlu}$ : 2h glucose; Alleles E/O: effect allele/other allele; Freq. Effect Allele:

610 frequency of effect allele; Effect (SE): effect size (standard error); P: p-value; N: number of samples in

611 the analysis; Novel/previous glycemic trait association: Novel corresponds to a new association result;

612 Locus name of previous association - name used for previously-reported locus. ${ }^{1}$ Significant in the

613 European-only analysis in our study. ${ }^{2}$ Genome-wide significant association with T2D since date of

614 analysis (Mahajan et al., 2018b). ${ }^{3}$ Association with T2D at $P<1 \times 10^{-4}$ since date of analysis (Mahajan et al.,

615 2018b). ${ }^{4}$ T2D locus identified in Japanese (Hara et al.,2014) and Mexican (Williams et al., 2014)

616 populations only. The date of our exomes analysis is May 2015. Related to Table S3.

Table 2. Gene-based results from broad (NSbroad mask) and strict (NSstrict mask) analyses. Genes in bold are newly discovered from this effort. $\mathrm{N}$ var: total number of variants in that gene-based analysis; $P_{\text {burden: }} p$-value from burden test which assumes all variants have the same direction of effect; $P_{\text {SKAT }}: p$ -

621 value from SKAT test which allows for different directions of effect between variants. The lowest $p$-value

622 is highlighted in bold. Related to Table S4. 
623

624

625

626

627

628

629

630

631

632

633

634

635

636

637

638

639

640

641

642

643

644

645

646

647

648

649

650

651

652

\section{Methods}

\section{LEAD CONTACT AND MATERIALS AVAILABILITY}

Further information and requests for resources and reagents should be directed to and will be fulfilled by the Lead Contacts, Inês Barroso (ines.barroso@mrc-epid.cam.ac.uk) and Anna L Gloyn (anna.gloyn@drl.ox.ac.uk).

\section{EXPERIMENTAL MODEL AND SUBJECT DETAILS}

\section{Studies in humans}

MAGIC (Meta-Analysis of Glucose and Insulin-related traits Consortium) was established to focus on the genetic analysis of glycemic traits in individuals without diabetes. In this MAGIC effort, non-diabetic individuals of European (85\%), African-American (6\%), South Asian (5\%), East Asian (2\%) and Hispanic (2\%) ancestry from up to 64 cohorts participated. Sample sizes were up to 144,060 for HbA1c, 129,665 for FG, 104,140 for Fl and 57,878 for 2hGlu. Participating cohorts and their characteristics are detailed in Table S1.

\section{Studies in cellular models}

HEK293 cells were cultured in Dulbecco's Modified Eagle's Medium (DMEM) (D6429, Sigma Aldrich), $10 \%$ (v/v) foetal bovine serum (FBS) (10500-064, Life Technologies), $100 \mathrm{U} / \mathrm{ml}$ penicillin and $100 \mathrm{\mu g} / \mathrm{ml}$ streptomycin (15140122, Life Technologies). Huh7 cells were cultured in DMEM (31885, Life

45 Technologies), 10\% (v/v) FBS, $100 \mathrm{U} / \mathrm{ml}$ penicillin and $100 \mathrm{\mu g} / \mathrm{ml}$ streptomycin. INS-1 832/13 cells were cultured in Roswell Park Memorial Institute-1640 (RPMI-1640) media (R0883, Sigma Aldrich), 10\% (v/v) FBS, $100 \mathrm{U} / \mathrm{ml}$ penicillin and $100 \mu \mathrm{g} / \mathrm{ml}$ streptomycin, $2 \mathrm{mM}$ L-glutamine (25030081, Life Technologies), 1 mM sodium pyruvate (S8636, Sigma Aldrich), 10 mM HEPES (H3537, Sigma Aldrich), 50 HM 2mercaptoethanol (Life Technologies). EndoC- $\beta \mathrm{H} 1$ cells were cultured in DMEM (31885, Life Technologies), Bovine Serum Albumin (BSA) fraction V (10775835001, Roche), $100 \mathrm{U} / \mathrm{ml}$ penicillin and $100 \mu \mathrm{g} / \mathrm{ml}$ streptomycin, $2 \mathrm{mM}$ L-glutamine, $50 \mu \mathrm{M}$ 2-mercaptoethanol, $10 \mathrm{mM}$ nicotinamide (Sigma Aldrich), $5.5 \mu \mathrm{g} / \mathrm{ml}$ transferrin (Sigma Aldrich) and $6.6 \mathrm{ng} / \mathrm{ml}$, sodium selenite (Sigma Aldrich). All cell 
653

654

655

656

657

658

659

660

661

662

663

664

665

666

667

668

669

670

671

672

673

674

675

676

677

678

679

680

681

682

683

lines were tested negative for mycoplasma contamination using the MycoAlert Assay kit (Lonza). Cells were maintained at $37^{\circ} \mathrm{C}$ and $5 \% \mathrm{CO}_{2}$.

\section{METHOD DETAILS}

\section{Studies in humans}

Phenotypes

Studied outcomes were FG (mmol/L), Ln-transformed FI (pmol/L), 2hGlu (mmol/L) and HbA1c (\% of hemoglobin). Glycemic measurements are described in detail for each contributing cohort in Table S1. Individuals with diagnosed or treated diabetes, or those with diabetes on the basis of $F G(\geq 7 \mathrm{mmol} / L)$, $2 \mathrm{hGlu}((\geq 11.1 \mathrm{mmol} / \mathrm{L})$ and/or HbA1c $(\geq 6.5 \%)$ were excluded from analyses.

\section{Genotyping and QC}

The Illumina HumanExome BeadChip is a genotyping array containing variants that have been observed in sequencing data of $\sim 12,000$ individuals. Non-synonymous variants seen at least three times across at least two datasets were included on the exome chip. More lenient criteria were used for splice and nonsense variants. Besides the core content of protein-altering variants, the exome chip contains additional variants including common variants identified in GWAS, ancestry informative markers, mitochondrial variants, randomly selected synonymous variants, HLA tag variants and Y chromosome variants. In this study we analysed association with glycemic traits of 247,470 autosomal and X chromosome variants present on the exome chip. Genotype calling and quality control were performed following protocols developed by the UK Exome Chip or CHARGE consortium (Grove et al., 2013). The exact genotyping array, calling algorithm and QC procedure used by each cohort are depicted in Table S1.

\section{Annotation and functional prediction of variants}

Annotation of the exome chip variants was performed using the Ensembl Variant Effect Predictor v78. In silico functional prediction from SIFT, Polyphen HumDiv, Polyphen HumVar, LRT and MutationTaster was added using dbNSFP v2.9 (Liu et al., 2013; Yourshaw et al., 2015).

\section{Statistical analyses}

Single variant analyses 
Individual cohorts ran linear mixed models using the raremetalworker (v 4.13.2) or rvtests (v20140723) software (Table S1). For each glycemic outcome, analyses were performed using an additive model for the raw and the inverse normal transformed trait. In the manuscript and in all tables and figures effect estimates and standard errors are for the raw trait, while the $p$-values are from the inverse normal transformed trait analyses. Analyses were adjusted for age, sex, BMI, study-specific number of PCs and other study-specific covariates (Table S1). Raremetal (v4.13.7 or higher) was used to combine results by fixed-effect meta-analyses. Variants with $P<10^{-4}$ for deviation from Hardy-Weinberg equilibrium or with call rate $<0.99$ in individual cohorts were excluded from meta-analyses. In single variant analyses, the threshold for significance was $P<2.2 \times 10^{-7}$ for coding variants (stop-gained, stop lost, frameshift, splice donor, splice acceptor, initiator codon, missense, in-frame indel and splice region variants). These $P$ value thresholds were based on a Bonferroni correction weighted by the enrichment for complex trait associations among the different functional annotation categories (Mahajan et al., 2018b; Sveinbjornsson et al., 2016). Significant association signals located more than $500 \mathrm{~kb}$ from any variant already known to be associated with the trait at the time of analysis (May 2015) were considered novel for the trait.

\section{Gene-based analyses}

In addition, raremetal was used to perform gene-based burden and sequence kernel association (SKAT) tests. For both burden and SKAT tests, two in silico masks for inclusion of variants in the test were used: NSstrict and NSbroad. The NSstrict mask includes PTVs (splice donor, splice acceptor, stop gained, frameshift, stop lost or initiator codon variant) OR variants that are missense and predicted to be damaging by five prediction algorithms (SIFT, Polyphen HumDiv, Polyphen HumVar, LRT, MutationTaster). The NSbroad mask additionally includes missense variants predicted to be damaging by at least one of the five prediction algorithms AND that have a MAF $<1 \%$ in each ancestry group. These MAFs were derived from our single variant HbA1c meta-analyses results ( $N$ up to 144,060 ). For G6PC2, we also used masks filtering on functional variants that have been determined in vitro to influence protein expression or function. The $P$-value threshold for significance in gene-based analyses was $2.5 \times 10^{-6}$ (Bonferroni correction for 20,000 genes).

\section{Conditional analyses}

Approximate conditional analyses were performed using Raremetal v 4.13.8. At known glycemic trait loci, if previously known GWAS index variants (or good proxies) were present on the exome chip, 
significant lead coding variants were conditioned on these known index variants and vice versa to identify distinct coding variant signals. At novel loci, to identify additional distinct associated variants, analyses were performed conditioning on the most significant variant at the locus. These analyses were repeated by including the next most significant and distinct associated variant until no exome- or genome-wide significantly-associated variants were left at the locus. For gene-based signals, to identify the variants driving the signal, analyses were performed conditioning on the variant with the most significant $p$-value that was included in the mask. These analyses were repeated including the next most significant variant until association at the gene was attenuated $(P>0.05)$. If there were both gene-level and known or novel single variant associations at the same locus (within $500 \mathrm{~kb}$ ), we additionally conditioned on the associated single variant to assess whether the gene-based association was distinct from the single variant association.

\section{Putative effector transcript identification}

To identify putative effector transcripts, at known glycemic trait loci we considered the transcript a putative effector transcript if there was a distinct coding variant signal (still meeting the threshold for significance of $P<2.2 \times 10^{-7}$ after conditioning on the non-coding GWAS index variant, for details on these conditional analyses methods refer to the conditional analyses methods section above). Coding variant associations at novel loci were followed up on in published GWAS results with higher density coverage (Manning et al., 2012; Wheeler et al., 2017a). If the coding variant was present in the GWAS results, approximate conditional analyses were performed using GCTA (Yang et al., 2012). If the GWAS index variant signal was abolished by conditioning on the coding variant, we considered this as evidence supporting the transcript as a putative effector transcript. If the both the GWAS index variant and the coding variant signals were attenuated, the results were considered uninformative and we considered the transcript in light of other data. We additionally utilized published data to classify effector transcripts, including (1) fine-mapping results from comparable T2D efforts (Mahajan et al., 2018b) and (2) a body of literature establishing a role in glucose metabolism or red blood cell biology (for HbA1c) for certain genes that mapped within our loci. Significant gene-based associations driven by multiple coding variants within a single gene, in particular where an impact on protein expression or function could be demonstrated, were considered strong evidence for the determination of effector transcripts. Combining these approaches, we attempted to identify effector transcripts at each locus, and we classified their likelihood of being correct depending on the strength of the evidence. Those effector transcripts where there was strong evidence from reciprocal conditional analysis or support from 
published data for the relevant glycemic trait or phenotype were labelled "gold"; those where the effector transcript could not be defined by conditional analysis (either because it was inconclusive or due to lack of data) but where there was strong biological plausibility for a given gene at the locus were labelled "silver"; those where we had some tentative evidence but that was not strong enough to warrant a "silver" classification were labelled "bronze", and the remainder were left with an unknown effector transcript. Effector transcript classification into "gold", "silver" and "bronze" was undertaken independently by four of the authors and the highly concordant consensus score was given (Table S6).

\section{GeneMANIA network analysis}

For network analyses, we used GeneMANIA (v3.5.1), a network approach that searches many large, publicly-available biological datasets to find related genes. These include protein-protein, protein-DNA and genetic interactions, pathways, reactions, gene and protein expression data, protein domains and phenotypic screening profiles. Briefly, GeneMANIA uses a label propagation algorithm for predicting gene function given the composite functional association network (calculated from the databases selected). The weights needed for the label propagation method to work are selected at the beginning of the process. In our case, and according to the defaults, we weighted the network using linear regression, to make genes in the input list interact as much as possible with each other. We analyzed all non-synonymous variants for each locus with a cut-off of association $P<1 \times 10^{-5}$ with any trait (input genes). We performed four network analyses: (1) HbA1c-associated variants only, (2) Fl-associated variants only, (3) FG-associated variants only, and (4) 2 hGlu-associated variants only.

We selected the 50 default databases to create the composite network, and we allowed the method to find at most 50 genes that are related to our query input list. The resultant networks were investigated to find enriched Gene Ontology (GO) terms and Reactome Pathways. Gene Set Enrichment (GSE) of networks and sub-networks were assessed with ClueGO (Bindea et al., 2009) using GO terms and Reactome gene sets (Croft et al., 2014). The enrichment results were grouped using a Cohen's Kappa score of 0.4 , and terms were considered significant with a Bonferroni-adjusted $p$-value $<0.05$, provided that there was an overlap of at least three network genes in the relevant GO gene set when calculating GO enrichment. For the pathway selection (Reactome), we set a threshold that the network genes should represent at least $4 \%$ of the pathway. These values were applied given the recommended defaults when running ClueGO (Bindea et al., 2009). Cohen's Kappa statistic was used to measure the gene-set similarity of GO terms and Reactome pathways and allowed us to group enriched terms into 
779

780

781

782

783

784

785

786

787

788

789

790

791

792

793

794

795

796

797

798

799

800

801

802

803

804

805

806

807

808

functional groups to improve visualization of enriched pathways. We used all genes with GO annotations and at least one interaction in our network database as the background set.

\section{Gene set enrichment analysis (GSEA)}

For GSEA, we used EC-DEPICT, an extension of the GWAS GSEA method DEPICT (Pers et al., 2015). ECDEPICT has been described elsewhere (Marouli et al., 2017; Turcot et al., 2018). Briefly, the key feature of EC-DEPICT is the use of "reconstituted" gene sets, which are gene sets collected from many different databases (e.g. canonical pathways, protein-protein interaction networks, and mouse phenotypes) that have been extended based on large-scale microarray co-expression data (Fehrmann et al., 2015; Pers et al., 2015).

Six groups of variants were analyzed: (1) HbA1c-associated variants only, (2) Fl-associated variants only, (3) FG-associated variants only, (4) 2hGlu-associated variants only, (5) all trait-associated variants, and (6) all trait-associated variants except for HbA1c (see Methods). For each trait, we clumped the European summary statistics (+/- $500 \mathrm{~kb}$ on either side). Then, the most significant nonsynonymous variant for each locus was included in the analysis, with a cut-off of $P<10^{-5}$. Annotations from the CHARGE consortium were used to assign variants to genes (see URL). After GSEA, highly correlated gene sets were grouped by affinity propagation clustering of all 14,462 gene sets (Frey and Dueck, 2007) into "meta-gene sets" using SciKitLearn.clustering.AffinityPropagation version 0.17 (Abraham et al., 2014). For all visualizations, the gene set within a meta-gene set with the best enrichment $P$-value was used; heat maps were created with the ComplexHeatmap package in $\mathrm{R}$ (Gu et al., 2016).

URL: CHARGE Consortium ExomeChip annotation file (v6):

http://www.chargeconsortium.com/main/exomechip/

Method and choice of data for permutations: We performed the EC-DEPICT analysis as described elsewhere (Marouli et al., 2017; Turcot et al., 2018). All analyses are based on a group of 14,462 "reconstituted" gene sets, which contains a z-score for probability of gene set membership for each gene (for details, see (Fehrmann et al., 2015; Pers et al., 2015)).

Briefly, the basic EC-DEPICT method is as follows. We first obtain a list of significant input variants (the most significant nonsynonymous variant per locus) and then map variants to genes based on annotations from the CHARGE consortium (see URL). For each gene set, we obtain the gene set 
809

810

811

812

813

814

815

816

817

818

819

820

821

822

823

824

825

826

827

828

829

830

831

832

833

834

835

836

837

membership z-scores for all trait-associated input genes and sum them to generate a test statistic. We then take 2,000 permuted ExomeChip association studies (described in more detail below) and calculate the average permuted test statistic for that gene set, as well as the permuted standard deviation. For each permutation, the number of top genes we take as "input genes" is matched to the actual observed number of input genes. We then calculate (observed test statistic - average permuted test statistic)/(permuted standard deviation) to generate a z-score, which is converted to a $p$-value via the normal distribution. False discovery rates were calculated by comparing the observed $p$-values to a permuted $P$-value distribution generated with an additional set of 50 permuted association studies.

The permuted ExomeChip association studies are conducted by (1) generating 2,200 sets of normally distributed phenotypes and (2) using these randomly generated phenotypes to conduct 2,200 association studies with real ExomeChip data. Using these permutations to adjust the observed test statistics corrects for any inherent structure in the data (e.g. that pathways made up of longer genes may be more likely to come up as significant by chance).

For these analyses, we first generated permutations based on ExomeChip data we had used previously for this purpose: 11,899 samples drawn from three cohorts (Malmö Diet and Cancer [MDC], All New Diabetics in Scania [ANDIS], and Scania Diabetes Registry [SDR]). For simplicity, we refer to these cohorts as the "Swedish permutations."

As part of our GSEA pipeline, we remove input trait-associated variants that are not present in the permuted data to ensure that all variants are appropriately modeled. When using the Swedish permutations, this generally results in removing a substantial fraction of the variants, especially of the very rarest variants (due to the smaller sample size of the Swedish data relative to the data being analyzed). We have previously observed that this filtering can actually improve the GSEA signal, possibly due to more heterogeneous biology or a higher false-positive rate in these very rare variants (Turcot et al., 2018). However, in this case, we observed that in performing this filtering, we excluded variants in several known monogenic disease genes, such as HNF1A and SLC2A2. Therefore, we wished to repeat the analysis with a set of permutations which would allow us to retain these variants. We thus repeated the analysis with a second set of permutations consisting of 152,249 samples from the UK Biobank (referred to as the "UKBB permutations"). The larger sample size in the UKBB permutations means more variants are present and can therefore be included in the analysis. 
completeness, we report the results from the use of both sets of permutations. We note that the results are strongly concordant. The larger number of significant gene sets reported based on the UK Biobank permutations is generally a combination of 1 ) overall improved power (i.e. more variants are included)

842 and 2) the inclusion of variants in key driver genes absent in the Swedish permutations, encompassing 843 both the monogenic genes mentioned above (e.g. SLC2A2) and additional genes with clearly relevant

844 biology (e.g. CTRB2, SLC3OA8). The results from both sets of permutations are summarized below. For all analyses, "significance" refers to a false discovery rate of $<0.05$.

All-trait analysis: After filtering, 78 input genes were included for the analysis with the UKBB permutations and 60 for the analysis with the Swedish permutations. (Note that the difference in the number of input genes is due to the presence of a larger number of input variants in the UKBB permutations - see above). We found 234 significant gene sets in 86 meta-gene sets based on the UKBB permutations (Figure S2) and 133 gene sets in 51 meta-gene sets based on the Swedish permutations

(Figure S3). The correlation between the UKBB and Swedish analyses was $r=0.902, P<10^{-300}$.

All-traits-except-HbA1c analysis: After filtering, 45 input genes were included for the analysis with the UKBB permutations and 33 for the analysis with the Swedish permutations. We found 128 significant gene sets in 53 meta-gene sets based on the UKBB permutations (Figure S2) and 45 significant gene sets in 18 meta-gene sets based on the Swedish permutations (Figure S3). The correlation between the UKBB and Swedish analyses was $r=0.882, P<10^{-300}$. permutations and 33 for the analysis with the Swedish permutations. We found 191 significant gene sets in 73 meta-gene sets based on the UKBB permutations (Figure S2) and 120 gene sets in 41 meta-gene sets based on the Swedish permutations. (Figure S3). The correlation between the UKBB and Swedish analyses was $r=0.936, P<10^{-300}$.

FG-only analysis: After filtering, 26 input genes were included for the analysis with the UKBB permutations and 22 for the analysis with the Swedish permutations. We found 106 significant gene sets in 39 meta-gene sets based on the UKBB permutations (Figure S2) and 48 significant gene sets in 15

867 meta-gene sets based on the Swedish permutations (Figure S3). The correlation between the UKBB and 868 Swedish analyses was $r=0.939, P<10^{-300}$. 
2hGlu-only analysis: After filtering, 12 input genes were included for the analysis with the UKBB permutations and 7 for the analysis based on the Swedish permutations. We found 56 significant gene sets in 17 meta-gene sets based on the UKBB permutations (Figure S2), with no significant gene sets

872 based on the Swedish permutations. The correlation between the UKBB and Swedish analyses was $r=$ $0.787, P<10^{-300}$.

874 Fl-only analysis: After filtering, 11 input genes were included for the analysis with the UKBB

875 permutations and 8 for the analysis with the Swedish permutations. There were no significant gene sets

876 from either analysis. The correlation between the UKBB and Swedish analyses was $r=0.860, P<10^{-300}$.

877 Visualization: As in previous work (Marouli et al., 2017; Turcot et al., 2018), we have included all trait878 associated variants in the heat maps, even if they were excluded from the analysis (e.g. because they were absent in the permutations or did not have a nonsynonymous annotation in the CHARGE annotation file). This is because we assume that if the genes harboring those variants have strong predicted membership in significantly trait-associated gene sets, they are still good candidates for

882 prioritization. In fact, this may be even stronger evidence in favor of these genes because they did not contribute to the enrichment analysis and therefore their prioritization is independently derived (and provides even more support to the implicated biology).

\section{Tissue enrichment analysis}

We analysed identified genes (all 51 effector transcripts) for tissue enrichment using publicly available expression data from the GTEx project, version 7 and publicly-available islet expression data (van de Bunt et al., 2015). We use transcripts per million (TPM) values for gene level analyses. We have excluded genes from non-coding proteins and only used those with unique HGCN IDs ( $n=20,160)$. We ranked all genes by median TPM across all samples, and generated 10,000 permutations of each gene set list (golden, silver, and bronze) by selecting a random gene for each entry in the gene set within \pm 150 ranks of the transcript for that gene. For each sample in GTEx tissues, the TPM values were converted into ranks for that gene, and sums of ranks within each tissue were computed for each gene. We calculated

895 enrichment $p$-values for each tissue by taking the total number of instances when the gene list of interest had a lower sum of ranks than the permuted sum of ranks (divided by the total number of permutations). To check that our results were not driven by sample size differences in each of the 45 analyzed GTEx tissues and islet tissue, we applied a 'downsampling' strategy. We performed 3 different 
downsampling analyses with 100, 150 and 175 samples chosen at random from each of the selected GTEx tissues and compared them to the results obtained with the whole dataset. During each downsampling round, we only used those tissues with at least the target number of samples (100, 150 or 175) because the random selection was performed under a no-replacement condition. Our results were robust to sample size differences and the trends observed were not driven by differences in

904 sample sizes across tissues.

905

906

RNA-sequencing of human islets

907 RNA from human islet samples ( $\mathrm{n=150)}$ was sequenced on Illumina HiSeq2000 as previously described

908 (van de Bunt et al., 2015). Allele-specific expression was assessed using MAMBA (Pirinen et al., 2015).

909 For the isoform effects, all protein-coding and lincRNA transcripts from GRCh37 (Ensembl release 75)

910 were quantified using Salmon v0.8.1 (Patro et al., 2017). Isoform ratios were calculated by dividing each

911 transcript's expression by the total expression of that gene. For the QTL analysis, all isoforms with

912 expression in $<50 \%$ or all samples, with no variance between samples, only 0 or 1 fractions across

913 samples, or those originating from non-autosomal chromosomes were removed. Ratios of the remaining 914 transcripts were rank-transformed to normality. Subsequently, 30 PEER factors to account for potential 915 sources of non-genetic noise were derived from the normalized isoform ratios, and, together with three 916 genotype principal components and a sex covariate, were used in the QTL analysis using FastQTL (Ongen 917 et al., 2016). Finally, the resulting beta-approximated $p$-values were adjusted for multiple testing across 918 all tested transcripts using the Benjamini-Hochberg procedure.

\section{Studies in cellular models}

921

922 Site directed mutagenesis. Human G6PC (NM_000151.3) and G6PC2 cDNA (NM_021176.2) within a

923 pCMV6-Entry vector (with a C-terminal Myc-FLAG-tag) was purchased from OriGene (RC215623 and

924 RC211146 respectively). For the study of PTVs, an N-terminal V5 tag sequence (5'-

925 GGTAAGCCTATCCCTAACCCTCTCCTCGGTCTCGATTCTACG-3') was cloned into the OriGene vectors. Single

926 nucleotide substitutions were generated in the G6PC or G6PC2 coding sequence using Quikchange II

927 Site-Directed Mutagenesis (Agilent). All mutations were verified by Sanger sequencing and in each case, only the desired nucleotide changes were introduced. 
929 Western blot analyses. Western blots were performed on total protein lysates collected from human

930 HEK293 and Huh7 cells and rat INS-1 832/13 cells transfected with each wild type or mutant

931

932

933

934

935

936

937

938

939

940

941

942

943

944

945

946

947

948

949

950

951

952

953

954

955

956

957

958

959 G6PC/G6PC2 construct using Lipofectamine 2000 (Invitrogen) according to manufacturer's instructions. All cell lines were tested negative for mycoplasma contamination. For the inhibition of cell proteolysis,

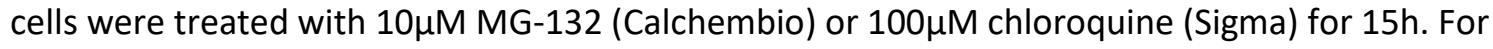
inhibition of $\mathrm{N}$-linked glycosylation, cells were treated with $1 \mu \mathrm{g} / \mathrm{ml}$ tunicamycin (Sigma) for $15 \mathrm{~h}$. Cells were collected 36-48h after transfection and homogenized in lysis buffer. Cell lysates were separated by 4-12\% SDS-PAGE (Bio-Rad/Invitrogen). The antibodies used for determining recombinant G6PC/G6PC2 expression were: anti-FLAG M2 (Sigma, F1804), anti-V5 (Invitrogen, 46-0705) or anti-myc 4A6 (Millipore, 05-724). A $\beta$-tubulin antibody (Santa Cruz, sc-9104) was used as a loading control. Secondary antibodies specific to mouse or rabbit IgG were purchased from Thermo Fisher Scientific. Protein bands were detected using the western enhanced chemiluminescence substrate (BioRad).

Immunofluorescence microscopy. Human HEK293, Huh7 and EndoC- $\beta$ H1 cells were transfected using FuGene 6 transfection reagent (Promega) according to manufacturer's instructions, in 4-well chamber slides (BD Biosciences). After 48h, cells were fixed with 4\% paraformaldehyde in PBS, permeabilized with $0.05 \%$ Triton X-100 in PBS and blocked with 10\% BSA in PBS-Tween 20. Double immunostaining of cells was carried out using anti-FLAG M2 (Sigma, F1804) or anti-V5 (Invitrogen, 46-0705), together with anticalreticulin (Thermo, PA3-900) or anti-TGN46 (Sigma, T7576) primary antibodies. The secondary antibodies used were anti-mouse Alexa Fluor 488 and anti-rabbit Alexa Fluor 568, both from Life Technologies. DRAQ5 fluorescent probe (Thermo Fisher Scientific) was applied at $20 \mu \mathrm{M}$ as a far-red nuclear stain. Finally, slides were mounted with ProLong Gold antifade reagent (Life Technologies) and visualized on a BioRad Radiance 2100 confocal microscope with a 60X 1.0 N.A. objective. Images were acquired with different laser settings that were optimized for each sample and therefore fluorescent intensities are not comparable across samples.

Glucose-6-phosphatase activity of microsomal samples. For the collection of microsomes, HEK293 cells were transfected with $12.5 \mu \mathrm{g}$ of wild type or mutant G6PC construct in $10 \mathrm{~cm}$ dishes using Lipofectamine 2000 (Invitrogen). For the study of G6PC2 variant activity, site directed mutagenesis was carried out within the conserved sequence regions on the G6PC background. Cells were cultured in Dulbecco's modified Eagle's medium (DMEM) supplemented with $10 \%$ fetal bovine serum, $100 \mathrm{U} / \mathrm{ml}$ penicillin, and $100 \mu \mathrm{g} / \mathrm{ml}$ streptomycin. At least two dishes of cells per condition were harvested $48 \mathrm{~h}$ after transfection and scraped into $0.25 \mathrm{M}$ sucrose- $5 \mathrm{mM}$ HEPES buffer $(\mathrm{SH})$ followed by several rinses in 
SH. Cells were mechanically homogenized using a Potter-Elvehjem glass tissue grinder and Teflon pestle, followed by 12 passes through a 27-gauge syringe needle. The homogenate was subjected to centrifugation at $10,000 \mathrm{~g}$ for $10 \mathrm{~min}$ and the supernatant (post-nuclear fraction) was further centrifuged at 100,000 g for $1 \mathrm{~h}$ in a TLA 100.4 rotor in an Optima TLX ultracentrifuge (Beckman Coulter). A pellet containing the microsomal fraction was obtained and resuspended in $\mathrm{SH}$. An aliquot of each microsomal sample was lysed in lysis buffer for protein quantification using the Bradford reagent (BioRad) and analysed by western blot (BioRad) to determine the relative levels of recombinant G6PC WT and variant expression. For glucose-6-phosphatase assays, matched amounts of G6PC WT or variant

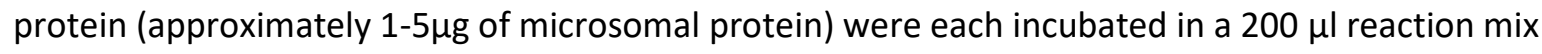
containing $100 \mathrm{mM} \mathrm{MES} \mathrm{pH} \mathrm{6.5,} \mathrm{and} 0$ to $20 \mathrm{mM} \mathrm{G6P}$ at $37^{\circ} \mathrm{C}$ for $8 \mathrm{~min}$. The reaction was terminated by addition of $20 \%$ trichloroacetic acid and centrifuged at 4,000 rpm for $10 \mathrm{~min}$ in a microcentrifuge. The supernatant was mixed in equal parts with a Taussky-Shorr colour reagent ( $1 \%$ ammonium molybdate, $5 \%$ iron(II) sulphate heptahydrate in $0.5 \mathrm{M} \mathrm{H}_{2} \mathrm{SO}_{4}$ ) for $7.5 \mathrm{~min}$ before measuring absorbance at $660 \mathrm{~nm}$ on a spectrophotometer (Molecular Devices Ltd). The amount of phosphates detected was calculated using a $\mathrm{KH}_{2} \mathrm{PO}_{4}$ standard curve. Results were expressed as mean normalised activity ( $\mathrm{nmol} / \mathrm{mg} / \mathrm{min}$ ) relative to the activity of wild type at $20 \mathrm{mM}$ G6P for every experiment. Finally, Michaelis-Menten enzyme kinetic analysis and paired t tests of determined kinetic constants were carried out on GraphPad Prism 6.0.

ER stress response reporter assays. HEK293 cells were co-transfected with G6PC2 WT or variant constructs and pGL3-Promoter constructs containing ER stress response elements (ERSE-I and ERSE-II) or UPR elements (UPRE-P and UPRE-W) using the FuGene 6 transfection reagent (Promega). A Renilla luciferase gene-containing pRL-CMV was also co-transfected as an internal transfection control. Cells were lysed in passive lysis buffer (Promega) and assayed using the Dual Luciferase Assay System (Promega).

Insulin secretion analysis in EndoC- $\beta \mathrm{H} 1$ cells. Gene knockdown was carried out on EndoC- $\beta \mathrm{H} 1$ cells using ON-TARGETplus siRNA (Dharmacon, GE Healthcare) and Lipofectamine RNAiMAX (Life Technologies) at a final concentration of $25 \mathrm{nmol} / \mathrm{L}$ siRNA. For static incubation experiments, cells were placed in 2.8 mM glucose DMEM (11966, Gibco by Life Technologies) overnight. Cells were starved in 0 $\mathrm{mM}$ glucose medium for $1 \mathrm{~h}$ the following day, then stimulated in DMEM containing $1 \mathrm{mM}$ glucose, 6 mM glucose, 20 mM glucose, 20 mM glucose with $100 \mu \mathrm{M}$ tolbutamide (Sigma Aldrich) or $20 \mathrm{mM}$ glucose with $100 \mu \mathrm{M}$ diazoxide (Sigma Aldrich) at $37^{\circ} \mathrm{C}$ for $1 \mathrm{~h}$. Each condition was carried out in triplicate 
991

992

993

994

995

996

997

998

999

1000

1001

1002

1003

1004

1005

1006

1007

1008

1009

1010

1011

1012

1013

1014

1015

1016

1017

or quadruplicate wells within each experiment. Viable cell count was measured using the CyQUANT Direct Cell Proliferation Assay kit (C35012, Thermo Scientific). All cell count values were expressed as fluorescent units normalised to mean cell count at $1 \mathrm{mM}$ glucose. Cells were extracted for analysis of insulin content with cold $\mathrm{HCl}$-ethanol (Sigma Aldrich). Insulin levels were measured using the human insulin AlphaLISA detection kit (AL204C, Perkin Elmer).

\section{QUANTIFICATION AND STATISTICAL ANALYSIS}

Western blot bands for protein expression studies were quantified by densitometry analysis using Image and densitometric data between G6PC/G6PC2 WT and each variant from 3-5 independent experiments were compared using two-tailed paired Students' $t$ tests. For enzymatic assays, mean differences in activity between G6Pase WT protein and each variant protein for the substrate G6P were compared using two-tailed unpaired Students' $t$ tests of the determined kinetic constants $V_{\max }$ and $K_{m}$. For the analysis of ER stress luciferase activity data, a two-way analysis of variance (ANOVA) was applied to compare mean fold difference in reporter activity between G6PC WT and variant. For gene expression analyses, G6PC2 KO and control cells were analysed using two-tailed unpaired Students' $\mathrm{t}$ tests. For the analysis of insulin secretion data, mean differences between G6PC2 KO cells and control cells for each condition or time point were compared using two-tailed unpaired Students' t tests. Plotting of graphs and statistical analyses were carried out on GraphPad Prism 6.0 or 7.0. A P value $<0.05$ was considered significant.

\section{Acknowledgements}

HepG2 cells were the kind gift of the Tomlinson group (OCDEM), INS-1 832/13 cells were the kind gift of Jochen Lang (Université de Bordeaux), and EndoC- $\beta \mathrm{H} 1$ cells were gifted by Raphael Scharfmann (INSERM).

\section{Study/Individual $\quad$ Acknowledgment}

1958 British Birth Analysis was supported by BHF programme grant (Deloukas) RG/14/5/30893 
cohort

AGES

Andrei I. Tarasov

Andrew P Morris

Anna L. Gloyn

ARIC

ASCOT
This study has been funded by NIH contracts N01-AG-1-2100 and 271201200022C, the NIA Intramural Research Program, Hjartavernd (the Icelandic Heart Association), and the Althingi (the Icelandic Parliament). The study is approved by the Icelandic National Bioethics Committee, VSN: 00-063. The researchers are indebted to the participants for their willingness to participate in the study. Andrei I. Tarasov acknowledges the support from Oxford Biomedical Research Centre (OxBRC fellowship)

Andrew P Morris is a Wellcome Trust Senior Fellow in Basic Biomedical Science. ALG is a Wellcome Trust Senior Fellow in Basic Biomedical Science. This work was funded by the Wellcome Trust (095101/Z/10/Z and 200837/Z/16/Z).

The Atherosclerosis Risk in Communities (ARIC) study is carried out as a collaborative study supported by the National Heart, Lung, and Blood Institute (NHLBI) contracts (HHSN268201100005C, HHSN268201100006C, HHSN268201100007C, HHSN268201100008C, HHSN268201100009C, HHSN268201100010C, HHSN268201100011C, and HHSN268201100012C). The authors thank the staff and participants of the ARIC study for their important contributions. Funding support for "Building on GWAS for NHLBI-diseases: the U.S. CHARGE consortium" was provided by the NIH through the American Recovery and Reinvestment Act of 2009 (ARRA) (5RC2HL102419). ML was supported by a National Heart, Lung, and Blood Institute T32-HL0072024 Cardiovascular Epidemiology Training Grant.

This work was supported by Pfizer, New York, NY, USA, for the ASCOT study and the collection of the ASCOT DNA repository; by Servier Research Group, Paris, France; and by Leo Laboratories, Copenhagen, Denmark. We thank all ASCOT trial participants, physicians, nurses, and practices in the participating countries for their important contribution to the study. In particular we thank Clare Muckian and David Toomey for their help in DNA extraction, storage, and handling. This work forms part of the research programme of the NIHR Cardiovascular Biomedical Research Unit at Barts 
Athero-Express

Biobank Study

BioMe

Caroline Hayward

CHS
Sander W. Van der Laan is funded through grants from the Interuniversity Cardiology Institute of the Netherlands (ICIN, 09.001), CVON (GENIUS), CVgenesat-Target (EU FP7/2007-2013 under grant agreement $n^{\circ}$ HEALTH-F2-2013-601456). Claudia Tersteeg, Krista den Ouden, Mirjam B. Smeets, Sander M. van de Weg, Noortje A.M. van den Dungen, and Loes B. Collé are graciously acknowledged for their work on the DNA extraction. Evelyn Velema, Sara van Laar, Arjan H. Schoneveld, and Petra H. Homoed-van der Kraak are graciously acknowledged for their past and continuing work on the Athero-Express Biobank Study. We would also like to thank all the (former) employees involved in the Athero-Express Biobank Study of the Departments of Surgery of the St. Antonius Hospital Nieuwegein and University Medical Center Utrecht for their continuing work. Jessica van Setten is graciously acknowledged for her help in the quality assurance and quality control of the genotype data. Lastly, we would like to thank all participants of the Athero-Express Biobank Study; without you these kinds of studies would not be possible.

The Mount Sinai BioMe Biobank is supported by The Andrea and Charles Bronfman Philanthropies.

Caroline Hayward is supported by an MRC University Unit Programme Grant "QTL in Health and Disease" MC_PC_U127592696.

This CHS research was supported by NHLBI contracts HHSN268201200036C, HHSN268200800007C, HHSN268201800001C, N01HC55222, N01HC85079, N01HC85080, N01HC85081, N01HC85082, N01HC85083, N01HC85086; and NHLBI grants U01HL080295, R01HL068986, R01HL087652, R01HL105756, R01HL103612, R01HL120393, and U01HL130114 with additional contribution from the National Institute of Neurological Disorders and Stroke (NINDS). Additional support was provided through R01AG023629 from the National Institute on Aging (NIA). A full list of principal CHS investigators and institutions can be found at CHS-NHLBI.org. The provision of genotyping data was supported in part by the National Center for Advancing Translational Sciences, CTSI grant UL1TR001881, and the National Institute of Diabetes and Digestive and Kidney Disease Diabetes Research Center (DRC) grant DK063491 to the Southern California Diabetes Endocrinology Research Center. The content is solely the responsibility of the authors and does not 
COPSAC2000

CROATIA_Korcula

DIABNORD

\section{DPS}

necessarily represent the official views of the National Institutes of Health.

We greatly acknowledge the private and public research funding allocated to COPSAC and listed on www.copsac.com, with special thanks to The Lundbeck Foundation (Grant nr. R16-A1694); Ministry of Health (Grant nr. 903516); Danish Council for Strategic Research (Grant nr.: 0603-00280B); The Danish Council for Independent Research and The Capital Region Research Foundation as core supporters. The funding agencies did not have any influence on study design, data collection and analysis, decision to publish or preparation of the manuscript. No pharmaceutical company was involved in the study. We gratefully express our gratitude to the participants of the COPSAC2000 cohort study for all their support and commitment. We also acknowledge and appreciate the unique efforts of the COPSAC research team.

We would like to acknowledge the contributions of the recruitment team in Korcula, the administrative teams in Croatia and Edinburgh and the people of Korcula. Exome array genotyping was performed at the Clinical Research Facility University of Edinburgh, Edinburgh, UK and funded by UK's Medical Research Council

We are grateful to the study participants who dedicated their time and samples to these studies. We also thank the VHS, the Swedish Diabetes Registry and Umeå Medical Biobank staff for biomedical data and DNA extraction. We also thank $M$ Sterner, G Gremsperger and P Storm for their expert technical assistance with genotyping and genotype data preparation. The current study was funded by Novo Nordisk, the Swedish Research Council, Påhlssons Foundation, the Swedish Heart Lung Foundation, and the Skåne Regional Health Authority (all to PWF).

The DPS has been financially supported by grants from the Academy of Finland (117844 and 40758, 211497, and 118590 (MU); The EVO funding of the Kuopio University Hospital from Ministry of Health and Social Affairs (5254), Finnish Funding Agency for Technology and Innovation (40058/07), Nordic Centre of Excellence on 'Systems biology in controlled dietary interventions and cohort studies, SYSDIET (070014), The Finnish Diabetes Research Foundation, Yrjö Jahnsson Foundation (56358), Sigrid Juselius Foundation and TEKES grants 70103/06 and 40058/07. 
DR'S EXTRA

Edmonton Human islet data

$\mathrm{EFSOCH}$

EPIC-Norfolk

EPIC-Potsdam
The DR's EXTRA Study was supported by the Ministry of Education and Culture of Finland (722 and 627;2004-2011), Academy of Finland (102318; 104943;123885;

211119), Kuopio University Hospital, Finnish Diabetes Association, Finnish Foundations for Cardiovascular Research, Päivikki and Sakari Sohlberg Foundation, by European Commission FP6 Integrated Project (EXGENESIS); LSHM-CT-2004005272, City of Kuopio and Social Insurance Institution of Finland (4/26/2010). The authors thank James Lyon at the Alberta Diabetes Institute IsletCore for his work on human islet isolations. We also thank the Human Organ Procurement and Exchange Program (Edmonton) and the Trillium Gift of Life Network (Toronto) and other organ procurement agencies for their efforts in obtaining human pancreas for research.

This paper presents independent research supported by the National Institute for Health Research (NIHR) Exeter Clinical Research Facility. The views expressed are those of the author(s) and not necessarily those of the NHS, the NIHR or the Department of Health.

The EPIC-Norfolk study (DOI 10.22025/2019.10.105.00004) has received funding from the Medical Research Council (MR/N003284/1 and MC-UU_12015/1) and Cancer Research UK (C864/A14136). The genetics work in the EPIC-Norfolk study was funded by the Medical Research Council ((MC_PC_13048)).We are grateful to all the participants who have been part of the project and to the many members of the study teams at the University of Cambridge who have enabled this research The study was supported in part by a grant from the German Federal Ministry of Education and Research (BMBF) to the German Center for Diabetes Research (DZD e.V.). The recruitment phase of the EPIC-Potsdam study was supported by the Federal Ministry of Science, Germany (01 EA 9401) and the European Union (SOC 9520140805 F02). The follow-up of the EPIC-Potsdam study was supported by German Cancer Aid (70-2488-Ha I) and the European Community (SOC 98200769 05 F02). Exome chip genotyping of EPIC-Potsdam samples was carried out under supervision of Per Hoffmann and Stefan Herms at Life \& Brain GmbH, Bonn. We are grateful to the Human Study Centre (HSC) of the German Institute of Human Nutrition Potsdam-Rehbrücke, namely the trustee and the data hub for the processing, and the participants for the provision of the data, the biobank for the 
EpiHealth

ERF STUDY processing of the biological samples and the head of the HSC, Manuela Bergmann, for the contribution to the study design and leading the underlying processes of data generation.

EpiHealth was supported by the Swedish Research Council strategic research network Epidemiology for Health, Uppsala University and Lund University. Genotyping in EpiHealth was supported by Swedish Heart-Lung Foundation (grant no. 20120197 and 20140422), Knut och Alice Wallenberg Foundation (grant no. 2013.0126), and Swedish Research Council (grant no. 2012-1397). Genotyping was performed by the SNP\&SEQ Technology Platform in Uppsala. We thank the EpiHealth participants for their dedication and commitment.

ERF study is grateful to all study participants and their relatives, general practitioners and neurologists for their contributions and to $P$. Veraart for her help in genealogy, J. Vergeer for the supervision of the laboratory work and P. Snijders for his help in data collection.

Funding: Erasmus Rucphen Family (ERF) was supported by the Consortium for Systems Biology (NCSB), both within the framework of the Netherlands Genomics Initiative (NGI)/Netherlands Organisation for Scientific Research (NWO). ERF study as a part of EUROSPAN (European Special Populations Research Network) was supported by European Commission FP6 STRP grant number 018947 (LSHG-CT2006-01947) and also received funding from the European Community's Seventh Framework Programme (FP7/2007-2013)/grant agreement HEALTH-F4-2007201413 by the European Commission under the programme "Quality of Life and Management of the Living Resources" of 5th Framework Programme (no. QLG2CT-2002-01254) as well as FP7 project EUROHEADPAIN (nr 602633). Highthroughput analysis of the ERF data was supported by joint grant from Netherlands Organisation for Scientific Research and the Russian Foundation for Basic Research (NWO-RFBR 047.017.043).The exome-chip measurements have been funded by the Netherlands Organization for Scientific Research (NWO; project number 184021007) and by the Rainbow Project (RP10; Netherlands Exome Chip Project) of the Biobanking and Biomolecular Research Infrastructure Netherlands (BBMRI-NL; www.bbmri.nl (http://www.bbmri.nl) ). Ayse Demirkan is supported by a Veni grant (2015) from ZonMw. Ayse Demirkan, Jun Liu and 
Family Heart Study

(FamHS)

Fenland

FIA3

FIN-D2D 2007

FINRISK 2007

Framingham Heart

Study
Cornelia van Duijn have used exchange grants from PRECEDI. The funders had no role in study design, data collection and analysis, decision to publish, or preparation of the manuscripts.

The Family Heart Study (FamHS) was supported by NIH grants R01-HL-087700 and R01-HL-088215 from NHLBI, and R01-DK-8925601 and R01-DK-075681 from NIDDK.

The Fenland Study is funded by the Medical Research Council (MC_U106179471) and Wellcome Trust. We are grateful to all the volunteers for their time and help, and to the General Practitioners and practice staff for assistance with recruitment. We thank the Fenland Study Investigators, Fenland Study Co-ordination team and the Epidemiology Field, Data and Laboratory teams.

Jansson J-H was responsible for the identification of MI cases. The FIA3 study was supported in part by a grant from the Swedish Heart-Lund Foundation (to PWF). The FIN-D2D 2007 study was supported by funds from the hospital districts of Pirkanmaa; Southern Ostrobothnia; North Ostrobothnia; Central Finland and Northern Savo; the Finnish National Public Health Institute; the Finnish Diabetes Association; the Ministry of Social Affairs and Health in Finland; Finland's Slottery Machine Association; the Academy of Finland [grant number 129293] and Commission of the European Communities, Directorate C-Public Health [grant agreement no. 2004310].

VS was supported by the Finnish Foundation for Cardiovascular Research. PJ was supported by the Academy of Finland \#118065.

FHS: Genotyping, quality control and calling of the Illumina HumanExome BeadChip in the Framingham Heart Study was supported by funding from the National Heart, Lung and Blood Institute Division of Intramural Research (Daniel Levy and Christopher J. O'Donnell, Principle Investigators). Also supported by National Institute for Diabetes and Digestive and Kidney Diseases (NIDDK) U01 DK078616, NIDDK K24 DK080140 and American Diabetes Association MentorBased Postdoctoral Fellowship Award \#7-09-MN-32, all to Dr. Meigs, and NIDDK Research Career Award K23 DK65978, a Massachusetts General Hospital Physician Scientist Development Award and a Doris Duke Charitable Foundation Clinical Scientist Development Award to Dr. Florez. 
FUSION

Generation

Scotland

GENOA

GIANT

GLACIER

GoDARTS
The FUSION study was supported by DK093757, DK072193, DK062370, and ZIAHG000024. HAK has received funding from Academy of Finland (support for clinical research careers, grant no 258753).

We would like to acknowledge the contributions of the families who took part in the Generation Scotland: Scottish Family Health Study, the general practitioners and Scottish School of Primary Care for their help in recruiting them, and the whole Generation Scotland team, which includes academic researchers, IT staff, laboratory technicians, statisticians and research managers. Generation Scotland received core funding from the Chief Scientist Office of the Scottish Government Health Directorate CZD/16/6 and the Scottish Funding Council HR03006.

Genotyping of the GS:SFHS samples was carried out by staff at the Genetics Core Laboratory at the Clinical Research Facility, University of Edinburgh, Scotland and was funded by the UK's Medical Research Council.

Support for GENOA was provided by the National Heart, Lung and Blood Institute (HL054464; HL054481; HL087660; HL119443; HL086694) of the National Institutes of Health. We thank Eric Boerwinkle, PhD and Megan L. Grove, MS, University of Texas Health Science Center, Houston, Texas, USA for their help with genotype calling. We would also like to thank the families that participated in the GENOA study.

Anne E Justice (AEJ) is funded under NIH 5K99HL130580-02.

We are indebted to the study participants who dedicated their time and samples to these studies. We J Hutiainen and Å Ågren (Umeå Medical Biobank) for data organization and K Enquist and T Johansson (Västerbottens County Council) for technical assistance with DNA extraction. We also thank M Sterner, G Gremsperger and P Storm for their expert technical assistance with genotyping and genotype data preparation. The current study was funded by Novo Nordisk, the Swedish Research Council, Påhlssons Foundation, the Swedish Heart Lung Foundation, and the Skåne Regional Health Authority (all to PWF).

GoDARTS study was funded by The Wellcome Trust Study Cohort Wellcome Trust Functional Genomics Grant (2004-2008) (Grant No: 072960/2/03/2) and The Wellcome Trust Scottish Health Informatics Programme (SHIP) (2009-2012). (Grant No: 086113/Z/08/Z). 
$\mathrm{HABC}$

HANDLS (Healthy

Aging in

Neighborhoods of

Diversity across

the Life Span

study)

Health and

Retirement Study

(HRS)

Health2006

Health2008

Heather M.

Highland
The Health, Aging, and Body Composition (HABC) Study is supported by NIA contracts N01AG62101, N01AG62103, and N01AG62106. The genome-wide association study was funded by NIA grant 1R01AG032098-01A1 to Wake Forest University Health Sciences.

We would like to thank the Healthy Aging in Neighborhoods of Diversity across the Life Span (HANDLS) study participants, study coordinator, medical staff and field workers. Exome chip genotyping was performed at the Laboratory of Neurogenetics, National Institute on Aging, National Institutes of Health (NIH). The HANDLS study was supported by the Intramural Research Program of the NIH, National Institute on Aging and the National Center on Minority Health and Health Disparities (project \# Z01-AG000513 and human subjects protocol number 09-AGN248). Data analyses for the HANDLS study utilized the computational resources of the NIH HPC Biowulf cluster at the National Institutes of Health, Bethesda, MD. (http://hpc.nih.gov).

HRS is supported by the National Institute on Aging (NIA U01AG009740). The genotyping was funded separately by the National Institute on Aging (RC2 AG036495, RC4 AG039029). Our genotyping was conducted by the NIH Center for Inherited Disease Research (CIDR) at Johns Hopkins University. Genotyping quality control and final preparation of the data were performed by the University of Michigan School of Public Health.

The Health2006 was financially supported by grants from the Velux Foundation; The Danish Medical Research Council, Danish Agency for Science, Technology and Innovation; The Aase and Ejner Danielsens Foundation; ALK-Abello A/S, Hørsholm, Denmark, and Research Centre for Prevention and Health, the Capital Region of Denmark.

The Health2008 was supported by the Timber Merchant Vilhelm Bang's Foundation, the Danish Heart Foundation (Grant number 07-10-R61-A1754-B83822392F), and the Health Insurance Foundation (Helsefonden) (Grant number 2012B233).

Heather M. Highland is supported by funding from NHLBI training grant T32 HL007055. 
HELIC MANOLIS and HELIC Pomak

Hidetoshi Kitajima

Inês Barroso Inter99

InterAct

Consortium

Jennifer Asimit
This work was funded by the Wellcome Trust (098051) and the European Research Council (ERC-2011-StG 280559-SEPI). The MANOLIS cohort is named in honour of Manolis Giannakakis, 1978-2010. We thank the residents of the Mylopotamos villages, and of the Pomak villages, for taking part. The HELIC study has been supported by many individuals who have contributed to sample collection (including A. Athanasiadis, O. Balafouti, C. Batzaki, G. Daskalakis, E. Emmanouil, C. Giannakaki, M. Giannakopoulou, A. Kaparou, V. Kariakli, S. Koinaki, D. Kokori, M. Konidari, H. Koundouraki, D. Koutoukidis, V. Mamakou, E. Mamalaki, E. Mpamiaki, M. Tsoukana, D. Tzakou, K. Vosdogianni, N. Xenaki, E. Zengini), data entry (T. Antonos, D. Papagrigoriou, B. Spiliopoulou), sample logistics (S. Edkins, E. Gray), genotyping (R. Andrews, H. Blackburn, D. Simpkin, S. Whitehead), research administration (A. Kolb-Kokocinski, S. Smee, D. Walker) and informatics (M. Pollard, J. Randall).

Hidetoshi Kitajima was funded by Manpei Suzuki Diabetes Foundation Grant-in-Aid for the young scientists working abroad.

Inês Barroso is funded by Wellcome (WT206194)

The Inter99 was initiated by Torben Jørgensen (PI), Knut Borch-Johnsen (co-PI), Hans Ibsen and Troels F. Thomsen. The steering committee comprises the former two and Charlotta Pisinger. The study was financially supported by research grants from the Danish Research Council, the Danish Centre for Health Technology Assessment, Novo Nordisk Inc., Research Foundation of Copenhagen County, Ministry of Internal Affairs and Health, the Danish Heart Foundation, the Danish Pharmaceutical Association, the Augustinus Foundation, the Ib Henriksen Foundation, the Becket Foundation, and the Danish Diabetes Association. Funding for the InterAct project was provided by the EU FP6 programme (grant number LSHM_CT_2006_037197). We thank all EPIC participants and staff for their contribution to the study. We thank the lab team at the MRC Epidemiology Unit for sample management and Nicola Kerrison for data management. Medical Research Council Methodology Research Fellowship (MR/K021486/1) 
The JHS is supported by contracts HHSN268201300046C, HHSN268201300047C, HHSN268201300048C, HHSN268201300049C, HHSN268201300050C from the National Heart, Lung and Blood Institute and the National Institute on Minority Health and Health Disparities. ExomeChip genotyping was supported by the NHLBI of the National Institutes of Health under award number R01HL107816 to S. Kathiresan. The content is solely the responsibility of the authors and does not necessarily represent the official views of the National Institutes of Health.

Joel N. Hirschhorn

KORA

Leipzig Adults
NIH R01DK075787

The KORA study was initiated and financed by the Helmholtz Zentrum München German Research Center for Environmental Health, which is funded by the German Federal Ministry of Education and Research (BMBF) and by the State of Bavaria. Furthermore, KORA research was financed by a grant from the BMBF to the German Center for Diabetes Research (DZD) and a grant from the Ministry of Innovation, Science, Research and Technology of the state North RhineWestphalia (Düsseldorf, Germany). It was also supported within the Munich Center of Health Sciences (MC-Health), Ludwig-Maximilians-Universität, as part of LMUinnovativ. The authors are grateful to all members of the Helmholtz Zentrum München, the field staff in Augsburg, and the Augsburg registry team who were involved in the planning, organization, and conduct of the KORA studies. In addition, the authors express their appreciation to all study participants. This work was supported by grants from the German Research Council (SFB- 1052 "Obesity mechanisms"; B01; B03), from the German Diabetes Association and from the DHFD (Diabetes Hilfs- und Forschungsfonds Deutschland). IFB AdiposityDiseases is supported by the Federal Ministry of Education and Research (BMBF), Germany, FKZ: 01EO1501 (AD2-060E, AD2-06E99). This work was further supported by the Kompetenznetz Adipositas (Competence network for Obesity) funded by the Federal Ministry of Education and Research (German Obesity Biomaterial Bank; FKZ 01GI1128). 
Leipzig-Childhood- This work was supported by grants from Integrated Research and Treatment IFB Centre (IFB) Adiposity Diseases, from the German Research Foundation for the Clinical Research Group "Atherobesity" KFO 152 (KO3512/1 to AK), and by the European Commission (Beta-JUDO) and by EFRE (LIFE Child Obesity). We are grateful to all the patients and families for contributing to the study. We highly appreciate the support of the Obesity Team and Auxo Team of the Leipzig University Children's Hospital for management of the patients and to the Pediatric Research Center Lab Team for support with DNA banking.

LOLIPOP (Exome, The LOLIPOP study is supported by the National Institute for Health Research OmniEE) (NIHR) Comprehensive Biomedical Research Centre Imperial College Healthcare NHS Trust, the British Heart Foundation (SP/04/002), the Medical Research Council (G0601966,G0700931), the Wellcome Trust (084723/Z/08/Z), the NIHR (RP-PG0407-10371), European Union FP7 (EpiMigrant, 279143) and Action on Hearing Loss (G51). We thank the participants and research staff who made the study possible.

Lothian Birth We thank the cohort participants and team members who contributed to these Cohort 1921 and studies. Phenotype collection in the Lothian Birth Cohort 1921 was supported by Lothian Birth Cohort 1936 the UK's Biotechnology and Biological Sciences Research Council (BBSRC), The Royal Society and The Chief Scientist Office of the Scottish Government. Phenotype collection in the Lothian Birth Cohort 1936 was supported by Age UK (The Disconnected Mind project). Genotyping was supported by Centre for Cognitive Ageing and Cognitive Epidemiology (Pilot Fund award), Age UK, and the Royal Society of Edinburgh. The work was undertaken by The University of Edinburgh Centre for Cognitive Ageing and Cognitive Epidemiology, part of the cross council Lifelong Health and Wellbeing Initiative (MR/K026992/1). Funding from the BBSRC and Medical Research Council (MRC) is gratefully acknowledged. Mark I McCarthy is a Wellcome Trust Senior Investigator (WT098381); and a National Institute of Health Research Senior Investigator. 
MESA

METSIM

$\mathrm{MvdB}$

Natasha H J Ng

NEO
MESA and the MESA SHARe project are conducted and supported by the National Heart, Lung, and Blood Institute (NHLBI) in collaboration with MESA investigators.

Support for MESA is provided by contracts HHSN268201500003I, N01-HC-95159, N01-HC-95160, N01-HC-95161, N01-HC-95162, N01-HC-95163, N01-HC-95164, N01-HC-95165, N01-HC-95166, N01-HC-95167, N01-HC-95168, N01-HC-95169, UL1-TR-000040, UL1-TR-001079, UL1-TR-001420. The provision of genotyping data was supported in part by the National Center for Advancing Translational Sciences, CTSI grant UL1TR001881, and the National Institute of Diabetes and Digestive and Kidney Disease Diabetes Research Center (DRC) grant DK063491 to the Southern California Diabetes Endocrinology Research Center.

The METSIM study was supported by the Academy of Finland (contract 124243), the Finnish Heart Foundation, the Finnish Diabetes Foundation, Tekes (contract 1510/31/06), and the Commission of the European Community (HEALTH-F2-2007 201681), and the US National Institutes of Health grants DK093757, DK072193, DK062370, and ZIA- HG000024.

MvdB is supported by a Novo Nordisk postdoctoral fellowship run in partnership with the University of Oxford Natasha H J Ng (NHJN) is supported by the National Science Scholarship from the Agency for Science, Technology and Research (A*STAR) in Singapore.

The authors of the NEO study thank all individuals who participated in the Netherlands Epidemiology of Obesity study, all participating general practitioners for inviting eligible participants and all research nurses for collection of the data. We thank the NEO study group, Pat van Beelen, Petra Noordijk and Ingeborg de Jonge for the coordination, lab and data management of the NEO study. The genotyping in the NEO study was supported by the Centre National de Génotypage (Paris, France), headed by Jean-Francois Deleuze. The NEO study is supported by the participating Departments, the Division and the Board of Directors of the Leiden University Medical Center, and by the Leiden University, Research Profile Area Vascular and Regenerative Medicine. Dennis MookKanamori is supported by Dutch Science Organization (ZonMW-VENI Grant 916.14.023). 
Nicola L. Beer

NFBC66 and

NFBC86

OBB

PIVUS \& ULSAM
NLB was a Naomi Berrie Fellow in Diabetes Research. NLB is now an employee of Novo Nordisk, although all experimental work was carried out under employment at the University of Oxford.

NFBC1966 and 1986 received financial support from the Academy of Finland (project grants 104781, 120315, 129269, 1114194, 24300796, Center of Excellence in Complex Disease Genetics and SALVE), University Hospital Oulu, Biocenter, University of Oulu, Finland (75617), NIHM (MH063706, Smalley and Jarvelin), Juselius Foundation, NHLBI grant 5R01HL087679-02 through the STAMPEED program (1RL1MH083268-01), NIH/NIMH (5R01MH63706:02), the European Commission (EURO-BLCS, Framework 5 award QLG1-CT-2000-01643), ENGAGE project and grant agreement HEALTH-F4-2007-201413, EU FP7 EurHEALTHAgeing -277849, the Medical Research Council, UK (G0500539, G0600705, G1002319, PrevMetSyn/SALVE) and the MRC, Centenary Early Career Award. The program is currently being funded by the H2020-633595 DynaHEALTH action and academy of Finland EGEA-project (285547). The DNA extractions, sample quality controls, biobank up-keeping and aliquotting was performed in the National Public Health Institute, Biomedicum Helsinki, Finland and supported financially by the Academy of Finland and Biocentrum Helsinki. We thank the late Professor Paula Rantakallio (launch of NFBCs), and Ms. Outi Tornwall and Ms. Minttu Jussila (DNA biobanking). The authors would like to acknowledge the contribution of the late Academian of Science Leena Peltonen.

The Oxford Biobank is supported by the Oxford Biomedical Research Centre and part of the National NIHR Bioresource.

PIVUS and ULSAM are supported by the Swedish Research Council, Swedish HeartLung Foundation, Swedish Diabetes Foundation and Uppsala University. The investigators express their deepest gratitude to the study participants.

Genotyping and analysis was funded by the Wellcome Trust under awards WT064890, WT090532 and WT098017. 
PPP-Botnia

PROMIS

Robert Sladek

Rebecca Fine

RISC
The Botnia and The PPP-Botnia studies (L.G., T.T.) have been financially supported by grants from Folkhälsan Research Foundation, the Sigrid Juselius Foundation, The Academy of Finland (grants no. 263401, 267882, 312063 to LG, 312072 to TT), Nordic Center of Excellence in Disease Genetics, EU (EXGENESIS, MOSAIC FP7600914), Ollqvist Foundation, Swedish Cultural Foundation in Finland, Finnish Diabetes Research Foundation, Foundation for Life and Health in Finland, Signe and Ane Gyllenberg Foundation, Finnish Medical Society, Paavo Nurmi Foundation, Helsinki University Central Hospital Research Foundation, Perklén Foundation, Närpes Health Care Foundation and Ahokas Foundation. The study has also been supported by the Ministry of Education in Finland, Municipal Heath Care Center and Hospital in Jakobstad and Health Care Centers in Vasa, Närpes and Korsholm. The research leading to these results has received funding from the European Research Council under the European Union's Seventh Framework Programme (FP7/2007-2013) / ERC grant agreement $n^{\circ} 269045$. The skillful assistance of the Botnia Study Group is gratefully acknowledged.

Dr. Saleheen has received grants from the National Heart, Lung and Blood Institute, Fogarty International, Pfizer, Regeneron, Eli Lilly, and Genentech. RS is the recipient of a Chercheur Boursier award from the Fonds de la Recherche en Santé du Québec and a New Investigator Award from the Canadian Institutes of Health Research; and is supported by operating funds from the Canadian Institutes of Health Research.

Rebecca Fine is supported by NHGRI F31 HG009850.

The RISC study was supported by European Union grant QLG1-CT-2001-01252 and AstraZeneca. The initial genotyping of the RISC samples was funded by Merck \& Co Inc. 
Rotterdam study The Rotterdam Study is funded by Erasmus Medical Center and Erasmus University, Rotterdam, Netherlands Organization for the Health Research and Development (ZonMw), the Research Institute for Diseases in the Elderly (RIDE), the Ministry of Education, Culture and Science, the Ministry for Health, Welfare and Sports, the European Commission (DG XII), and the Municipality of Rotterdam. The authors are grateful to the study participants, the staff from the Rotterdam Study and the participating general practitioners and pharmacists. The generation and management of the Illumina exome chip v1.0 array data for the Rotterdam Study (RS-I) was executed by the Human Genotyping Facility of the Genetic Laboratory of the Department of Internal Medicine, Erasmus MC, Rotterdam, The Netherlands. The Exome chip array data set was funded by the Genetic Laboratory of the Department of Internal Medicine, Erasmus MC, from the Netherlands Genomics Initiative (NGI)/Netherlands Organisation for Scientific Research (NWO)sponsored Netherlands Consortium for Healthy Aging (NCHA; project nr. 050-060810); the Netherlands Organization for Scientific Research (NWO; project number 184021007) and by the Rainbow Project (RP10; Netherlands Exome Chip Project) of the Biobanking and Biomolecular Research Infrastructure Netherlands (BBMRINL; www.bbmri.nl (http://www.bbmri.nl)). We thank Ms. Mila Jhamai, Ms. Sarah Higgins, and Mr. Marijn Verkerk for their help in creating the exome chip database, and Carolina Medina-Gomez, Lennart Karssen, and Linda Broer for QC and variant calling. We are grateful to the study participants, the staff from the Rotterdam Study and the participating general practitioners and pharmacists. $\mathrm{Dr}$ Franco is employed by ErasmusAGE, a center for aging research across the life course funded by Nestlé Nutrition (Nestec Ltd.) and Metagenics.

SardiNIA The SardiNIA investigators thank all the volunteers who generously participated in this study and made this research possible. The study is supported by National Human Genome Research Institute grants HG005581, HG005552, HG006513, HG007022 and HG007089; by National Heart, Lung, and Blood Institute grant HL117626; by the Intramural Research Program of the US National Institutes of Health, National Institute on Aging, contracts N01-AG-1-2109 and HHSN271201100005C; by Sardinian Autonomous Region (L.R. 7/2009) grant cRP3154 
The Singapore

Chinese Eye Study

(SCES)

Sorbs

Timothy D Spector

TwinsUK

UKHLS

Vejle Biobank
The Singapore Chinese Eye Study was funded by the Agency for Science, Technology and Research - Biomedical Research Council (A*STAR BMRC) Grant, Singapore [08/1/35/19/550] and Singapore Ministry of Health's National Medical Research Council [NMRC/CIRG/1417/2015]. The authors gratefully acknowledge use of the services and facilities of the Singapore Eye Research Institute and Singapore National Eye Centre. We also acknowledge the contributions of all participants who volunteered and the personnel responsible for the recruitment and administration of the study.

We thank all those who participated in the study. We would like to thank Knut Krohn (Microarray Core Facility, University of Leipzig, Institute of Pharmacology) for the genotyping support and Joachim Thiery (Institute of Laboratory Medicine, Clinical Chemistry and Molecular Diagnostics, University of Leipzig) for clinical chemistry services. This work was supported by grants from the German Research Council (DFG - SFB 1052 “Obesity mechanisms”; A01, C01, B03 and SPP 1629 TO 718/2-1), from the German Diabetes Association and from the DHFD (Diabetes Hilfs- und Forschungsfonds Deutschland).

Timothy D Spector is holder of an ERC Advanced Principal Investigator award. TwinsUK study was funded by the Wellcome Trust; European Community's Seventh Framework Programme (FP7/2007-2013). The study also receives support from the National Institute for Health Research (NIHR) BioResource Clinical Research Facility and Biomedical Research Centre based at Guy's and St Thomas' NHS Foundation Trust and King's College London.

These data are from Understanding Society: The UK Household Longitudinal Study, which is led by the Institute for Social and Economic Research at the University of Essex and funded by the Economic and Social Research Council. The data were collected by NatCen and the genome wide scan data were analysed by the Wellcome Trust Sanger Institute. The Understanding Society DAC have an application system for genetics data and all use of the data should be approved by them. The application form is at: https://www.understandingsociety.ac.uk/about/health/data. The Vejle Diabetes Biobank was supported by The Danish Research Council for Independent Research. 
WGHS

The WGHS is funded by the National Heart, Lung, and Blood Institute (HL043851 and HL080467) and National Cancer Institute (CA047988 and UM1CA182913).

Funding for genotyping on the Exome Array was funded by Amgen.

1019 Disclosures

1020

1021 Soren K. Thomsen: SKT is now an employee of Vertex Pharmaceuticals, although all experimental work 1022 was carried out under employment at the University of Oxford.

1023 Martijn van de Bunt: Currently employed by Novo Nordisk.

1024 Audrey Y Chu: Currently employed by Merck.

1025 Dennis O. Mook-Kanamori: Dennis Mook-Kanamori is working as a part-time clinical research consultant 1026 for Metabolon, Inc.

1027 Paul W. Franks: PWF has been a paid consultant for Eli Lilly and Sanofi Aventis and has received research 1028 support from several pharmaceutical companies as part of a European Union Innovative Medicines 1029 Initiative (IMI) project.

1030 Mike A. Nalls: Dr. Mike A. Nalls is supported by a consulting contract between Data Tecnica International 1031 LLC and the National Institute on Aging (NIA), National Institutes of Health (NIH), Bethesda, MD, USA. Dr. 1032 Nalls also consults for Illumina Inc., the Michael J. Fox Foundation, and the University of California 1033 Healthcare.

1034 Mark J. Caulfield: MJC is Chief Scientist for Genomics England, a UK government company.

1035 Joel N. Hirschhorn: JHN is on the scientific advisory board of Camp4 Therapeutics.

1036 Erik Ingelsson: Erik Ingelsson is an advisor and consultant for Precision Wellness, Inc., and advisor for 1037 Cellink.

1038 Inês Barroso: IB and spouse declare stock ownership in GlaxoSmithkline and Incyte Ltd. 


\section{Supplementary Figure Legends}

Figure S1. GeneMANIA network analysis identifies relevant pathways regulating glycemia. The networks represent composite networks for (A) FI and (B) $2 \mathrm{hGlu}$, from the GeneMANIA analysis using genes with variant associations at $P<1 \times 10^{-5}$ for each trait as input. Nodes outlined in red correspond to genes from the input list. Other nodes correspond to related genes based on 50 default databases.

Based on the network, GO terms and Reactome pathways that were significantly enriched are depicted. To summarize these results, the most significant term of all calculated terms within the same group (using the Kappa method, see Methods) was represented. Each group was assigned a specific color; if a gene is present in more than one term, it will be displayed in more than one color. Barplots with the Bonferroni-adjusted -log10(p-values) of the most significant terms within each group are are shown. Each group was assigned a specific color; if a gene is present in more than one term, it is displayed in more than one color. Details of the networks are summarized in (C). Related to Figure 2 and Table S7.

Figure S2. Pathway analysis identifies relevant gene sets regulating glycemia. EC-DEPICT analysis with heatmap visualization (UK Biobank permutations) is shown for a. all traits combined; b. HbA1c only; c. all traits except HbA1c combined; d. FG only; e. 2 hGlu only. The columns represent the input genes for the analysis. We used affinity propagation clustering to define a representative "meta-gene set" for groups of highly correlated gene sets (see Methods); the rows in the heat map represent significant meta-gene sets (FDR <0.05). The color of each square indicates DEPICT's z-score for membership of that gene in that gene set, where dark red means "very likely a member" and dark blue means "very unlikely a member". The gene set annotations indicate whether that meta-gene set was significant at FDR $<0.05$ or not significant (n.s.) for each of the other EC-DEPICT analyses using the UK Biobank permutations (all traits together, HbA1c only, FG only, 2hGlu only, and all-except-HbA1c). For heatmap intensity and ECDEPICT $P$-values, the meta-gene set values are taken from the most significantly enriched member gene set. The gene variant annotations are as follows: (1) the European minor allele frequency (MAF) of the input variant, where rare is $M A F<1 \%$, low-frequency is MAF $1-5 \%$, and common is $\operatorname{MAF}>5 \%$, (2) whether the gene has an Online Mendelian Inheritance in Man (OMIM) annotation as causal for a diabetes/glycemic-relevant syndrome or blood disorder, (3) the effector transcript classification for that variant: gold, silver, bronze, or NA (note that only array-wide significant variants were classified, so suggestively-significant variants are by default classified as "NA"), (4-7) whether each variant was 
1070

1071

1072

1073

1074

1075

1076

1077

1078

1079

1080

1081

1082

1083

1084

1085

1086

1087

1088

1089

1090

1091

1092

1093

1094

1095

1096

1097

1098

1099

significant $\left(P<2 \times 10^{-7}\right)$, suggestively significant $\left(P<10^{-5}\right)$, or not significant in Europeans for each of the four traits, and (8) whether each variant was classified in the analysis (with UK Biobank permutations) or excluded by filters (see Methods). AWS: array-wide significant. Related to Figure 2 and Table S8.

Figure S3. Pathway analysis identifies relevant gene sets regulating glycemia. EC-DEPICT analysis with heatmap visualization (Swedish permutations) is shown for a. all traits combined; b. HbA1c only; c. all traits except $\mathrm{HbA1c}$ combined; $d$. FG only. (With these permutations, there was no significance for $2 \mathrm{hGlu}$ only). We used affinity propagation clustering to define a representative "meta-gene set" for groups of highly-correlated gene sets (see Methods); the rows in the heat map represent significant meta-gene sets (FDR <0.05). The color of each square indicates DEPICT's z-score for membership of that gene in that gene set, where dark red means "very likely a member" and dark blue means "very unlikely a member". The gene set annotations indicate whether that meta-gene set was significant at FDR $<0.05$ or not significant (n.s.) for each of the other EC-DEPICT analyses using the Swedish permutations (all traits together, HbA1c only, FG only, and all-except-HbA1c). For heatmap intensity and EC-DEPICT $P$-values, the meta-gene set values are taken from the most significantly enriched member gene set. The gene variant annotations are as follows: (1) the European minor allele frequency (MAF) of the input variant, where rare is $\mathrm{MAF}<1 \%$, low-frequency is $\mathrm{MAF} 1-5 \%$, and common is $M A F>5 \%$, (2) whether the gene has an Online Mendelian Inheritance in Man (OMIM) annotation as causal for a diabetes/glycemic-relevant syndrome or blood disorder, (3) the effector transcript classification for that variant: gold, silver, bronze, or NA (note that only array-wide significant variants were classified, so suggestively-significant variants are by default classified as "NA"), (4-7) whether each variant was significant $\left(P<2 \times 10^{-7}\right)$, suggestively significant $\left(P<10^{-5}\right)$, or not significant in Europeans for each of the four traits, and (8) whether each variant was included in the analysis (with Swedish permutations) or excluded by filters (see Methods). AWS: array-wide significant. Related to Figure 2 and Table S8.

\section{Figure S4. Functional characterisation of G6PC variants. Related to Figure 4.}

(A) Cellular localisation of Q347X was assessed in HEK293 cells and overlaid with a marker for the ER, calreticulin, (left) or the trans-golgi network, TGN46 (right). White arrows point to positions of the golgi apparatus. Scale bar indicates 10 $\mathrm{m}$. (B) Glucose-6-phosphatase activity of G6PC-R83C (n=3), with representative western blot of microsomal protein isolated from HEK293 shown. (C) Glucose-6- 
1100 phosphatase activity of G6PC-Q347X $(n=2)$, with representative western blot of microsomal protein

1101 isolated from HEK293 shown. (D) Protein expression levels of G6PC-A204S in microsomal protein

1102 extracted from HEK293 cells was found to be downregulated by $41 \%$ compared to WT based on 1103 densitometric analysis $(n=4)$, with representative western blot shown. Data presented as mean \pm SEM 1104 and analysed using paired Students' t test. * $p=0.01$. Unt: Untransfected; WT: Wild type.

Figure S5. Functional characterisation of G6PC2 variants and the effect of G6PC2 knockdown on insulin content and secretion in EndoC- $\beta \mathrm{H} 1$ cells. Related to Figure 5.

1108 (A) Variants prioritised for functional study in the context of the predicted G6PC2 protein structure 1109 (RefSeq NP_066999.1) in the ER membrane. Amino acid residues are coloured as described in the 1110 legend. Variants selected for functional study, in green, are labelled. The N-terminal V5 and C-terminal 1111 Myc-FLAG tags present in the expression constructs are indicated. (B) Quantification of total G6PC2 1112 variant protein expression (both upper and lower bands of representative western blot in Figure 5) in 1113 INS-1 832/13 cells based on western blot densitometric analysis of Myc-tagged G6PC2 constructs 1114 relative to tubulin control $(n=5)$. (C) Expression levels of G6PC2 variant proteins in HEK293 by western 1115 blot densitometric analysis of FLAG-tagged G6PC2 constructs or V5-tagged G6PC2-R283X relative to 1116 tubulin control $(n=4)$. Representative blots are shown for untreated cells and cells treated with 1117 proteasomal inhibitor MG-132 or lysosomal inhibitor chloroquine. (D) Glucose-6-phosphatase activity of 1118 the R281X variant in G6PC (proxy for R283X in G6PC2) in HEK293 ( $n=2$ ), with representative western blot 1119 of microsomal protein shown. (E) Total insulin secretion and insulin content were assessed at basal and 1120 high glucose conditions (with and without drug treatment) following 96-120h G6PC2 knockdown in 1121 EndoC- $\beta$ H1. Unpaired two-tailed Students' t tests were used to compare G6PC2 knockdown to control 1122 for each condition, from $n=16$ across 4 independent experiments. Tol: tolbutamide; Diaz: diazoxide. All 1123 data presented as mean \pm SEM. * $p=0.01-0.05 ; * * p=0.001-0.01 ; * * p<0.001$.

Figure S6. G6PC2 expression in RNA-Seq data from 150 human islet donor samples. (A) Allelic balance

1126 was observed for G6PC2 rs146779637 (p.R283X) in two heterozygote human islet samples. (B) The 1127 glucose-raising rs560887-G allele associates significantly ( $q$-value<0.01) with increased expression of the 1128 long G6PC2 isoform (purple) and reduced expression of the short G6PC2 isoform lacking exon 4 (brown). 


\section{Supplementary Table Legends}

Table S1. Cohort characteristics, genotyping and quality control (QC), glucose, insulin, $2 \mathrm{hGlu}$ and HbA1c analyses and covariates.

Table S2. Association of identified lead coding variants with T2D and anthropometric traits (height, effect allele frequency; Neff: Number of samples in the analysis; BETA: effect size; SE: standard error. Related to Table 1 and Table S3.

Table S3. Coding variant associations in known glycemic trait loci with conditional results on established signals where available. Related to Table 1.

Table S4. Full gene-based results including all variants included in the masks, for both novel and previously-established genes. Related to Table S9.

Table S5. HbA1c-associated loci lookup results for blood cell traits. Related to Table 1.

Table S6. Annotation and classification of effector transcripts into "gold", "silver" and "bronze" categories. Related to Tables 1 and 2 and Figure 1.

Table S7. Gene Set Enrichment Analysis by GeneMANIA network analysis showing enriched GO terms and Reactome pathways in the network for (A) HbA1c; (B) FG; (C) FI; (D) 2hGlu. GOID: Gene Ontology using GO terms and REACTOME gene sets. The enrichment results were considered significant when Bonferroni-adjusted $p$-value $<0.05$ and at least $3 \%$ of the genes contained in the tested gene set is included in the network. Gene sets were also grouped using kappa score into functional groups to improve visualization of enriched pathways. Related to Figures 2 and S1. 
bioRxiv preprint doi: https://doi.org/10.1101/790618; this version posted October 3 2019. The copyright holder for this preprint (which was not certified by peer review) is the author/funder, who has granted bioRxiv a license to display the preprint in perpetuity. It is made available under aCC-BY 4.0 International license.

1159 Table S8. (A-E) EC-DEPICT results (UK Biobank permutations) for (A) all traits combined; (B) all traits 1160 except HbA1c combined; (C) HbA1c only; (D) FG only and (E) 2hGlu only. (F-I) EC-DEPICT results

1161 (Swedish permutations) for (F) all traits combined; (G) all traits except HbA1c combined; (H) HbA1c

1162 only and (I) FG only. Related to Figures 2, S2 and S3.

1163

1164 Table S9. Full G6PC2 gene-based results and conditional analyses for FG and HbA1c. Related to Tables 11652 and S4. 


\section{References}

Abraham, A., Pedregosa, F., Eickenberg, M., Gervais, P., Mueller, A., Kossaifi, J., Gramfort, A., Thirion, B., and Varoquaux, G. (2014). Machine learning for neuroimaging with scikit-learn. Front Neuroinform 8 , 14.

Almgren, P., Lindqvist, A., Krus, U., Hakaste, L., Ottosson-Laakso, E., Asplund, O., Sonestedt, E., Prasad, R.B., Laurila, E., Orho-Melander, M., et al. (2017). Genetic determinants of circulating GIP and GLP-1 concentrations. JCl Insight 2. Astle, W.J., Elding, H., Jiang, T., Allen, D., Ruklisa, D., Mann, A.L., Mead, D., Bouman, H., Riveros-Mckay, F., Kostadima, M.A., et al. (2016). The Allelic Landscape of Human Blood Cell Trait Variation and Links to Common Complex Disease. Cell 167, 1415-1429 e1419.

Baerenwald, D.A., Bonnefond, A., Bouatia-Naji, N., Flemming, B.P., Umunakwe, O.C., Oeser, J.K., Pound, L.D., Conley, N.L., Cauchi, S., Lobbens, S., et al. (2013). Multiple functional polymorphisms in the G6PC2 gene contribute to the association with higher fasting plasma glucose levels. Diabetologia $56,1306-$ 1316.

Barrett, J.C., Clayton, D.G., Concannon, P., Akolkar, B., Cooper, J.D., Erlich, H.A., Julier, C., Morahan, G., Nerup, J., Nierras, C., et al. (2009). Genome-wide association study and meta-analysis find that over 40 loci affect risk of type 1 diabetes. Nat Genet 41, 703-707.

Battle, A., Brown, C.D., Engelhardt, B.E., and Montgomery, S.B. (2017). Genetic effects on gene expression across human tissues. Nature 550, 204-213.

Bindea, G., Mlecnik, B., Hackl, H., Charoentong, P., Tosolini, M., Kirilovsky, A., Fridman, W.H., Pages, F., Trajanoski, Z., and Galon, J. (2009). ClueGO: a Cytoscape plug-in to decipher functionally grouped gene ontology and pathway annotation networks. Bioinformatics 25, 1091-1093.

Bouatia-Naji, N., Bonnefond, A., Baerenwald, D.A., Marchand, M., Bugliani, M., Marchetti, P., Pattou, F., Printz, R.L., Flemming, B.P., Umunakwe, O.C., et al. (2010). Genetic and functional assessment of the role of the rs13431652-A and rs573225-A alleles in the G6PC2 promoter that are strongly associated with elevated fasting glucose levels. Diabetes 59, 2662-2671.

Bouatia-Naji, N., Rocheleau, G., Van Lommel, L., Lemaire, K., Schuit, F., Cavalcanti-Proenca, C., Marchand, M., Hartikainen, A.L., Sovio, U., De Graeve, F., et al. (2008). A polymorphism within the G6PC2 gene is associated with fasting plasma glucose levels. Science 320, 1085-1088.

Carrat, G.R., Hu, M., Nguyen-Tu, M.S., Chabosseau, P., Gaulton, K.J., van de Bunt, M., Siddiq, A., Falchi, M., Thurner, M., Canouil, M., et al. (2017). Decreased STARD10 Expression Is Associated with Defective Insulin Secretion in Humans and Mice. Am J Hum Genet 100, 238-256.

Chen, W.M., Erdos, M.R., Jackson, A.U., Saxena, R., Sanna, S., Silver, K.D., Timpson, N.J., Hansen, T., Orru, M., Grazia Piras, M., et al. (2008). Variations in the G6PC2/ABCB11 genomic region are associated with fasting glucose levels. J Clin Invest 118, 2620-2628.

Chou, J.Y., and Mansfield, B.C. (2008). Mutations in the glucose-6-phosphatase-alpha (G6PC) gene that cause type la glycogen storage disease. Hum Mutat 29, 921-930.

Cohen, R.M., Franco, R.S., Khera, P.K., Smith, E.P., Lindsell, C.J., Ciraolo, P.J., Palascak, M.B., and Joiner, C.H. (2008). Red cell life span heterogeneity in hematologically normal people is sufficient to alter HbA1c. Blood 112, 4284-4291.

Croft, D., Mundo, A.F., Haw, R., Milacic, M., Weiser, J., Wu, G., Caudy, M., Garapati, P., Gillespie, M., Kamdar, M.R., et al. (2014). The Reactome pathway knowledgebase. Nucleic Acids Res 42, D472-477. Dewey, F.E., Murray, M.F., Overton, J.D., Habegger, L., Leader, J.B., Fetterolf, S.N., O'Dushlaine, C., Van 
Esteghamat, F., Broughton, J.S., Smith, E., Cardone, R., Tyagi, T., Guerra, M., Szabo, A., Ugwu, N., Mani, M.V., Azari, B., et al. (2019). CELA2A mutations predispose to early-onset atherosclerosis and metabolic syndrome and affect plasma insulin and platelet activation. Nat Genet 51, 1233-1243. Fehrmann, R.S., Karjalainen, J.M., Krajewska, M., Westra, H.J., Maloney, D., Simeonov, A., Pers, T.H., Hirschhorn, J.N., Jansen, R.C., Schultes, E.A., et al. (2015). Gene expression analysis identifies global gene dosage sensitivity in cancer. Nat Genet 47, 115-125.

1218

1219 Flannick, J., Thorleifsson, G., Beer, N.L., Jacobs, S.B., Grarup, N., Burtt, N.P., Mahajan, A., Fuchsberger, C., Atzmon, G., Benediktsson, R., et al. (2014). Loss-of-function mutations in SLC30A8 protect against type 2 diabetes. Nat Genet 46, 357-363.

1221

1222

1223

1224

1225

1226

1227

1228

1229

1230

1231

1232

1233

1234

1235

Foster, J.D., Pederson, B.A., and Nordlie, R.C. (1997). Glucose-6-phosphatase structure, regulation, and function: an update. Proc Soc Exp Biol Med 215, 314-332.

Franz, M., Rodriguez, H., Lopes, C., Zuberi, K., Montojo, J., Bader, G.D., and Morris, Q. (2018).

GeneMANIA update 2018. Nucleic acids research 46, W60-W64.

Frey, B.J., and Dueck, D. (2007). Clustering by passing messages between data points. Science $315,972-$ 976.

Froguel, P., Vaxillaire, M., Sun, F., Velho, G., Zouali, H., Butel, M.O., Lesage, S., Vionnet, N., Clement, K., Fougerousse, F., et al. (1992). Close linkage of glucokinase locus on chromosome $7 p$ to early-onset noninsulin-dependent diabetes mellitus. Nature 356, 162-164.

Gloyn, A.L., Pearson, E.R., Antcliff, J.F., Proks, P., Bruining, G.J., Slingerland, A.S., Howard, N., Srinivasan, S., Silva, J.M., Molnes, J., et al. (2004). Activating mutations in the gene encoding the ATP-sensitive potassium-channel subunit Kir6.2 and permanent neonatal diabetes. N Engl J Med 350, 1838-1849. Goodarzi, M.O., Cui, J., Chen, Y.D., Hsueh, W.A., Guo, X., and Rotter, J.I. (2011). Fasting insulin reflects heterogeneous physiological processes: role of insulin clearance. Am J Physiol Endocrinol Metab 301, E402-408.

1236

1237

1238

1239

1240

1241

1242

1243

1244

1245

1246 L.A., Fornage, M., et al. (2013). Best practices and joint calling of the HumanExome BeadChip: the CHARGE Consortium. PLoS One 8, e68095.

Gu, Z., Eils, R., and Schlesner, M. (2016). Complex heatmaps reveal patterns and correlations in multidimensional genomic data. Bioinformatics 32, 2847-2849.

Hanson, D., Murray, P.G., Sud, A., Temtamy, S.A., Aglan, M., Superti-Furga, A., Holder, S.E., Urquhart, J., Hilton, E., Manson, F.D., et al. (2009). The primordial growth disorder 3-M syndrome connects ubiquitination to the cytoskeletal adaptor OBSL1. Am J Hum Genet 84, 801-806.

Hara, K., Fujita, H., Johnson, T.A., Yamauchi, T., Yasuda, K., Horikoshi, M., Peng, C., Hu, C., Ma, R.C., Imamura, M., et al. (2014). Genome-wide association study identifies three novel loci for type 2 diabetes. Hum Mol Genet 23, 239-246.

Hart, N.J., Aramandla, R., Poffenberger, G., Fayolle, C., Thames, A.H., Bautista, A., Spigelman, A.F., Babon, J.A.B., DeNicola, M.E., Dadi, P.K., et al. (2018). Cystic fibrosis-related diabetes is caused by islet loss and inflammation. JCl Insight 3.

Jia, B., Madsen, L., Petersen, R.K., Techer, N., Kopperud, R., Ma, T., Doskeland, S.O., Ailhaud, G., Wang, J., Amri, E.Z., et al. (2012). Activation of protein kinase A and exchange protein directly activated by cAMP promotes adipocyte differentiation of human mesenchymal stem cells. PLoS One 7, e34114. Kycia, I., Wolford, B.N., Huyghe, J.R., Fuchsberger, C., Vadlamudi, S., Kursawe, R., Welch, R.P., Albanus, Evolutionarily Conserved Islet Stretch Enhancer and Increases C2CD4A and C2CD4B Expression. Am J Hum Genet 102, 620-635.

Lei, K.J., Chen, Y.T., Chen, H., Wong, L.J., Liu, J.L., McConkie-Rosell, A., Van Hove, J.L., Ou, H.C., Yeh, N.J., Pan, L.Y., et al. (1995). Genetic basis of glycogen storage disease type 1a: prevalent mutations at the

1259 glucose-6-phosphatase locus. Am J Hum Genet 57, 766-771. 
Lesage, S., Drouet, V., Majounie, E., Deramecourt, V., Jacoupy, M., Nicolas, A., Cormier-Dequaire, F., Hassoun, S.M., Pujol, C., Ciura, S., et al. (2016). Loss of VPS13C Function in Autosomal-Recessive Parkinsonism Causes Mitochondrial Dysfunction and Increases PINK1/Parkin-Dependent Mitophagy. Am J Hum Genet 98, 500-513.

1264 Liu, S., Liu, Y., Zhang, Q., Wu, J., Liang, J., Yu, S., Wei, G.H., White, K.P., and Wang, X. (2017). Systematic identification of regulatory variants associated with cancer risk. Genome Biol 18, 194. Liu, X., Jian, X., and Boerwinkle, E. (2013). dbNSFP v2.0: a database of human non-synonymous SNVs and their functional predictions and annotations. Hum Mutat 34, E2393-2402. Mahajan, A., Sim, X., Ng, H.J., Manning, A., Rivas, M.A., Highland, H.M., Locke, A.E., Grarup, N., Im, H.K., Cingolani, P., et al. (2015). Identification and functional characterization of G6PC2 coding variants influencing glycemic traits define an effector transcript at the G6PC2-ABCB11 locus. PLoS Genet 11, e1004876.

1274 Mahajan, A., Taliun, D., Thurner, M., Robertson, N.R., Torres, J.M., Rayner, N.W., Payne, A.J., Steinthorsdottir, V., Scott, R.A., Grarup, N., et al. (2018a). Fine-mapping type 2 diabetes loci to singlevariant resolution using high-density imputation and islet-specific epigenome maps. Nat Genet 50, 15051513. D., Rayner, N.W., et al. (2018b). Refining the accuracy of validated target identification through coding variant fine-mapping in type 2 diabetes. Nat Genet 50, 559-571.

Manning, A.K., Hivert, M.F., Scott, R.A., Grimsby, J.L., Bouatia-Naji, N., Chen, H., Rybin, D., Liu, C.T., Bielak, L.F., Prokopenko, I., et al. (2012). A genome-wide approach accounting for body mass index identifies genetic variants influencing fasting glycemic traits and insulin resistance. Nat Genet 44, 659669.

Marouli, E., Graff, M., Medina-Gomez, C., Lo, K.S., Wood, A.R., Kjaer, T.R., Fine, R.S., Lu, Y., Schurmann, C., Highland, H.M., et al. (2017). Rare and low-frequency coding variants alter human adult height. Nature 542, 186-190.

Mehta, Z.B., Fine, N., Pullen, T.J., Cane, M.C., Hu, M., Chabosseau, P., Meur, G., Velayos-Baeza, A., Monaco, A.P., Marselli, L., et al. (2016). Changes in the expression of the type 2 diabetes-associated gene VPS13C in the beta-cell are associated with glucose intolerance in humans and mice. Am J Physiol Endocrinol Metab 311, E488-507.

1290

1291

1292

1293

1294 Mithieux, G. (1997). New knowledge regarding glucose-6 phosphatase gene and protein and their roles in the regulation of glucose metabolism. Eur J Endocrinol 136, 137-145.

Montague, C.T., Farooqi, I.S., Whitehead, J.P., Soos, M.A., Rau, H., Wareham, N.J., Sewter, C.P., Digby, J.E., Mohammed, S.N., Hurst, J.A., et al. (1997). Congenital leptin deficiency is associated with severe early-onset obesity in humans. Nature 387, 903-908.

Morris, A.P., Voight, B.F., Teslovich, T.M., Ferreira, T., Segre, A.V., Steinthorsdottir, V., Strawbridge, R.J., Khan, H., Grallert, H., Mahajan, A., et al. (2012). Large-scale association analysis provides insights into the genetic architecture and pathophysiology of type 2 diabetes. Nat Genet 44, 981-990.

O'Hare, E.A., Yerges-Armstrong, L.M., Perry, J.A., Shuldiner, A.R., and Zaghloul, N.A. (2016). Assignment of Functional Relevance to Genes at Type 2 Diabetes-Associated Loci Through Investigation of beta-Cell

1300 Mass Deficits. Mol Endocrinol 30, 429-445.

Ongen, H., Buil, A., Brown, A.A., Dermitzakis, E.T., and Delaneau, O. (2016). Fast and efficient QTL

1303

1304

1305

1306 mapper for thousands of molecular phenotypes. Bioinformatics 32, 1479-1485.

Patro, R., Duggal, G., Love, M.I., Irizarry, R.A., and Kingsford, C. (2017). Salmon provides fast and biasaware quantification of transcript expression. Nat Methods 14, 417-419.

Pers, T.H., Karjalainen, J.M., Chan, Y., Westra, H.J., Wood, A.R., Yang, J., Lui, J.C., Vedantam, S., Gustafsson, S., Esko, T., et al. (2015). Biological interpretation of genome-wide association studies using 1307 predicted gene functions. Nat Commun 6, 5890. 
Pirinen, M., Lappalainen, T., Zaitlen, N.A., Dermitzakis, E.T., Donnelly, P., McCarthy, M.I., and Rivas, M.A. (2015). Assessing allele-specific expression across multiple tissues from RNA-seq read data. Bioinformatics 31, 2497-2504. Rosendahl, J., Kirsten, H., Hegyi, E., Kovacs, P., Weiss, F.U., Laumen, H., Lichtner, P., Ruffert, C., Chen, J.M., Masson, E., et al. (2017). Genome-wide association study identifies inversion in the CTRB1-CTRB2 locus to modify risk for alcoholic and non-alcoholic chronic pancreatitis. Gut.

Scarl, R.T., Lawrence, C.M., Gordon, H.M., and Nunemaker, C.S. (2017). STEAP4: its emerging role in metabolism and homeostasis of cellular iron and copper. J Endocrinol 234, R123-R134. Scott, R.A., Lagou, V., Welch, R.P., Wheeler, E., Montasser, M.E., Luan, J., Magi, R., Strawbridge, R.J., Rehnberg, E., Gustafsson, S., et al. (2012). Large-scale association analyses identify new loci influencing glycemic traits and provide insight into the underlying biological pathways. Nat Genet 44, 991-1005. Sever, S., Weinstein, D.A., Wolfsdorf, J.I., Gedik, R., and Schaefer, E.J. (2012). Glycogen storage disease type la: linkage of glucose, glycogen, lactic acid, triglyceride, and uric acid metabolism. J Clin Lipidol 6, 596-600.

Shieh, J.J., Terzioglu, M., Hiraiwa, H., Marsh, J., Pan, C.J., Chen, L.Y., and Chou, J.Y. (2002). The molecular basis of glycogen storage disease type 1a: structure and function analysis of mutations in glucose-6phosphatase. J Biol Chem 277, 5047-5053.

Sikkeland, J., Sheng, X., Jin, Y., and Saatcioglu, F. (2016). STAMPing at the crossroads of normal physiology and disease states. Mol Cell Endocrinol 425, 26-36.

Soranzo, N., Sanna, S., Wheeler, E., Gieger, C., Radke, D., Dupuis, J., Bouatia-Naji, N., Langenberg, C., Prokopenko, I., Stolerman, E., et al. (2010). Common variants at 10 genomic loci influence hemoglobin $A(1)(C)$ levels via glycemic and nonglycemic pathways. Diabetes 59, 3229-3239.

Steinthorsdottir, V., Thorleifsson, G., Sulem, P., Helgason, H., Grarup, N., Sigurdsson, A., Helgadottir, H.T., Johannsdottir, H., Magnusson, O.T., Gudjonsson, S.A., et al. (2014). Identification of low-frequency and rare sequence variants associated with elevated or reduced risk of type 2 diabetes. Nat Genet 46 , 294-298.

Sveinbjornsson, G., Albrechtsen, A., Zink, F., Gudjonsson, S.A., Oddson, A., Masson, G., Holm, H., Kong, A., Thorsteinsdottir, U., Sulem, P., et al. (2016). Weighting sequence variants based on their annotation increases power of whole-genome association studies. Nat Genet 48, 314-317.

t Hart, L.M., Fritsche, A., Nijpels, G., van Leeuwen, N., Donnelly, L.A., Dekker, J.M., Alssema, M., Fadista, J., Carlotti, F., Gjesing, A.P., et al. (2013). The CTRB1/2 locus affects diabetes susceptibility and treatment via the incretin pathway. Diabetes 62, 3275-3281.

Tewhey, R., Kotliar, D., Park, D.S., Liu, B., Winnicki, S., Reilly, S.K., Andersen, K.G., Mikkelsen, T.S., Lander, E.S., Schaffner, S.F., et al. (2016). Direct Identification of Hundreds of Expression-Modulating Variants using a Multiplexed Reporter Assay. Cell 165, 1519-1529.

Thomsen, S.K., Raimondo, A., Hastoy, B., Sengupta, S., Dai, X.Q., Bautista, A., Censin, J., Payne, A.J., Umapathysivam, M.M., Spigelman, A.F., et al. (2018). Type 2 diabetes risk alleles in PAM impact insulin release from human pancreatic beta-cells. Nat Genet 50, 1122-1131.

Turcot, V., Lu, Y., Highland, H.M., Schurmann, C., Justice, A.E., Fine, R.S., Bradfield, J.P., Esko, T., Giri, A., Graff, M., et al. (2018). Protein-altering variants associated with body mass index implicate pathways that control energy intake and expenditure in obesity. Nat Genet 50, 26-41.

Ulirsch, J.C., Nandakumar, S.K., Wang, L., Giani, F.C., Zhang, X., Rogov, P., Melnikov, A., McDonel, P., Do, R., Mikkelsen, T.S., et al. (2016). Systematic Functional Dissection of Common Genetic Variation Affecting Red Blood Cell Traits. Cell 165, 1530-1545. van de Bunt, M., Manning Fox, J.E., Dai, X., Barrett, A., Grey, C., Li, L., Bennett, A.J., Johnson, P.R., Rajotte, R.V., Gaulton, K.J., et al. (2015). Transcript Expression Data from Human Islets Links Regulatory Signals from Genome-Wide Association Studies for Type 2 Diabetes and Glycemic Traits to Their Downstream Effectors. PLoS Genet 11, e1005694. 
Wessel, J., Chu, A.Y., Willems, S.M., Wang, S., Yaghootkar, H., Brody, J.A., Dauriz, M., Hivert, M.F., Raghavan, S., Lipovich, L., et al. (2015). Low-frequency and rare exome chip variants associate with fasting glucose and type 2 diabetes susceptibility. Nat Commun 6, 5897. Wheeler, E., Leong, A., Liu, C.T., Hivert, M.F., Strawbridge, R.J., Podmore, C., Li, M., Yao, J., Sim, X., Hong, J., et al. (2017a). Impact of common genetic determinants of Hemoglobin A1c on type 2 diabetes risk and diagnosis in ancestrally diverse populations: A transethnic genome-wide meta-analysis. PLoS Med 14, e1002383. insights. Hum Mol Genet 26, R172-R184. Williams, A.L., Jacobs, S.B., Moreno-Macias, H., Huerta-Chagoya, A., Churchhouse, C., Marquez-Luna, C., Garcia-Ortiz, H., Gomez-Vazquez, M.J., Burtt, N.P., Aguilar-Salinas, C.A., et al. (2014). Sequence variants in SLC16A11 are a common risk factor for type 2 diabetes in Mexico. Nature 506, 97-101. Wolpin, B.M., Rizzato, C., Kraft, P., Kooperberg, C., Petersen, G.M., Wang, Z., Arslan, A.A., BeaneFreeman, L., Bracci, P.M., Buring, J., et al. (2014). Genome-wide association study identifies multiple susceptibility loci for pancreatic cancer. Nat Genet 46, 994-1000. Woodmansey, C., McGovern, A.P., McCullough, K.A., Whyte, M.B., Munro, N.M., Correa, A.C., Gatenby, P.A.C., Jones, S.A., and de Lusignan, S. (2017). Incidence, Demographics, and Clinical Characteristics of Diabetes of the Exocrine Pancreas (Type 3c): A Retrospective Cohort Study. Diabetes Care 40, 14861493.

1375 summary statistics identifies additional variants influencing complex traits. Nat Genet 44, 369-375, S361363. sequencing variants by leveraging the Ensembl Variant Effect Predictor with plugins. Brief Bioinform 16, 255-264. 


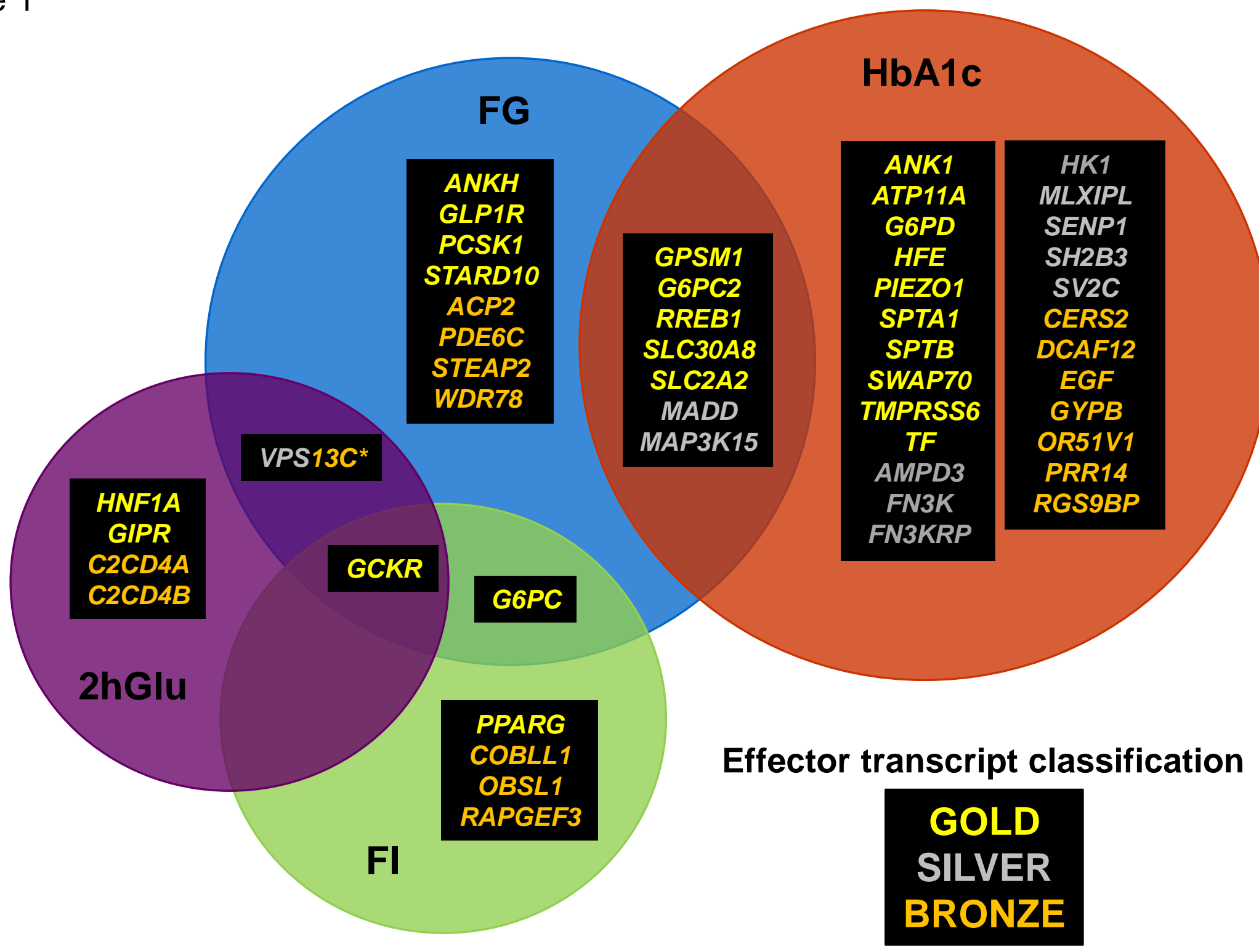

Figure 1. Effector transcript classification into "gold", "silver" and "bronze" categories based on strength of genetic and biological evidence. A total of 51 effector transcripts from 74 single variant and six gene-based signals were identified, with many of them shared across traits. The classification was undertaken independently by four of the authors and the consensus was used as the final classification for effector transcripts (see Methods). *Asterisk indicates "silver" for FG, "bronze" for 2hGlu. 


\section{Figure 2}

A HbA1c network

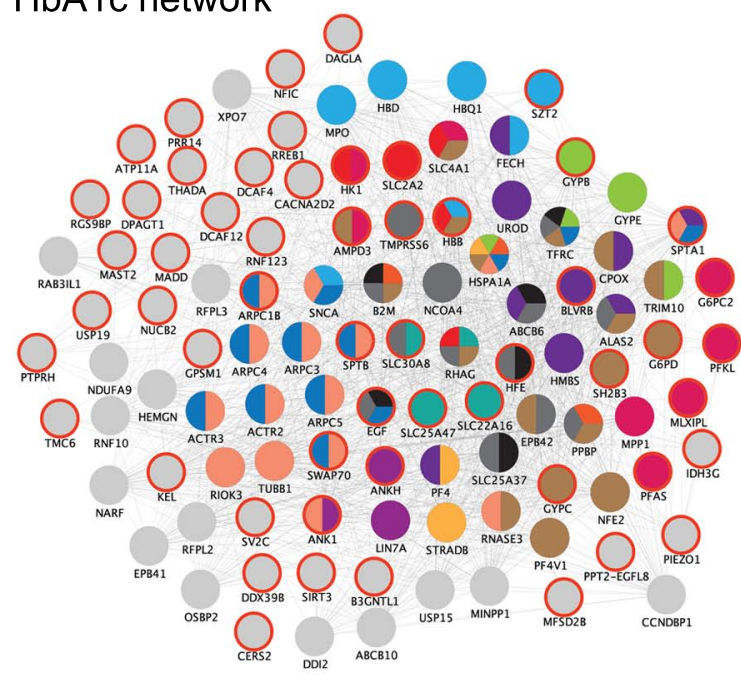

C

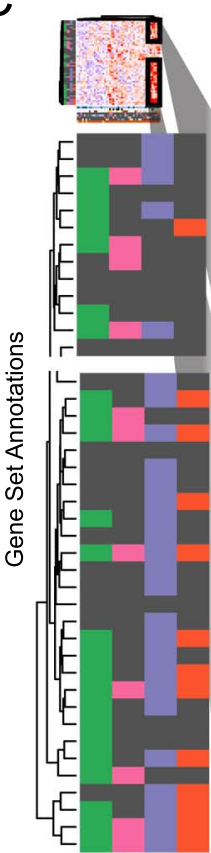

\section{All Traits Except $\mathrm{HbA1c}$}

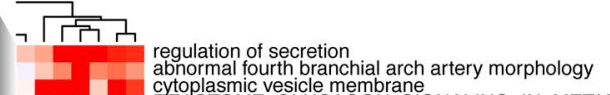

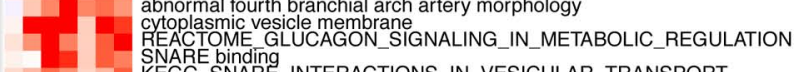

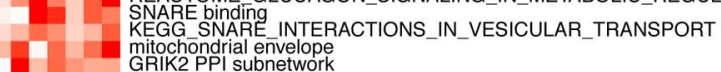

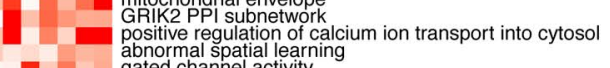

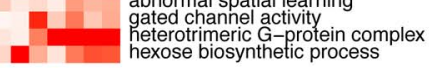

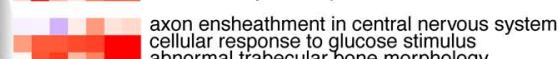

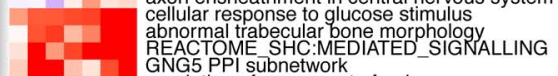

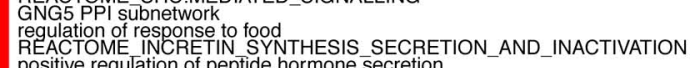

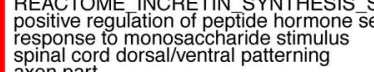

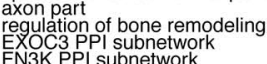

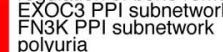

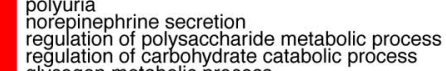

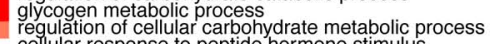

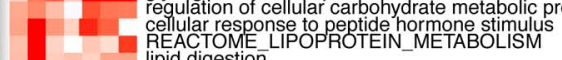

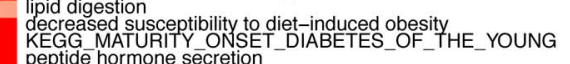

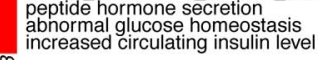

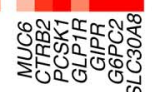
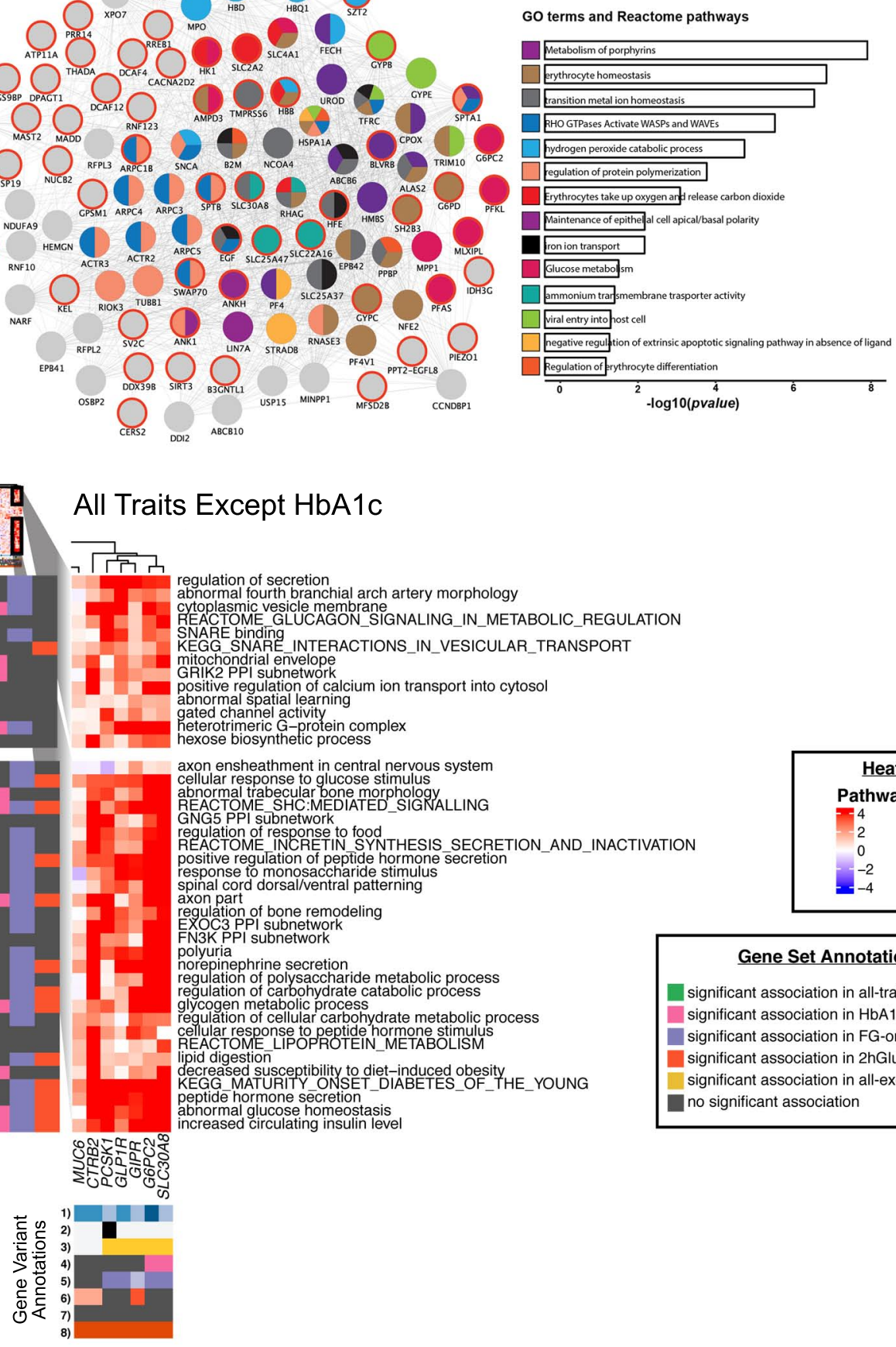

B FG network

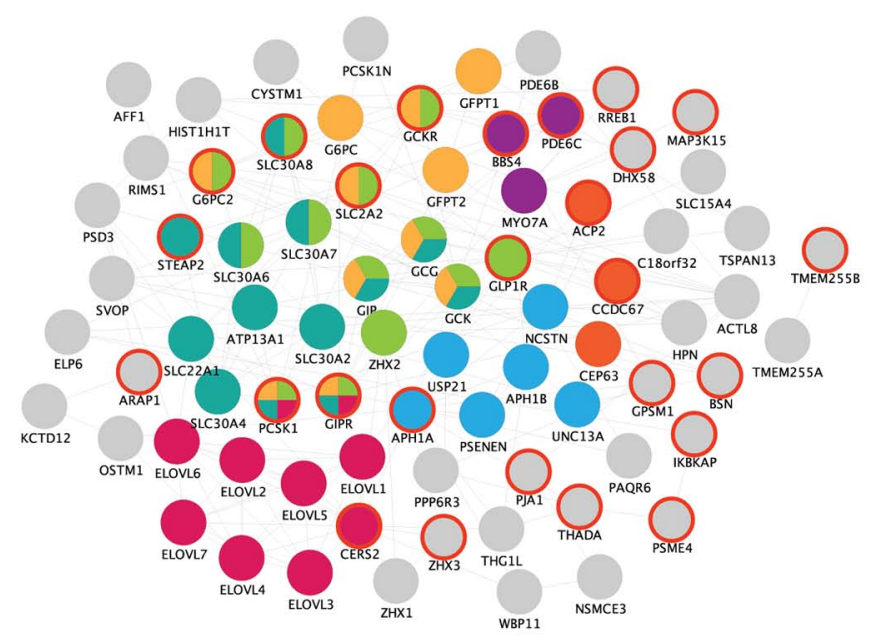

GO terms and Reactome pathways

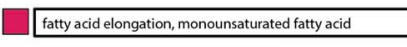

Zinc efflux and compartmentalization

$\square$ Noncanonical activation of NoTCH3

Insulinsecertion

$\square$ Glucose metabolil m

Eye photorefeptoto cell development

$\square$ Centrioleterdolication

${ }_{-\log 10(\text { pvalue })}^{7.5}$

D

\section{Fasting Glucose}

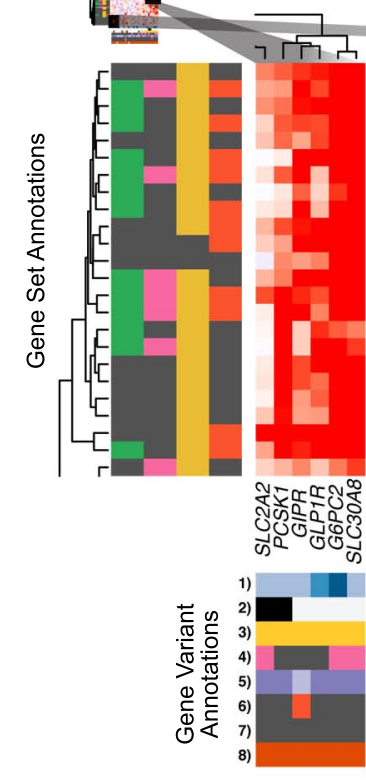

Heatmap Fill

Path
-4
-2
0

0
-2
-4

Gene Set Annotations

significant association in all-trait analysis significant association in HbA1c-only analysis significant association in FG-only analysis
significant association in 2hGlu-only analysis significant association in all-except-HbA1c analys no significant association

\begin{tabular}{|c|c|c|c|c|c|c|c|}
\hline \multirow[b]{2}{*}{$\begin{array}{l}\text { 1) MAF } \\
\text { rare- } \\
\text { low-frequency } \\
\text { common }\end{array}$} & & Gene Variant A & nnotations & & & & \\
\hline & $\begin{array}{l}\text { 2) OMIM } \\
\text { y } \begin{array}{l}\text { diabetes/glycemic } \\
\text { none }\end{array}\end{array}$ & $\begin{array}{l}\text { 3) Classification } \\
\text { gold } \\
\text { silver } \\
\text { bronze }\end{array}$ & $\begin{array}{l}\text { 4) HbA1c } \\
\text { AWS } \\
\text { n.s. }\end{array}$ & 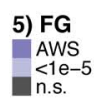 & $\begin{array}{l}\text { 6) 2hGlu } \\
\text { AWS } \\
\text { <1e-5 } \\
\text { n.s. }\end{array}$ & $\begin{array}{l}\text { 7) } \mathrm{FI} \\
\text { AWS } \\
<1<-5 \\
\text { n.s. }\end{array}$ & $\begin{array}{l}\text { 8) Inclusion } \\
\text { included } \\
\text { excluded }\end{array}$ \\
\hline
\end{tabular}

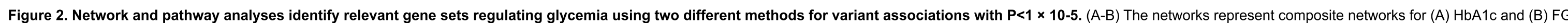

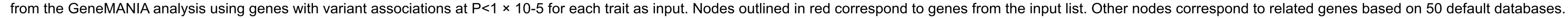

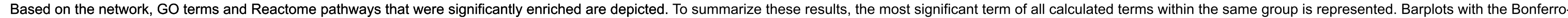
ni-adjusted -log10(p-values) of the most significant terms within each group are are shown. Each group was assigned a specific color; if a gene is present in more than one term, it is displayed in more than one color.

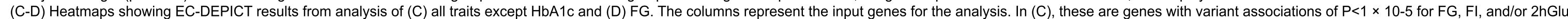

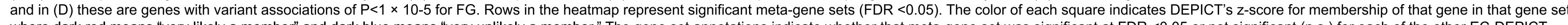

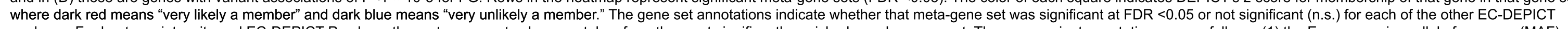

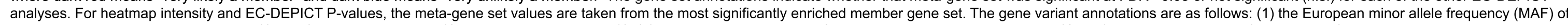

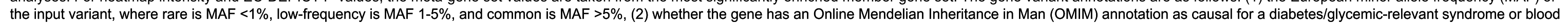

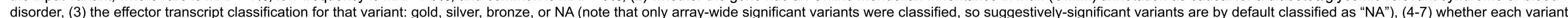

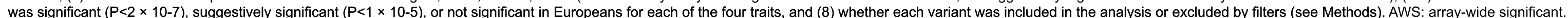
Related to Figures $\mathrm{S} 1$ to $\mathrm{S} 3$. 

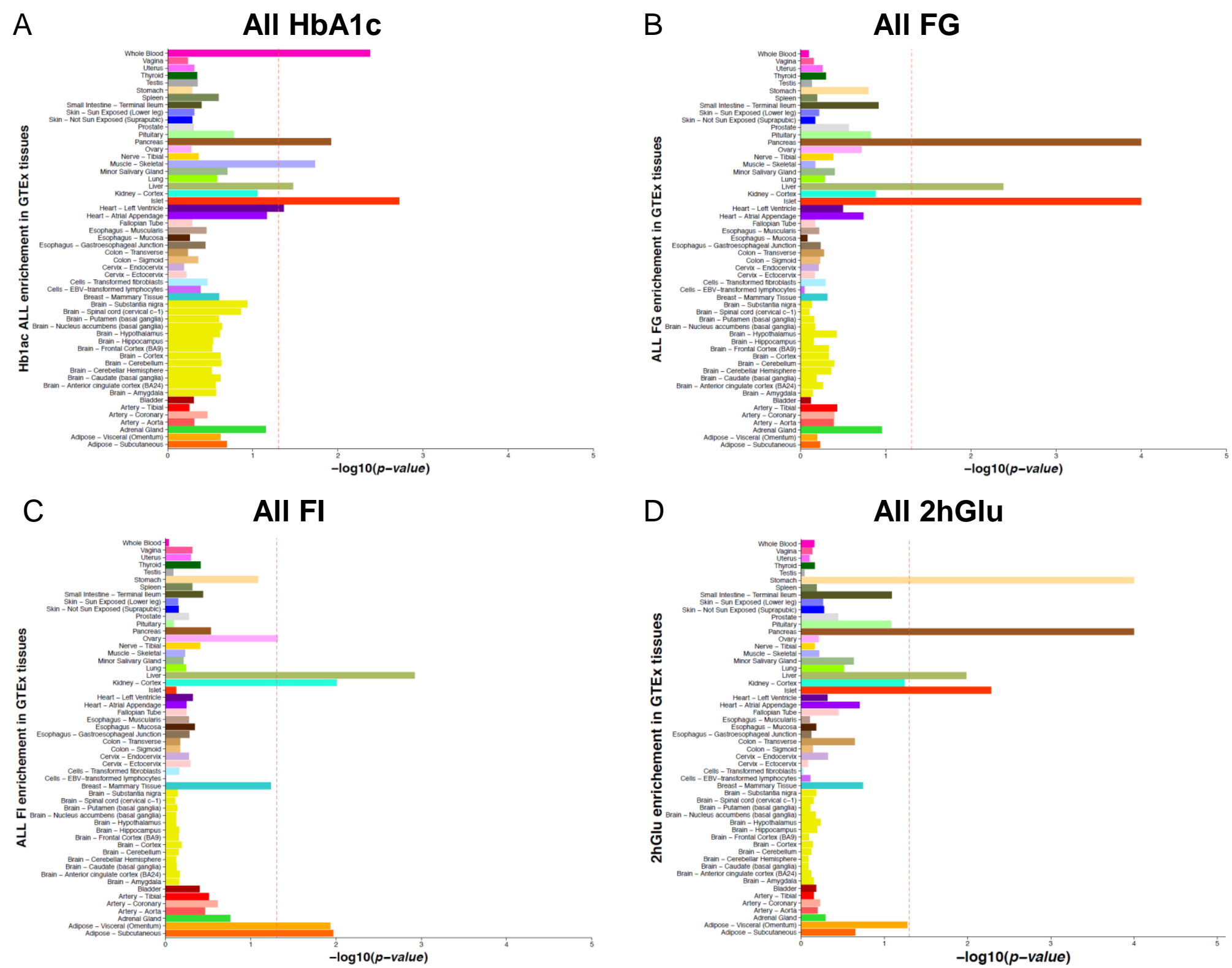

Figure 3. Tissue enrichment analysis reveals the key tissues involved in the regulation of glycemic traits. The figures display expression enrichment of genes from all of the golden, silver, and bronze gene set lists for (A) HbA1c, (B) FG, (C) FI and (D) 2hGlu in GTEx tissue samples plus islet data. Enrichment $P$-values were assessed empirically for each tissue using a permutation procedure (10,000 iterations), and the red vertical line shows the significance threshold (empirical $P<0.05$ ). 
Figure 4 aCC-BY 4.0 International license.
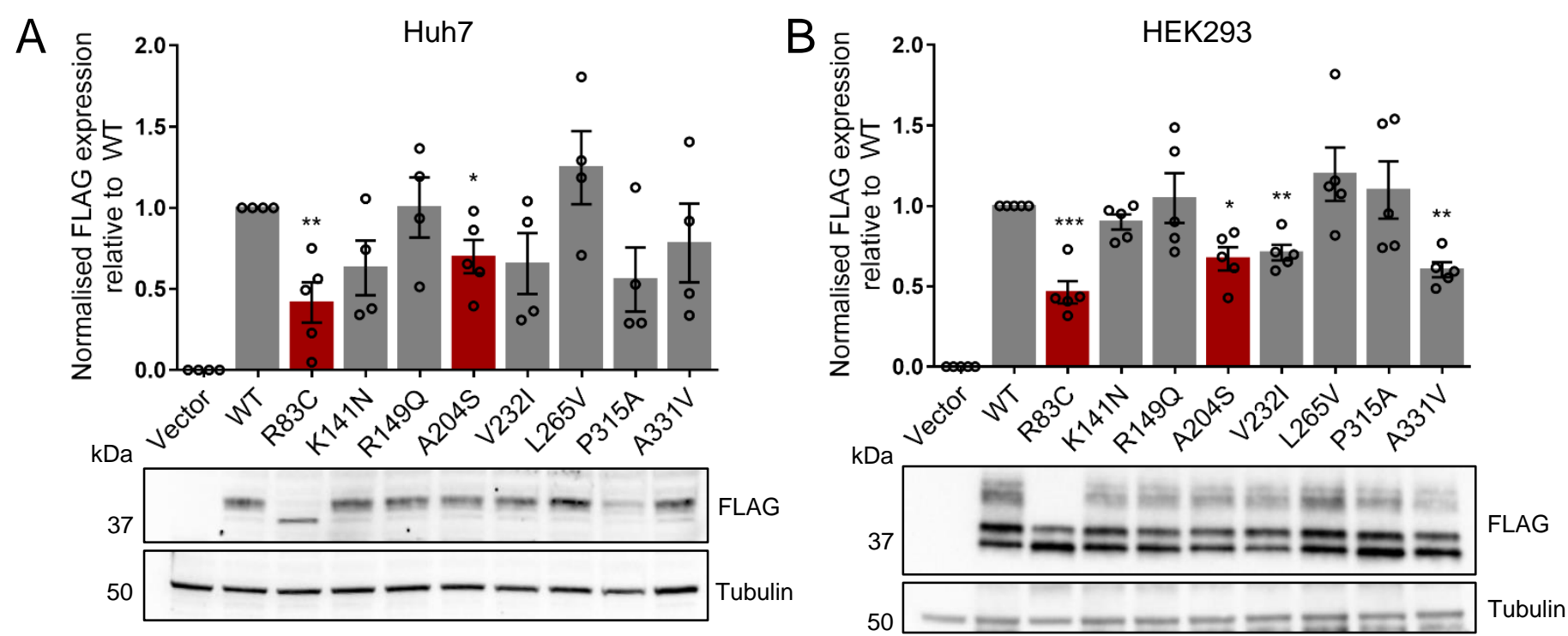

C

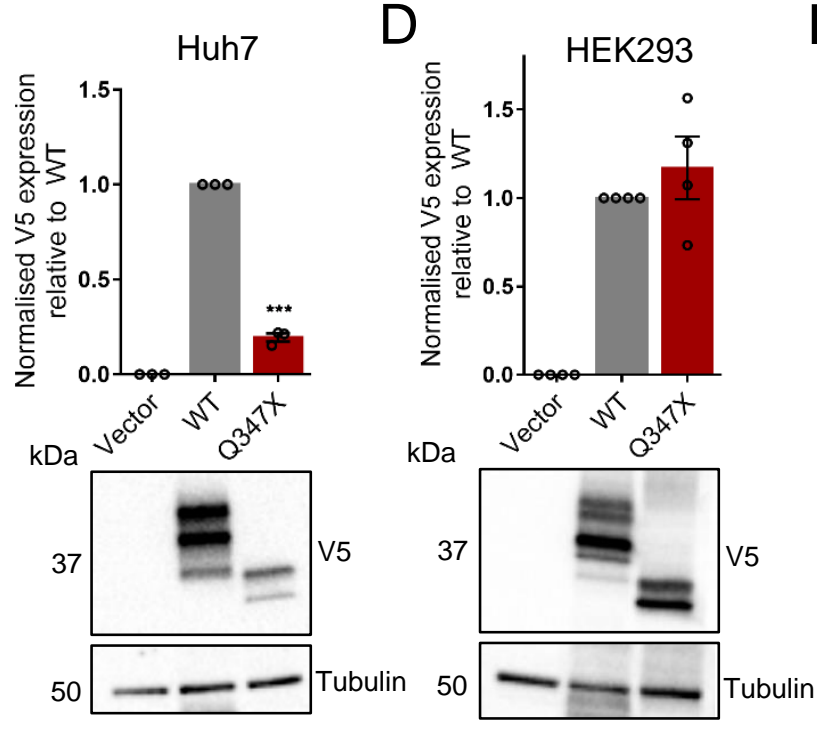

E

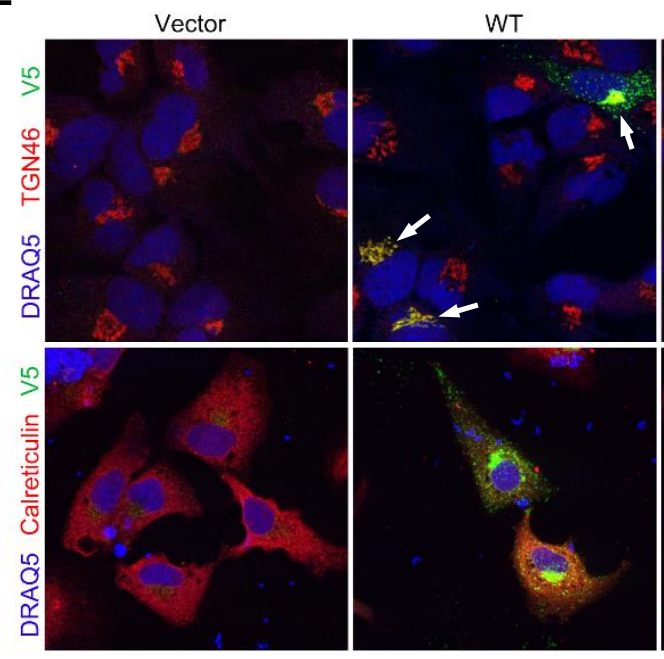

Q347X

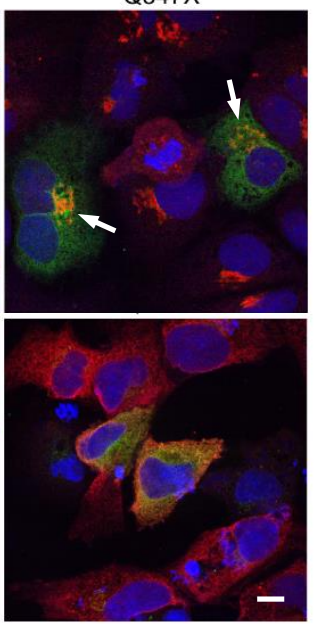

F
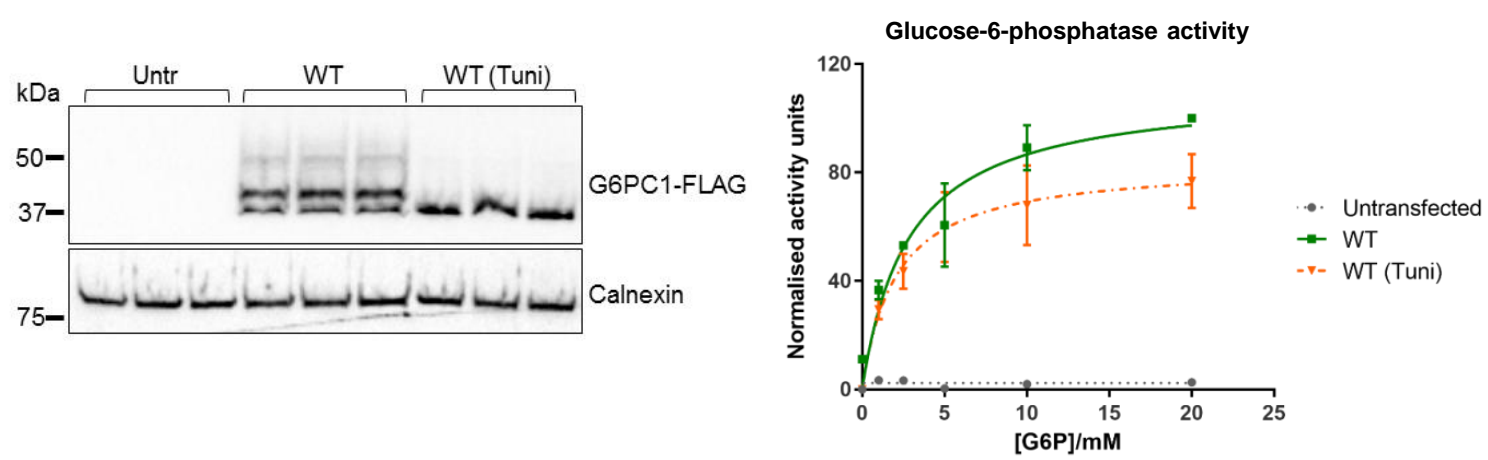
Figure 4. Functional characterisation of G6PC variant proteins. Related to Figure S4.

(A) Protein expression levels of missense G6PC variants were determined in Huh7 cells $(n=4-5)$ and (B) HEK293 cells $(n=5)$ by western blot densitometric analysis of FLAG-tagged G6PC constructs relative to tubulin control, with representative blots shown.

(C) Protein expression levels of PTV Q347X were determined in Huh7 cells $(n=3)$ and (D) HEK293 cells $(n=4)$ by western blot densitometric analysis of V5-tagged G6PC constructs relative to tubulin control, with representative blots shown. Bars in red indicate variants that are statistical drivers of the gene-based signal.

(E) Cellular localisation of V5-tagged G6PC-Q347X was assessed in Huh7 cells and overlaid with markers for the ER (calreticulin) and the trans-golgi network (TGN46). White arrows point to positions of the Golgi apparatus. Scale bar indicates $10 \mu \mathrm{m}$.

(F) Glucose-6-phosphatase activity of unglycosylated WT G6PC protein obtained from tunicamycin-treated (Tuni) HEK293 microsomes $(n=2)$, with representative western blot of microsomal protein shown. All data presented as mean \pm SEM. ${ }^{*} p=0.01-0.05 ;{ }^{* \star} p=0.001$ $0.01 ;{ }^{* *} p<0.001$. 
Figure 5

A

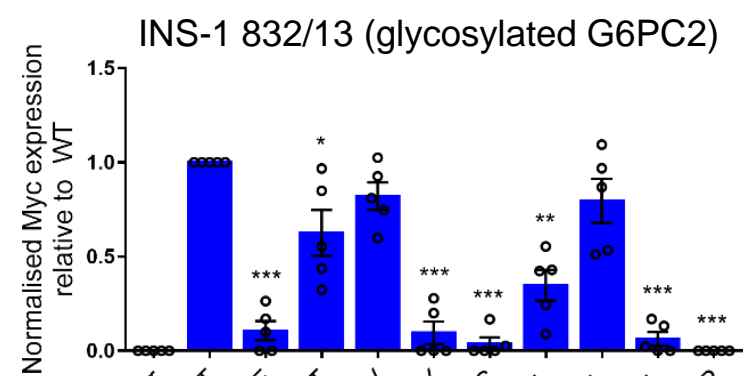

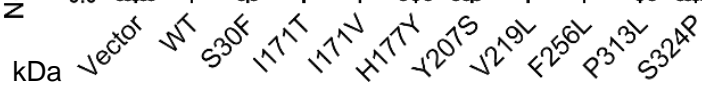

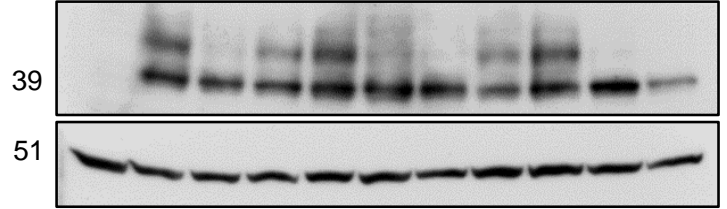

+ MG-132 (10 $\mu \mathrm{M})$

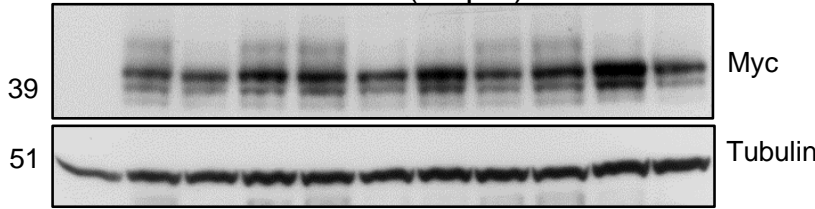

+ Chloroquine $(100 \mu \mathrm{M})$

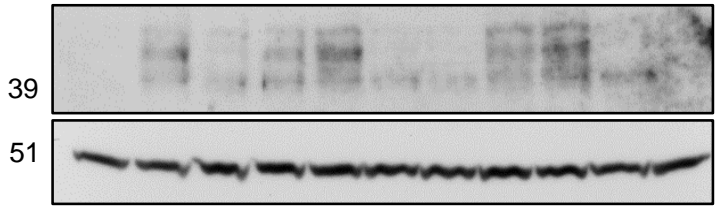

Glucose-6-phosphatase activity

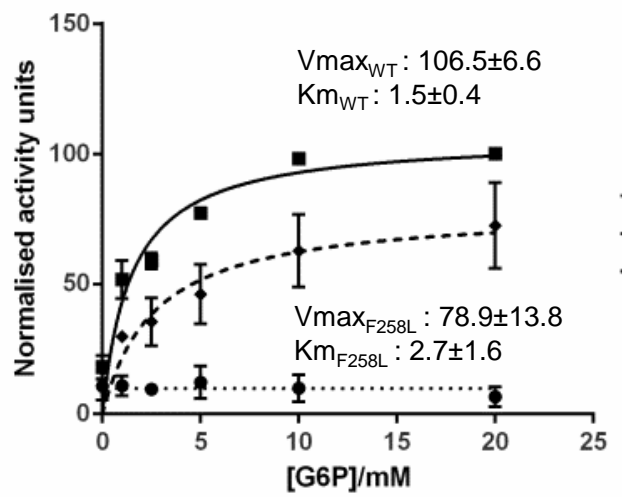

$E$
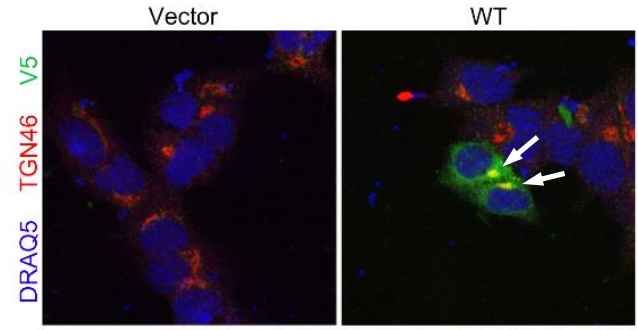

- Untransfected

$\rightarrow$ WT

-↔. F258L
Myc

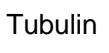
Myc Tubulin
D
B

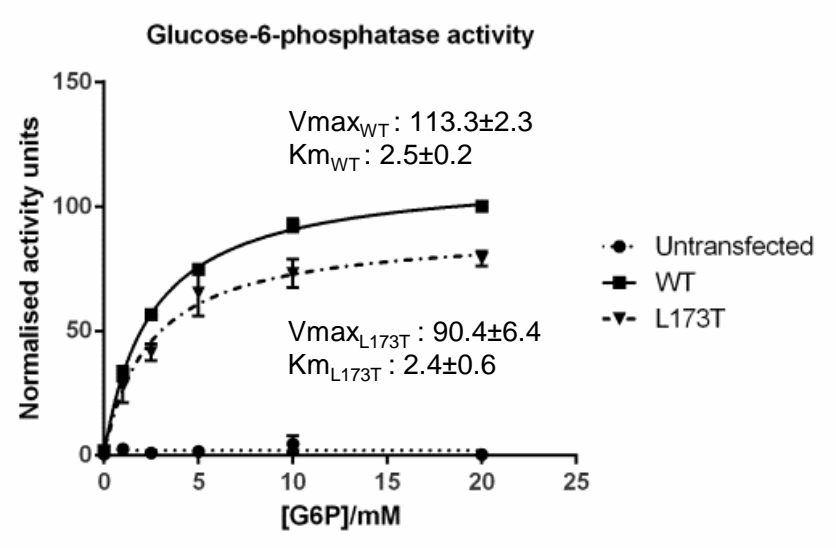

Glucose-6-phosphatase activity

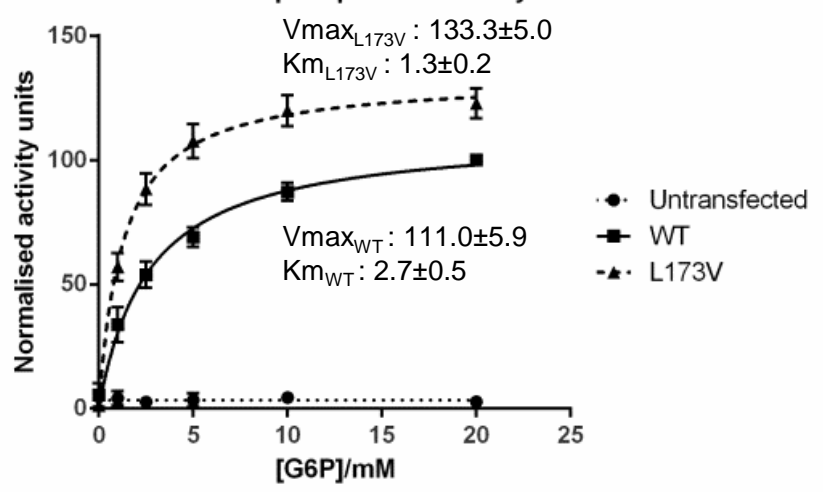

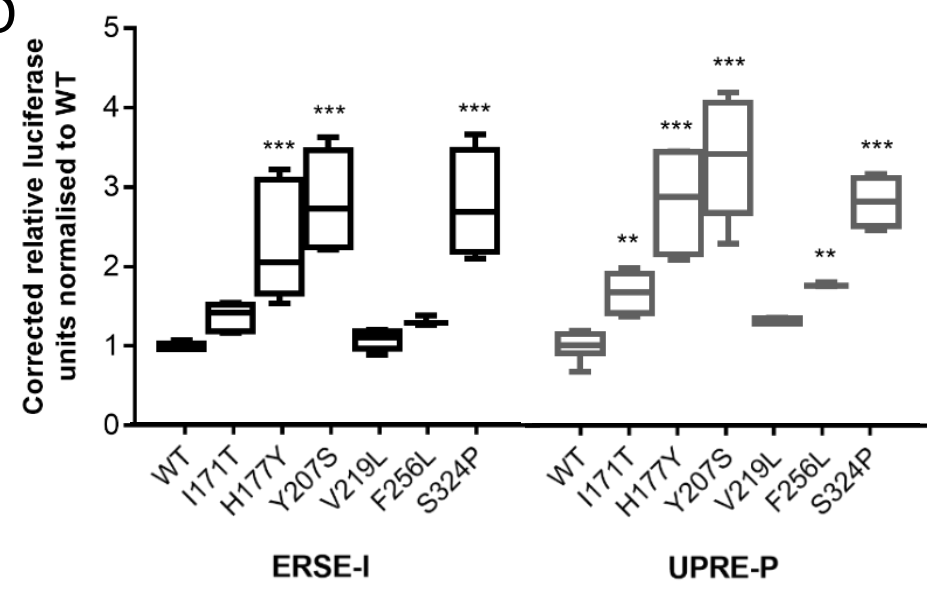

ER stress response element reporter

F

Content-normalised insulin secretion

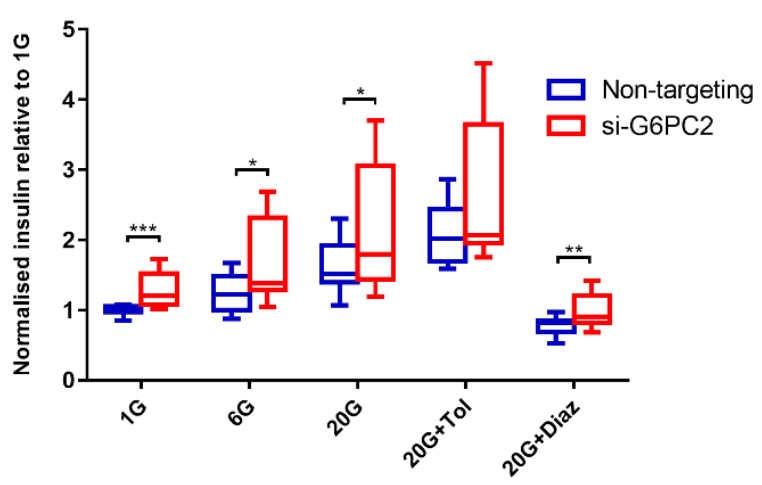

Conditions 
Figure 5. Functional characterisation of G6PC2 variant proteins and the role of G6PC2 in human beta cells. Related to Figure S5.

(A) Expression levels of the glycosylated forms (upper bands only) of G6PC2 variant proteins were determined in INS-1 832/13 cells by western blot densitometric analysis of Myc-tagged G6PC2 constructs relative to tubulin control $(n=5)$. Representative blots are shown for untreated cells together with cells treated with proteasomal inhibitor MG-132 or lysosomal inhibitor chloroquine.

(B) Glucose-6-phosphatase activity of L173T and L173V variants in G6PC (proxy for I171T and I171V in G6PC2 respectively) in HEK293 against increasing glucose-6-phosphate concentrations $(n=4)$, with mean $V \max \pm$ SEM and $\mathrm{Km} \pm$ SEM values shown for WT and each variant.

(C) Glucose-6-phosphatase activity of F258L variant in G6PC (proxy for F256L in G6PC2) in HEK293 against increasing glucose-6-phosphate concentrations $(n=3)$, with mean Vmax \pm $\mathrm{SEM}$ and $\mathrm{Km} \pm \mathrm{SEM}$ values shown. Vmax and $\mathrm{Km}$ results were computed based on the Michaelis-Menten kinetic model.

(D) Effect of G6PC2 WT and variant protein expression on luciferase activity driven by ER stress response elements in HEK293 cells. Relative luciferase units corrected for background activity were normalised to WT for each reporter, from $n=6$ across two independent experiments (except for F256L, $\mathrm{n}=3$ in one experiment) using two-way ANOVA with Fisher's LSD test comparing each variant to WT.

(E) Cellular localisation of R283X in EndoC- $\beta \mathrm{H} 1$ overlaid with markers for the ER (calreticulin) and the trans-golgi network (TGN46). White arrows point to positions of the Golgi apparatus. Scale bar indicates $10 \mu \mathrm{m}$.

(F) Insulin secretion normalised to total content at basal and high glucose conditions (with and without drug treatments) following 96-120h G6PC2 knockdown in EndoC- $\beta \mathrm{H} 1$. Unpaired twotailed Students' t tests were used to compare G6PC2 knockdown to control for each condition, from $\mathrm{n}=16$ across 4 independent experiments. Tol: tolbutamide; Diaz: diazoxide. All data presented as mean \pm SEM. ${ }^{*} p=0.01-0.05 ;{ }^{* *} p=0.001-0.01 ;{ }^{* * *} p<0.001$. 


\begin{tabular}{|c|c|c|c|c|c|c|c|c|c|c|}
\hline $\begin{array}{l}\text { bioRxiy } \\
\text { Tcaidtified }\end{array}$ & $\begin{array}{l}\text { preprint doi: http } \\
\text { d by peef Preview) }\end{array}$ & $\begin{array}{l}\text { ps://doi.org/10 } \\
\text { ) is thenequthor }\end{array}$ & 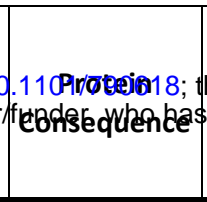 & $\begin{array}{l}\text { hiAlledesso } \\
\text { grented } \\
\text { aCC-BY }\end{array}$ & $\begin{array}{l}\text { Freq. } \\
\text { posted Oc } \\
\text { pioftect lic } \\
\text { 4.0Alltelenati }\end{array}$ & 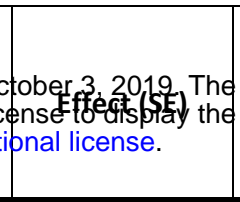 & $\begin{array}{l}\text { copyright hol } \\
\text { preprint in pe }\end{array}$ & $\begin{array}{l}\text { Ider for this } \\
\text { erpetulty. It }\end{array}$ & \begin{tabular}{|c|} 
Previous \\
glycemic \\
spreprint (whiф) \\
t is mataitavail \\
association \\
(if any)
\end{tabular} & $\begin{array}{l}\text { Locus name } \\
\text { hh was not } \\
\text { abferfredtepus } \\
\text { association }\end{array}$ \\
\hline FG & rs1886686 & WDR78 & p.G12A & G/C & 0.739 & $0.014(0.002)$ & $2.24 \times 10^{-11}$ & 123558 & Novel & \\
\hline HbA1c & rs267738 & CERS2 & p.E106A & $\mathrm{G} / \mathrm{T}$ & 0.186 & $-0.01(0.002)$ & $6.96 \times 10^{-10}$ & 144043 & HbA1c & CERS2 \\
\hline HbA1c & rs863362 & OR10X1 & p.W66X & $\mathrm{T} / \mathrm{C}$ & 0.465 & $0.011(0.001)$ & $6.76 \times 10^{-15}$ & 114945 & HbA1c & SPTA1 \\
\hline HbA1c & rs857725 & SPTA1 & p.K1693Q & $\mathrm{G} / \mathrm{T}$ & 0.262 & $0.022(0.001)$ & $1.56 \times 10^{-50}$ & 143956 & $\mathrm{HbA1c}$ & SPTA1 \\
\hline HbA1c & rs11887523 & MFSD2B & p.A60T & $A / G$ & 0.007 & $-0.072(0.01)$ & $1.44 \times 10^{-12}$ & 122060 & HbA1c & ATAD2B \\
\hline FG & rs $1260326^{2}$ & $G C K R$ & p.L446P & $\mathrm{C} / \mathrm{T}$ & 0.631 & $0.029(0.002)$ & $6.36 \times 10^{-48}$ & 129588 & FG, FI, 2hGlu & $G C K R$ \\
\hline $\mathrm{FI}$ & rs $1260326^{2}$ & $G C K R$ & p.L446P & $\mathrm{C} / \mathrm{T}$ & 0.626 & $0.024(0.002)$ & $5.55 \times 10^{-32}$ & 104076 & FG, FI, 2hGlu & $G C K R$ \\
\hline $2 \mathrm{hGlu}$ & rs1260326 ${ }^{2}$ & $G C K R$ & p.L446P & $\mathrm{C} / \mathrm{T}$ & 0.618 & $-0.069(0.009)$ & $4.48 \times 10^{-15}$ & 57813 & FG, FI, 2hGlu & $G C K R$ \\
\hline FG & rs35720761 ${ }^{2}$ & THADA & p.C845Y & $\mathrm{T} / \mathrm{C}$ & 0.108 & $-0.018(0.003)$ & $4.35 \times 10^{-9}$ & 129622 & $\mathrm{~T} 2 \mathrm{D}$ & THADA \\
\hline $\mathrm{HbA1c}$ & rs35720761 ${ }^{2}$ & THADA & p.C845Y & $\mathrm{C} / \mathrm{T}$ & 0.113 & $0.014(0.002)$ & $2.58 \times 10^{-12}$ & 144001 & T2D & THADA \\
\hline FG & rs7578597 & THADA & p.T897A & $\mathrm{C} / \mathrm{T}$ & 0.106 & $-0.019(0.003)$ & $1.99 \times 10^{-8}$ & 113162 & $\mathrm{~T} 2 \mathrm{D}$ & THADA \\
\hline $\mathrm{FI}$ & rs7607980 2 & COBLL1 & p.N901D & $\mathrm{C} / \mathrm{T}$ & 0.128 & $-0.032(0.003)$ & $1.30 \times 10^{-24}$ & 97817 & $\mathrm{FI}$ & COBLL1 \\
\hline FG & rs2232323 & G6PC2 & p.Y207S & $C / A$ & 0.006 & $-0.129(0.012)$ & $1.05 \times 10^{-28}$ & 123981 & $\mathrm{FG}, \mathrm{HbA} 1 \mathrm{c}$ & G6PC2 \\
\hline HbA1c & rs2232323 & G6PC2 & p.Y207S & $\mathrm{C} / \mathrm{A}$ & 0.007 & $-0.053(0.007)$ & $3.25 \times 10^{-13}$ & 144038 & FG, $\mathrm{HbA} 1 \mathrm{c}$ & G6PC2 \\
\hline FG & rs146779637 & G6PC2 & p.R283X & $\mathrm{T} / \mathrm{C}$ & 0.002 & $-0.138(0.02)$ & $1.78 \times 10^{-12}$ & 127278 & FG, $\mathrm{HbA} 1 \mathrm{c}$ & G6PC2 \\
\hline HbA1c & rs146779637 & G6PC2 & p.R283X & $\mathrm{T} / \mathrm{C}$ & 0.002 & $-0.074(0.012)$ & $4.58 \times 10^{-10}$ & 141728 & FG, $\mathrm{HbA} 1 \mathrm{c}$ & G6PC2 \\
\hline FI & rs1983210 & OBSL1 & p.E1365D & G/C & 0.729 & $0.016(0.003)$ & $8.48 \times 10^{-10}$ & 79767 & Novel & \\
\hline FI & rs3183099 & OBSL1 & $\begin{array}{l}\text { splice region } \\
\text { variant }\end{array}$ & A/G & 0.226 & $-0.013(0.002)$ & $4.70 \times 10^{-8}$ & 100713 & Novel & \\
\hline $\mathrm{FI}$ & rs1801282 ${ }^{2}$ & PPARG & p.P12A & $\mathrm{G} / \mathrm{C}$ & 0.117 & $-0.031(0.003)$ & $3.50 \times 10^{-23}$ & 98631 & $\mathrm{FI}$ & PPARG \\
\hline HbA1c & rs35726701 & RNF123 & p.K596E & $\mathrm{G} / \mathrm{A}$ & 0.019 & $0.025(0.005)$ & $4.19 \times 10^{-8}$ & 131203 & HbA1c & USP4 \\
\hline FG & rs5400 & $S L C 2 A 2$ & p.T110I & $A / G$ & 0.161 & $-0.022(0.003)$ & $2.14 \times 10^{-17}$ & 129591 & FG, HbA1c & $S L C 2 A 2$ \\
\hline HbA1c & rs5400 & $S L C 2 A 2$ & p.T110I & $A / G$ & 0.153 & $-0.013(0.002)$ & $2.27 \times 10^{-13}$ & 144012 & FG, HbA1c & $S L C 2 A 2$ \\
\hline $\mathrm{HbA} 1 \mathrm{c}^{1}$ & rs2237051 & $E G F$ & p.M708I & $A / G$ & 0.374 & $-0.007(0.001)$ & $2.11 \times 10^{-7}$ & 121204 & Novel & \\
\hline HbA1c & rs7683365 & GYPB & p.T48M & $A / G$ & 0.312 & $0.012(0.002)$ & $1.61 \times 10^{-8}$ & 45191 & HbA1c & FREM3 \\
\hline FG & rs146886108 ${ }^{2}$ & ANKH & p.R187Q & $\mathrm{T} / \mathrm{C}$ & 0.004 & $-0.088(0.014)$ & $5.67 \times 10^{-10}$ & 129647 & Novel & \\
\hline HbA1c & rs31244 & SV2C & p.D543N & $A / G$ & 0.083 & $0.012(0.002)$ & $6.05 \times 10^{-8}$ & 144000 & Novel & \\
\hline FG & rs6235 & PCSK1 & p.S690T & $\mathrm{G} / \mathrm{C}$ & 0.264 & $-0.022(0.002)$ & $9.22 \times 10^{-24}$ & 123560 & FG & PCSK1 \\
\hline $2 \mathrm{hGlu}$ & rs2549782 & ERAP2 & p.K392N & $T / G$ & 0.519 & $-0.055(0.009)$ & $6.81 \times 10^{-10}$ & 57836 & $2 \mathrm{hGlu}$ & ERAP2 \\
\hline HbA1c & rs35742417 & RREB1 & p.S1499Y & $A / C$ & 0.173 & $-0.01(0.002)$ & $3.76 \times 10^{-9}$ & 143967 & FG, T2D & RREB1 \\
\hline FG & rs35742417 & RREB1 & p.S1499Y & $A / C$ & 0.183 & $-0.019(0.002)$ & $1.27 \times 10^{-16}$ & 129577 & FG, T2D & RREB1 \\
\hline HbA1c & rs1799945 & $H F E$ & p.H63D & $\mathrm{G} / \mathrm{C}$ & 0.129 & $-0.023(0.002)$ & $1.20 \times 10^{-30}$ & 128354 & $\mathrm{HbA1c}$ & $\begin{array}{c}\text { HFE, } \\
\text { HIST1H4A }\end{array}$ \\
\hline HbA1c & rs1800562 & HFE & p.C279Y & $A / G$ & 0.051 & $-0.042(0.003)$ & $3.30 \times 10^{-47}$ & 138093 & $\mathrm{HbA1c}$ & $\begin{array}{c}\text { HFE, } \\
\text { HIST1H4A }\end{array}$ \\
\hline $\mathrm{FG}$ & rs10305492 & GLP1R & p.A316T & $\mathrm{A} / \mathrm{G}$ & 0.014 & $-0.08(0.008)$ & $2.37 \times 10^{-25}$ & 129601 & FG & GLP1R \\
\hline HbA1c & rs35332062 & MLXIPL & p.A358V & A/G & 0.117 & $0.011(0.002)$ & $6.18 \times 10^{-9}$ & 144042 & Novel & \\
\hline HbA1c & rs3812316 & MLXIPL & p.Q241H & G/C & 0.112 & $0.012(0.002)$ & $2.15 \times 10^{-8}$ & 108605 & Novel & \\
\hline FG & rs194524 ${ }^{3}$ & STEAP2 & p.R456Q & $A / G$ & 0.523 & $0.01(0.002)$ & $7.65 \times 10^{-8}$ & 129629 & Novel & \\
\hline HbA1c & rs34664882 & ANK1 & p.A1503V & $A / G$ & 0.026 & $-0.049(0.004)$ & $2.43 \times 10^{-39}$ & 144034 & HbA1c & ANK1 \\
\hline FG & rs $13266634^{2}$ & SLC30A8 & p.R276W & $\mathrm{T} / \mathrm{C}$ & 0.305 & $-0.029(0.002)$ & $1.63 \times 10^{-46}$ & 129614 & $\begin{array}{c}\text { FG, } \mathrm{HbA1c}, \\
\text { T2D }\end{array}$ & SLC30A8 \\
\hline HbA1c & rs13266634 & SLC3OA8 & p.R276W & $\mathrm{T} / \mathrm{C}$ & 0.300 & $-0.015(0.001)$ & $8.50 \times 10^{-28}$ & 143982 & $\begin{array}{c}\text { FG, HbA1c, } \\
\text { T2D }\end{array}$ & SLC3OA8 \\
\hline HbA1c & rs11557154 & DCAF12 & p.R113Q & $\mathrm{T} / \mathrm{C}$ & 0.138 & $-0.009(0.002)$ & $1.70 \times 10^{-7}$ & 144045 & Novel & \\
\hline FG & rs17853166 & IKBKAP & p.S251G & $\mathrm{C} / \mathrm{T}$ & 0.026 & $-0.037(0.006)$ & $4.82 \times 10^{-11}$ & 129640 & FG & IKBKAP \\
\hline $\mathrm{HbA1c}$ & rs60980157 ${ }^{2}$ & GPSM1 & p.S391L & $\mathrm{T} / \mathrm{C}$ & 0.246 & $-0.013(0.002)$ & $6.71 \times 10^{-17}$ & 118824 & FG, T2D & GPSM1 \\
\hline
\end{tabular}




\begin{tabular}{|c|c|c|c|c|c|c|c|c|c|c|}
\hline FG & $\mathrm{rs} 60980157^{2}$ & GPSM1 & p.S391L & $\mathrm{T} / \mathrm{C}$ & 0.254 & $-0.014(0.002)$ & $2.35 \times 10^{-9}$ & 110915 & $\mathrm{FG}, \mathrm{T} 2 \mathrm{D}$ & GPSM1 \\
\hline \multicolumn{11}{|c|}{ 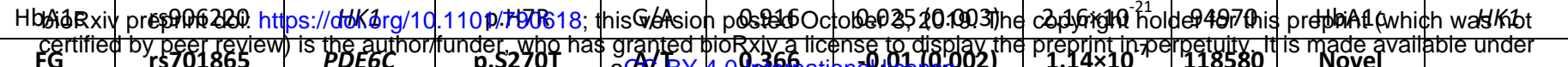 } \\
\hline HbA1c & rs61732434 & OR51V1 & p.S161N & T/C & 0.008 & $-0.052(0.009)$ & $1.75 \times 10^{-8}$ & 127507 & Novel & \\
\hline HbA1c & rs415895 & SWAP70 & p.Q447E & $G / C$ & 0.641 & $-0.013(0.001)$ & $1.15 \times 10^{-21}$ & 138028 & Novel & \\
\hline HbA1c & rs117706710 & AMPD3 & p.V311L & T/G & 0.009 & $0.037(0.006)$ & $2.32 \times 10^{-10}$ & 144048 & Novel & \\
\hline FG & rs2167079 & $A C P 2$ & p.R29Q & $T / C$ & 0.340 & $0.016(0.002)$ & $7.99 \times 10^{-15}$ & 129580 & FG & MADD \\
\hline HbA1c & rs35233100 & MADD & p.R766X & $T / C$ & 0.055 & $-0.015(0.003)$ & $1.13 \times 10^{-8}$ & 144034 & FG & MADD \\
\hline FG & rs35233100 & MADD & p.R766X & $T / C$ & 0.054 & $-0.029(0.004)$ & $1.46 \times 10^{-12}$ & 126231 & FG & MADD \\
\hline FG & rs $56200889^{2}$ & ARAP1 & p.Q802E & $C / G$ & 0.270 & $-0.016(0.002)$ & $1.79 \times 10^{-14}$ & 122674 & FG & ARAP1 \\
\hline HbA1c & rs643788 & DPAGT1 & p.1393V & $\mathrm{C} / \mathrm{T}$ & 0.425 & $-0.006(0.001)$ & $1.77 \times 10^{-7}$ & 144009 & Novel & \\
\hline $\mathrm{FI}^{1}$ & rs145878042 & RAPGEF3 & p.L300P & G/A & 0.011 & $-0.054(0.01)$ & $1.15 \times 10^{-7}$ & 91485 & Novel & \\
\hline HbA1c & rs2732481 & ZNF641 & p.Q363P & $\mathrm{G} / \mathrm{T}$ & 0.315 & $-0.009(0.001)$ & $2.07 \times 10^{-11}$ & 142280 & HbA1c & SENP1 \\
\hline HbA1c & rs3184504 & SH2B3 & p.W262R & $\mathrm{C} / \mathrm{T}$ & 0.567 & $0.007(0.001)$ & $5.98 \times 10^{-8}$ & 138551 & HbA1c & ATXN2 \\
\hline 2hGlu & rs $1169288^{2}$ & HNF1A & p.175L & $C / A$ & 0.345 & $0.06(0.011)$ & $7.90 \times 10^{-9}$ & 44278 & $\mathrm{~T} 2 \mathrm{D}$ & HNF1A \\
\hline HbA1c & COSM147717 & ATP11A & p.M317V & $\mathrm{G} / \mathrm{A}$ & 0.748 & $0.009(0.001)$ & $3.77 \times 10^{-12}$ & 144022 & HbA1c & $\begin{array}{l}A T P 11 A, T U B \\
\quad G C P 3\end{array}$ \\
\hline HbA1c & rs229587 & SPTB & p.S439N & $T / C$ & 0.357 & $0.007(0.001)$ & $2.60 \times 10^{-8}$ & 134780 & Novel & \\
\hline HbA1c & rs35097172 & SLC25A47 & $\begin{array}{c}\text { splice region } \\
\text { variant, 5' } \\
\text { UTR variant }\end{array}$ & $\mathrm{T} / \mathrm{C}$ & 0.216 & $-0.008(0.002)$ & $5.67 \times 10^{-8}$ & 144028 & FG & SLC25A47 \\
\hline 2hGlu & rs3784634 & VPS13C & p.R974K & $\mathrm{T} / \mathrm{C}$ & 0.540 & $-0.069(0.011)$ & $6.40 \times 10^{-10}$ & 37217 & 2hGlu & $\begin{array}{l}\text { VPS13C/ } \\
\text { C2CD4A/ } \\
\text { C2CD4B }\end{array}$ \\
\hline $\mathrm{HbA1}^{1}$ & rs3747481 & PRR14 & p.P359L & $T / C$ & 0.261 & $0.009(0.002)$ & $3.30 \times 10^{-8}$ & 103338 & Novel & \\
\hline HbA1c & rs201226914 & PIEZO1 & p.L939M & $T / G$ & 0.002 & $-0.159(0.015)$ & $4.42 \times 10^{-26}$ & 144024 & HbA1c & CDT1,CYBA \\
\hline $2 \mathrm{hGlu}$ & rs72839768 ${ }^{4}$ & DVL2 & p.T529l & $\mathrm{A} / \mathrm{G}$ & 0.020 & $0.197(0.03)$ & $4.10 \times 10^{-11}$ & 57866 & $\mathrm{~T} 2 \mathrm{D}$ & $S L C 16 A 13$ \\
\hline HbA1c & rs2748427 & TMC6 & p.W125R & $\mathrm{G} / \mathrm{A}$ & 0.233 & $0.027(0.002)$ & $8.56 \times 10^{-70}$ & 132326 & HbA1c & TMC6 \\
\hline HbA1c & rs7225887 & B3GNTL1 & p.A163T & $\mathrm{T} / \mathrm{C}$ & 0.211 & $-0.015(0.002)$ & $5.73 \times 10^{-22}$ & 125749 & HbA1c & $\begin{array}{l}\text { FN3KRP, } \\
\text { FN3K }\end{array}$ \\
\hline HbA1c & rs35413309 & RGS9BP & p.A223V & $T / C$ & 0.030 & $-0.02(0.004)$ & $1.42 \times 10^{-8}$ & 141598 & Novel & \\
\hline $2 \mathrm{hGlu}$ & rs $1800437^{2}$ & GIPR & p.E318Q & $C / G$ & 0.217 & $0.103(0.011)$ & $2.59 \times 10^{-23}$ & 56252 & $2 \mathrm{hGlu}$ & GIPR \\
\hline FG & rs $17265513^{3}$ & ZHX3 & p.N310S & $\mathrm{C} / \mathrm{T}$ & 0.188 & $0.016(0.002)$ & $2.59 \times 10^{-10}$ & 126253 & FG & $\mathrm{ZHX3}$ \\
\hline HbA1c & rs855791 & TMPRSS6 & V727A & $\mathrm{G} / \mathrm{A}$ & 0.577 & $-0.019(0.001)$ & $9.46 \times 10^{-51}$ & 143907 & $\mathrm{HbA1c}$ & TMPRSS6 \\
\hline FG & rs15943 & МАРЗК15 & p.Q1083E & C/G & 0.005 & $-0.084(0.014)$ & $2.83 \times 10^{-9}$ & 67004 & Novel & \\
\hline FG & rs56381411 & MAРЗК15 & p.G670S & $T / C$ & 0.005 & $-0.085(0.013)$ & $1.51 \times 10^{-11}$ & 62319 & Novel & \\
\hline HbA1c & rs2229241 & RENBP & $\begin{array}{c}\text { splice } \\
\text { acceptor } \\
\text { variant }\end{array}$ & $\mathrm{C} / \mathrm{T}$ & 0.012 & $-0.123(0.007)$ & $1.14 \times 10^{-62}$ & 95622 & HbA1c & G6PD \\
\hline HbA1c & rs1050828 & G6PD & p.V68M & $T / C$ & 0.007 & $-0.334(0.008)$ & $7.41 \times 10^{-322}$ & 112209 & HbA1c & G6PD \\
\hline
\end{tabular}

Table 1. Single-point coding variant associations meeting the significant threshold for coding variants of $\boldsymbol{P}<\mathbf{2 . 2} \times \mathbf{1 0}^{-\mathbf{7}}$. This table includes all novel coding variants meeting this threshold, irrespective of whether they fall in completely new loci or in previously-established loci, provided that the association at the established locus was not shown to be due to a non-coding variant (Table S3) or another coding variant at the same locus. Novel loci are highlighted in bold. HbA1c: glycated haemoglobin; FG: fasting glucose; Fl: fasting insulin; 2hGlu: $2 \mathrm{~h}$ glucose; Alleles E/O: effect allele/other allele; Freq. Effect Allele: frequency of effect allele; Effect (SE): effect size (standard error); $P$ : $\mathrm{p}$-value; $\mathrm{N}$ : number of samples in the analysis; Novel/previous glycemic trait association: Novel corresponds to a new association result; Locus name of previous association - name used for previously-reported locus. ${ }^{1}$ Significant in the European-only analysis in our study. ${ }^{2}$ Genome-wide significant association with T2D since date of analysis (Mahajan et al., 2018b). ${ }^{3}$ Association with T2D at $P<1 \times 10^{-4}$ since date of analysis (Mahajan et al., 2018b). ${ }^{4}$ T2D locus identified in Japanese (Hara et al.,2014) and Mexican (Williams et al., 2014) populations only. The date of our exomes analysis is May 2015. Related to Table S3. 
bioRxiv preprint doi: https://doi.org/10.1101/790618; this version posted October 3, 2019. The copyright holder for this preprint (which was not certified by peer review) is the author/funder, who has granted bioRxiv a license to display the preprint in perpetuity. It is made available unde

\begin{tabular}{|c|c|c|c|c|c|c|c|}
\hline \multirow[b]{2}{*}{ Trait } & \multirow[b]{2}{*}{ Gene } & \multicolumn{4}{|c|}{$\begin{array}{l}\text { NSbroad mask } \\
\text { NSternational ticense. }\end{array}$} & \multicolumn{2}{|c|}{ NSstrict mask } \\
\hline & & N var & $\boldsymbol{P}_{\text {burden }}$ & $P_{S K A T}$ & $\mathbf{N}$ var & $\boldsymbol{P}_{\text {burden }}$ & $P_{S K A T}$ \\
\hline FG & G6PC & 9 & $1.41 \times 10^{-6}$ & $1.32 \times 10^{-5}$ & 3 & $1.41 \times 10^{-3}$ & $7.43 \times 10^{-4}$ \\
\hline $\mathrm{FI}$ & G6PC & 8 & $1.62 \times 10^{-6}$ & $8.58 \times 10^{-6}$ & 3 & $1.85 \times 10^{-3}$ & $7.80 \times 10^{-3}$ \\
\hline $\mathrm{HbA1c}$ & $T F$ & 10 & $2.15 \times 10^{-6}$ & $5.98 \times 10^{-3}$ & 3 & $5.48 \times 10^{-2}$ & $5.48 \times 10^{-2}$ \\
\hline FG & МАР $3 К 15$ & 18 & $1.86 \times 10^{-25}$ & $1.07 \times 10^{-18}$ & 7 & $1.34 \times 10^{-14}$ & $4.01 \times 10^{-11}$ \\
\hline $\mathrm{HbA1c}$ & МАРЗК15 & 18 & $1.27 \times 10^{-7}$ & $1.53 \times 10^{-04}$ & 7 & $2.65 \times 10^{-4}$ & $9.46 \times 10^{-3}$ \\
\hline FG & G6PC2 & 18 & $4.09 \times 10^{-67}$ & $5.38 \times 10^{-58}$ & 7 & $7.8 \times 10^{-69}$ & $3.83 \times 10^{-56}$ \\
\hline $\mathrm{HbA1c}$ & G6PC2 & 18 & $6.18 \times 10^{-30}$ & $4.65 \times 10^{-27}$ & 7 & $1.04 \times 10^{-31}$ & $1.92 \times 10^{-26}$ \\
\hline FG & SLC30A8 & 13 & $5.69 \times 10^{-4}$ & $6.42 \times 10^{-11}$ & 7 & $6.55 \times 10^{-11}$ & $3.74 \times 10^{-10}$ \\
\hline $\mathrm{HbA1c}$ & SLC3OA8 & 12 & $7.20 \times 10^{-8}$ & $2.18 \times 10^{-5}$ & 6 & $5.66 \times 10^{-8}$ & $3.22 \times 10^{-6}$ \\
\hline FG & VPS13C & 52 & $9.66 \times 10^{-6}$ & $3.73 \times 10^{-7}$ & 26 & $1.27 \times 10^{-5}$ & $1.44 \times 10^{-5}$ \\
\hline
\end{tabular}

Table 2. Gene-based results from broad (NSbroad mask) and strict (NSstrict mask) analyses. Genes in bold are newly discovered from this effort. $\mathrm{N}$ var: total number of variants in that gene-based analysis; $P_{\text {burden: }}$ p-value from burden test which assumes all variants have the same direction of effect; $P_{\text {SKAT }}: p$-value from SKAT test which allows for different directions of effect between variants. The lowest $p$-value is highlighted in bold. Related to Table S4. 